\title{
What are you waiting for?
}

Real-time integration of cues for fricatives suggests encapsulated auditory memory.

\author{
Marcus E. Galle Jamie Klein-Packard \\ Dept. of Psychological and Brain Sciences, University of Iowa \\ Kayleen Schreiber \\ Interdisciplinary Program in Neuroscience \\ University of Iowa \\ and \\ Bob McMurray \\ Dept. of Psychological and Brain Sciences, Dept. of Communication Sciences and Disorders, \\ Dept. of Linguistics and Dept. of Otolaryngology \\ University of Iowa
}

\author{
Corresponding Author \\ Bob McMurray \\ W311 SSH \\ Dept. of Psychological and Brain Sciences \\ University of Iowa \\ Iowa City, IA 52242 \\ 319-335-2408 \\ Bob-mcmurray@uiowa.edu
}




\begin{abstract}
Speech unfolds over time and the cues for even a single phoneme are rarely available simultaneously. Consequently, to recognize a single phoneme listeners must integrate material over several hundred milliseconds. Prior work contrasts two accounts: 1) a memory buffer account in which listeners accumulate auditory information in memory and only access higher level representations (i.e. lexical representations) when sufficient information has arrived; and 2) an immediate integration strategy in which lexical representations can be partially activated on the basis of early cues and then updated when more information arises. These studies have uniformly shown evidence for immediate integration for a variety of phonetic distinctions. We attempted to extend this to fricatives, a class of speech sounds which requires not only temporal integration of asynchronous cues (the frication, followed by the formant transitions 150-350 msec later), but also integration across different frequency bands, and compensation for contextual factors like coarticulation. Experiments employed eye-movements in the visual world paradigm and showed clear evidence for a memory buffer. Results were replicated in five experiments, ruling out methodological factors and tying the release of the buffer to the onset of the vowel. These findings support a general auditory account for speech by suggesting that the acoustic nature of particular speech sounds may have large effects on how they are processed. It also has major implications for theories of auditory and speech perception by raising the possibility of an encapsulated memory buffer in early auditory processing.
\end{abstract}

Keywords: Speech Perception; Spoken Word Recognition; Cue Integration; Real time processing; fricatives; auditory memory 


\section{Introduction}

An omnipresent challenge in spoken language comprehension is time. Because speech unfolds over time, at any moment, listeners must engage memory processes of some kind to retain and integrate prior information, they must make inferences about upcoming materials, and they often must revise prior decisions. And they must accomplish these tasks rapidly, as speech unfolds at a rate of 3-5 words per second.

Consider the problem of revising a prior decision, a dominating issue in sentence processing (Frazier \& Rayner, 1982; Tanenhaus \& Trueswell, 1995). Here garden path sentences (e.g., the researchers reviewed unfairly responded forcefully) illustrate cases in which an early decision about how to interpret a word (e.g., to treat reviewed as a main clause verb) must be revised later (e.g., at unfairly it must be a reduced relative). Such effects arise in every level of language. For example, in speech when an initial phoneme of a word is mistaken, the ambiguity may not be resolved until later (McMurray, Tanenhaus, \& Aslin, 2009). Such a revision process would seem to require some form of memory, to maintain lower level information (e.g., the sequences of words, phonemes) to be later reinterpreted (Just \& Carpenter, 1992). However, interactive activation models can perform such revision without explicit memory buffers (MacDonald, Pearlmutter, \& Seidenberg, 1994; McClelland \& Elman, 1986; McRae, Spivey-Knowlton, \& Tanenhaus, 1998; Norris \& McQueen, 2008) (and see Bharucha, 1987, for parallel issues in another temporally organized domain, music). In these models, multiple interpretations (e.g., words, syntactic parses) are maintained in parallel. The degree to which activation is maintained for less favored hypotheses serves as a sort of memory, allowing these hypotheses to be reactivated quickly in the face of new evidence.

A related problem created by the temporal unfolding input is that of integrating 
asynchronous information. For most decisions (phonological, lexical, syntactic), at any given moment there is rarely sufficient information to make an unambiguous decision. At face value this also seems to require explicit memory to maintain earlier information until a decision can be made. However, interactive models can accomplish the same goal implicitly: as each "unit" of information arrives activation for hypotheses are updated and the maintenance of this graded activation then serves as an implicit memory for the strength of the evidence.

As these examples highlight, while memory is clearly required for dealing with time, it is not clear whether it is handled implicitly (in terms of activation dynamics) or with an explicit store. Ample evidence exists for links between working memory and language processing, particularly in sentence processing (Caplan \& Waters, 1999; Fedorenko, Gibson, \& Rohde, 2006; Gathercole \& Baddeley, 1990; Just \& Carpenter, 1992; King \& Just, 1991), but potentially in word recognition as well (Zhang \& Samuel, 2018). However, such effects can also be explained by computational accounts in which multiple levels of analysis are computed simultaneously in recurrent distributed representations (Christiansen \& MacDonald, 2009; Gupta \& Tisdale, 2009) (and seeElman, 1990, 2009; Gaskell \& Marslen-Wilson, 1997, for broader models of this type).

A more unambiguous source of evidence for a memory based approach to integration over time could come from work investigating continuous cascades among levels of processing. Modular accounts of language processing (e.g., Frazier, 1987), or strong memory accounts, predict that processing at one level (e.g., syntactic) should be complete before processing at the next level (semantic) begins. In contrast, a continuous cascade argues that intermediate, incomplete processing states can be passed on to the next level of processing before they have been fully resolved. If evidence against such a cascade is seen, it would suggest the necessity of some lower-level encapsulated memory process. 
At this point, work on every level of language offers evidence for continuous cascades. Phonetic cues processing cascades to phonological and lexical processing (Andruski, Blumstein, \& Burton, 1994; McMurray et al., 2009), phonological processing cascades to affect lexical processing (Marslen-Wilson \& Zwitserlood, 1989), word-form recognition cascades to semantic processing (Apfelbaum, Blumstein, \& McMurray, 2011; van den Brink, Brown, \& Hagoort, 2006; Zwitserlood, 1989), and syntactic processing (Levy, Bicknell, Slattery, \& Rayner, 2009). This stands as strong evidence for interactive over modular accounts.

The present study originally sought to build on this work in the context of integrating asynchronous cues to phonemes. For example, Voice Onset Time (VOT, the dominant cue for distinguishing /b/ from /p/) precedes Vowel Length (VL, a secondary cue, described in more detail shortly). Prior work on this has shown clear evidence of continuous cascades (McMurray, Clayards, Tanenhaus, \& Aslin, 2008; Toscano \& McMurray, 2012, 2015): lexical level processing is sensitive to VOT from the earliest moments, and is later updated by VL (see also, Kingston, Levy, Rysling, \& Staub, 2016; Mitterer \& Reinisch, 2013; Reinisch \& Sjerps, 2013). In contrast, a memory-buffer account predicts that lexical activation can only be initiated once both cues arrive, in which case their effects should be simultaneous and late. This continuous cascade is consistent with accounts - like interactive activation accounts - which do not require explicit memory buffers: partial activation for phonological or lexical units preserves the strength of early evidence.

We sought to characterize temporal integration of acoustic cues in sibilant fricatives (/s/ and $/ \mathrm{J} /$. We examined these because the two major perceptual cues - the frication-spectrum and the formant transitions in the vocoid - are highly acoustically distinct from each other. We expected listeners to show the same immediate integration strategy for fricatives as for other sounds. However, we unexpectedly found that listeners used a memory buffer strategy. Across six 
experiments, listeners waited for both the frication-spectrum and formant transitions to arrive before activating lexical candidates.

This offers unambiguous evidence for at least one component of sublexical processing that is encapsulated from higher level lexical processing. This has not been observed before. Virtually all prior research supports a continuous cascade between so-called speech perception processes (e.g., cue-integration, categorization and talker compensation) and lexical access (Andruski et al., 1994; Goldinger, 1998; McMurray, Clayards, et al., 2008; McMurray, Tanenhaus, \& Aslin, 2002; Reinisch \& Sjerps, 2013). In these cases, preliminary stages of perceptual processing impact lexical activation before they are completed. As we described, analogous continuous cascades have been observed in higher level language (Levy et al., 2009; van den Brink et al., 2006), and debates around the necessity of explicit memory processes are ongoing (Christiansen \& MacDonald, 2009). In contrast, the present study offers evidence for at least one perceptual process that does not cascade to lexical processing, and clear evidence for some form of explicit, encapsulated memory process. Thus, these results have implications beyond the narrow issues of fricative perception.

We next review work on temporal integration in word recognition and speech perception. We then present six experiments that offer clear evidence for a memory-buffer strategy and rule out several alternative accounts.

\section{Spoken Word Recognition and Speech Perception}

Spoken word recognition is a canonical problem of temporal integration - listeners must assemble words across several phonemes and it is not until often late in the word that the word can be identified. There is clear evidence that listeners do not wait to begin making lexical 
decisions. Measures like gated stimuli, cross-modal priming and eye-tracking show that at early points multiple lexical entries are active in parallel (Allopenna, Magnuson, \& Tanenhaus, 1998; Marslen-Wilson \& Tyler, 1980; Marslen-Wilson \& Zwitserlood, 1989; Tyler \& Wessels, 1985). These partially active candidates in turn activate their semantic meanings and syntactic parses even before a single word is resolved (Apfelbaum et al., 2011; Gaskell \& Marslen-Wilson, 1997; Levy et al., 2009; Marslen-Wilson, 1987; Marslen-Wilson \& Tyler, 1980; Yee \& Sedivy, 2006; Zwitserlood, 1989). This integration can thus be accomplished implicitly, as simply the accumulation of evidence across words (McClelland \& Elman, 1986; Norris \& McQueen, 2008) without the need for dedicated memory.

While the case for immediate activation is well-accepted in spoken word recognition (Dahan \& Magnuson, 2006; Weber \& Scharenborg, 2012), there is less work in speech. In speech the rapid arrival of information and the variability of individual cues raises the possibility that buffered processing may be helpful. Phonemes leave cues scattered over several hundred milliseconds. For example, vowels are cued by formants within the target vowel (Peterson \& Barney, 1952), its length (Hillenbrand, Clark, \& Houde, 2000) and by formants in subsequent and earlier vowels (Beddor, Harnsberger, \& Lindemann, 2002; Cole, Linebaugh, Munson, \& McMurray, 2010). Similarly, voicing (e.g., /b/vs /p/, /d/ vs /t/) is canonically cued by Voice Onset Time (VOT: the time lag between the release of the articulators and the onset of voicing) (Lisker \& Abramson, 1964). Here, VOT can be identified at word onset and is considered the primary cue. VOTs near $0 \mathrm{msec}$ are usually heard as /b/ and long VOTs (50-60 msec) as /p/. However, as a temporal cue, VOT changes with speaking rate. Consequently, the length of the vowel (VL) is a useful secondary cue, with long VLs leading to more /b/ decisions, and short VLs to more /p/ decisions (Joanne L. Miller \& Volaitis, 1989; Summerfield, 1981). Critically, 
VL cannot be identified until several hundred msec after VOT.

In this context, there are multiple possibilities for listeners could integrate such asynchronous cues. Under a memory buffer approach VOT is stored in memory, and VL is added to this buffer when it can be identified later in the word. Information in this buffer is encapsulated from other aspects of language processing (until both are available). As a result, no decision about the phoneme can be made until both cues are identified. It is rarely the case that one cue is sufficient for phoneme perception (Lisker, 1986; McMurray \& Jongman, 2011). As a result, this strategy could in principle lead to more accurate perception, particularly if early cues are unreliable or if it is difficult to overcome early, inaccurate judgements. Since phoneme decisions cascade to affect lexical access, an error in phoneme perception would lead to erroneously accessing the wrong word (and its associated meaning), digging into the wrong meaning. By delaying a phoneme decision, listeners could avoid this problem. Given the notorious variability and ambiguity in speech cues, this may heighten the need for such accuracy. A buffered strategy, however, may come at a cost. Under this view, lexical access may not be able to make early partial inferences on the basis of initial information (since this would still be in the buffer) and would have to wait to begin accessing semantic representations until sufficient information is available. This would necessarily delay processing (if early cues are sufficient).

Alternatively, under an immediate integration account, listeners make partial commitments as soon as VOT is identified. Multiple candidate phonemes (/b/ and /p/, and downstream lexical candidates) would be partially activated and later updated when VL is identified. This strategy may in principle be faster, as listeners can make at least partial commitments to some lexical items (and rule out others) before they have all of the information needed to identify the initial phoneme. However, it may less accurate (particularly early in 
processing) because partial commitments to phonemes or even words are made using insufficient information.

These hypotheses are extremes and there are intermediate possibilities. While both make similar predictions once all of the cues are available, they differ at earlier points. Immediate integration predicts listeners' phoneme and lexical decisions are biased by individual cues as soon as they arrive. That is, if two cues are asynchronous, we should see asynchronous effects of each cue on lexical activation, since words can be activated by the first cue without waiting for the second. In contrast, a memory buffer account predicts lexical access will be delayed until the second arrives, yielding synchronous effects.

\section{Immediate Integration in Speech Perception}

The first study that attempted to differentiate these strategies used a speeded response paradigm. Joanne L Miller and Dexter (1988) examined VOT and VL and found that when asked to answer quickly, listeners made voicing judgments using largely VOT; VL had almost no effect. However, responses initiated after more time showed stronger effects of VL. Because VOT arrives first, this suggests listeners can make decisions as soon as any information is available and do not have to wait for all of the information. However, this paradigm forces people to respond. This raises the possibility that people can use cues immediately when they have to, even if they do not spontaneously do so in normal listening.

More recent studies contrasted these models by taking advantage of the Visual World Paradigm (VWP; Tanenhaus, Spivey-Knowlton, Eberhard, \& Sedivy, 1995) to measure processing as it is ongoing - without forcing an early response. In the VWP, participants select a pictured referent of a spoken word while eye-movements are monitored as a measure of how 
strongly they are considering each word. Fixations unfold rapidly over the course of the trial, and reflect processing over time. Crucially, since fixations are directed to pictures, the degree to which competitors (e.g,, peach after hearing beach) are fixated more than unrelated items can reveal how strongly those words are considered at a lexical/semantic level.

These properties of the VWP afford the opportunity to test for immediate integration or buffers in speech. Since fixations to referents require accessing semantic properties, we can ask when individual cues affect lexical/semantic processing as they are heard in real-time. Here a buffer model predicts no differential fixations to one referent over another until all the cues have arrived, while immediate integration predicts immediate effects as soon as the first cue arrives.

McMurray, Clayards, et al. (2008) presented listeners with minimal pairs in which both VOT and VL were manipulated. The set of pictures included the experimental pair (e.g. beach and peach) and two fillers (e.g. rake and lake). The proportion of fixations to each was computed over time and used to estimate the bias to fixating $/ \mathrm{b} /$ or $/ \mathrm{p} /$ at each $4 \mathrm{msec}$ timeslice. Next, the effect of VOT and VL was computed by asking how strongly each cue influenced this bias over time. This showed that eye movements were affected by VOT significantly before VL, suggesting VOT was used to access lexical entries as soon as it arrived, without waiting for VL.

This result has been replicated for several other speech contrasts. The original study also showed it for integrating manner of articulation (b/w) and VL, and two studies by Toscano and McMurray $(2012,2015)$ replicated it with VOT and VL continua constructed from natural recordings and in sentence contexts. More importantly, (Reinisch \& Sjerps, 2013) examined Dutch vowels which are distinguished by length. They investigated second formant frequency (available at vowel onset) and VL (available later). In this case the major cue, vowel length, is present only after the secondary cue, F2 frequency (a reversal of prior work in which the primary 
cue comes first). They also found immediate, asynchronous effects supporting immediate integration. This extends prior work by showing that this conclusion is not limited to consonants, and that immediate integration can also be seen when the weaker cue arrives first.

Immediate integration has also been extended beyond bottom-up factors. Kingston et al. (2016) showed immediate use of lexical information (e.g., the Ganong effect), and Mitterer and Reinisch (2013) showed that retuned phoneme categories exert an immediate bias on perception. Similarly, it has long been known that word frequency affects lexical processing from the earliest moments of perception (Dahan, Magnuson, \& Tanenhaus, 2001; Dufour, Brunellière, \& Frauenfelder, 2013). This reinforces the ubiquity of this finding in speech perception.

\section{Fricatives}

Fricatives require integration of a large number of cues (Jongman, Wayland, \& Wong, 2000 ) in both the frication and vocalic (vocoid) portions. We examined fricatives for several reasons. First, the two asynchronous cues we studied (frication-spectrum and formant transition) are highly acoustically distinct from each other, appearing in entirely different frequency bands (Figure 1). Frication-spectra are generally above $2500 \mathrm{~Hz}$, while formant transitions are generally below $2500 \mathrm{~Hz}$. This raises the possibility of different integration strategies, as integration would need to span distinct frequency bands.

Second, prior work has not examined contextual factors, factors that influence categorization but do not directly cue one phoneme over another. Fricatives are influenced by contextual factors like talker and coarticulation (Jongman et al., 2000; McMurray \& Jongman, 2011) to a greater extent than VOT (though perhaps less than vowels). For example, lip rounding 
(e.g., whether the subsequent vowel is an $/ \mathrm{i} /$ or $/ \mathrm{u} /$ ) alters the spectrum of the frication at onset (Daniloff \& Moll, 1974). The presence of an /i/ or $/ \mathrm{u} /$ is not a direct cue to the fricative identity (/s/'s and $/ \mathrm{g} /$ 's are both possible before each vowel). However, knowing the vowel could help listeners interpret the frication

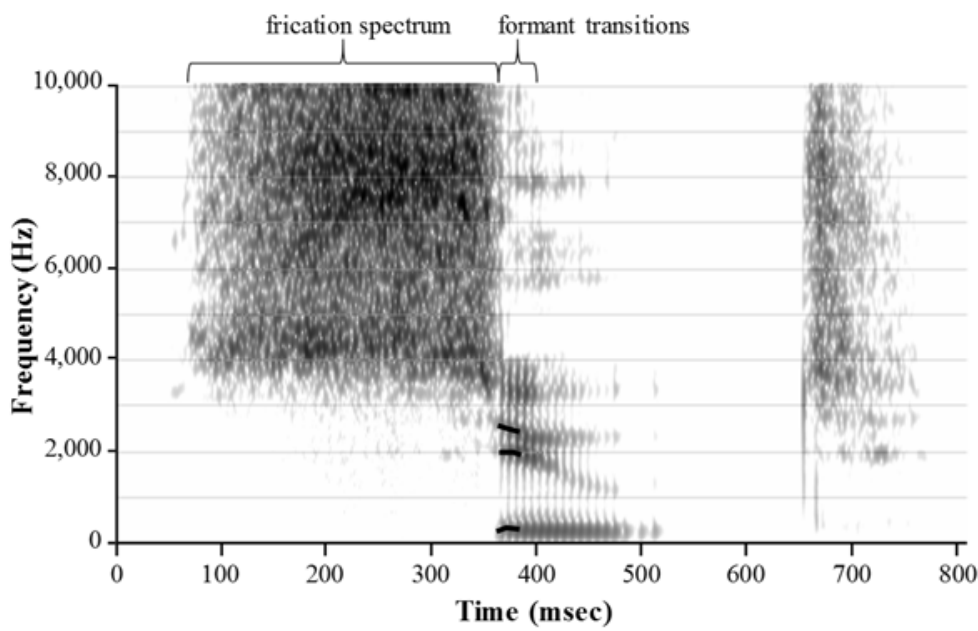

Figure 1. Spectrogram for the word suit, showing frequency differences between the frication spectrum and formant transitions (marked in black).

spectrum. If the vowel /u/ they may shift the boundary so lower frequency spectra are still treated as an /s/ (Mann \& Repp, 1980). For sounds that are strongly contextually determined, listeners may conceivably wait for the vowel before using the frication spectrum, a buffer strategy. While context dependence is also true of vowels which show immediate integration (Reinisch \& Sjerps, 2013), talker information is available throughout the vowel, obviating the need for memory. In contrast, for fricatives, talker and vowel cannot be clearly identified in the fricative and only appears at the vocoid. Thus, a buffer strategy may be needed to wait for contextual information.

We asked whether two asynchronous cues (frication spectrum and formant transition) and a contextual factor (vowel rounding) are integrated immediately. Immediate integration predicts that frication-spectrum should affect lexical competition before the later arriving formant transition and rounding. Alternatively, if listeners wait for all information prior to activating lexical entries, we should see simultaneous and late effects. With three cues, some may be integrated immediately and others via a buffer.

Despite the compelling reasons to investigate fricatives, previous research led us to 
expect largely similar results favoring immediate integration. Moreover, converging evidence from three lines of work on fricatives also hints at immediate integration. This work has not examined cue integration directly so it does not address the present question. However, it does suggest that information from sibilant fricatives can be used rapidly.

First, work with gated stimuli suggests that listeners can use small bits of frication to accurately identify fricatives (Smits, Warner, McQueen, \& Cutler, 2003; Warner, McQueen, \& Cutler, 2014). This appears to suggest that listeners do not require the vowel to begin making fricative decisions. However, even if listeners can identify the fricative without the vowel when forced to, they may still wait a more naturalistic conditions (and see Allopenna et al., 1998 for differences in the timecourse of lexical competition between gated and whole stimuli). Second, (Ishida, Samuel, \& Arai, 2016) compared fricative and stop perception with locally time reversed speech, in which short time windows are reversed to disrupt perception. They found that (in nonwords) fricatives survive longer reversal windows than stops. However, this could be consistent with either a memory buffer (that operates over a longer span for fricatives), or it could simply be a marker of the fact that fricatives contain less time varying information.

Finally, and most importantly, two VWP studies have targeted fricatives and suggest immediate utilization of frication. However, again neither directly explored the issue of cue integration. Mitterer and Reinisch (2013) used word final fricatives, and showed early identification in the eye-movements. However, they investigated the much easier $\mathrm{s} / \mathrm{f}$ contrast and word-final fricatives, where no more information would arrive after the frication (lessening the need for a buffer). More importantly, Kingston et al. (2016) showed what appears to be immediate use of frication spectra for word initial fricatives. This study, which was published after we completed data collection for this project, also examined the s/f distinction. They found 
evidence that listeners were using information in the frication at early points, though the effect size was quite small. However, unlike the studies described above, they did not manipulate a second cue to compare the timing of frication to a later cue, making it difficult to precisely compare their results to the earlier described studies. Moreover, they examined fixations to letters (not pictures) making it unclear what level of processing (lexical vs. phonemic) is being assessed. Thus, it is difficult to draw strong conclusions on immediate integration, despite some evidence for early use of the frication.

Thus, extant research potentially supports immediate integration in fricatives, but is not conclusive. In contrast, the following six studies offer strong evidence for a buffer strategy. Experiment 1 shows that frication spectrum is used simultaneously with formant transitions and lip rounding. We then rule out several methodological factors in Experiments 2-5. Finally, Experiment 6 shows definitively that the onset of the vocalic portion is what initiates the integration of all three factors.

\section{Experiment 1}

Experiment 1 asked when three sources of were used to discriminate fricatives: frication spectrum (the most important cue for place of articulation in sibilants), formant transitions (a secondary cue) and vowel rounding (a contextual factor). We assessed the timing of each factors' impact on lexical activation dynamics by monitoring their eye-movements to each (similarly to McMurray, Clayards, et al., 2008; Toscano \& McMurray, 2012).

\section{Methods}


Participants. Twenty-seven monolingual English speakers from the Iowa City area were recruited in accordance with university human subject protocols. They received $\$ 15 /$ hour or class credit for their participation. Participants reported English as their only language, normal hearing, and normal or corrected-to-normal vision.

Design. Stimuli were one-syllable English words from two contrast sets: /, $\mathrm{s} /$ and $/ \mathrm{g}, \mathrm{k} /$ (for fillers). There were four fricatives pairs with rounded vowels and four with unrounded vowels (Table 1). Table 1: Word pairs used in Experiment 1.

\begin{tabular}{|c|c|c|c|c|}
\hline Vowel & \multicolumn{2}{|c|}{ Fricative Pairs } & \multicolumn{2}{c|}{ Stop-Voicing Pairs } \\
\hline \multirow{4}{*}{ Unrounded } & Seep & Sheep & Kale & Gale \\
\cline { 2 - 5 } & Seat & Sheet & Card & Guard \\
\cline { 2 - 5 } & Same & Shame & Cage & Gauge \\
\cline { 2 - 5 } & Save & Shave & Cap & Gap \\
\hline \multirow{4}{*}{ Rounded } & Sew & Show & Coat & Goat \\
\cline { 2 - 5 } & Sore & Shore & Coop & Goop \\
\cline { 2 - 5 } & Sue & Shoe & Cool & Ghoul \\
\cline { 2 - 5 } & Suit & Shoot & Coal & Goal \\
\hline
\end{tabular}

There were not sufficient picturable minimal pairs using the exact same vowel, so rounded pairs included both $/ \mathrm{ov} /$ and /u/, while unrounded pairs included /i/ and /I/.

For fricative pairs, a 6 step continuum for frication spectrum was created. To manipulate formant transitions, these were spliced onto vocoids taken from /s/- and / $/$ /-initial words. This led to 4 pairs $\times 2$ vowel rounding $\times 6$ frication steps $\times 2$ formant transitions or 96 total test stimuli (Table 1). Each stimulus was heard on six trials, for 576 test trials. Filler stimuli were $8 \mathrm{~g} / \mathrm{k}$ minimal pairs which matched the fricative pairs on vowel rounding (Table 1). Filler stimuli were not manipulated. Each was presented 36 times, for 576 filler trials. The 1152 total trials were split between two one hour test sessions, spaced at least a week apart.

Auditory Stimuli. Frication spectra continua were constructed from natural speech using custom tools we developed to create well-controlled, natural sounding fricative stimuli (Galle, 2014; McMurray, Farris-Trimble, Seedorff, \& Rigler, 2016). The primary cue to fricative place of articulation in sibilants is the spectral mean of the frication or the location of the peak frequency (Forrest, Weismer, Milenkovic, \& Dougall, 1988; Jongman et al., 2000). Ideally, a 
continuum should have a single peak which shifts from around $6500 \mathrm{~Hz}$ for an /s/ to around 4000 $\mathrm{Hz}$ for an $/ \mathrm{J} /$. The most common method of generating fricative continua is intensity mixing or sample averaging in which the waveforms of the $/ \mathrm{s} /$ and $/ \mathrm{g} /$ are averaged in various increments. However, an intermediate step of a continuum created in this way contains two peaks (one at 6500 , and one at 4000) each at half intensity, rather than a single peak at $5250 \mathrm{~Hz}$. Thus, the intensity mixing method produces a somewhat unnatural variation. While prior work using phoneme decision has not found strong differences between sample-averaging and other methods of continuum generation (Repp, 1981), we were concerned that such difference may matter for our more sensitive measure of cue integration. We overcame this using a technique introduced by McMurray et al. (2016) which estimates the spectra of each endpoint, shifts it in frequency space to create intermediate steps, and uses these to generate new fricatives. The resulting fricatives more precisely map onto a shift in the peak frequency. Matlab code is available at https://osf.io/ut9wz/.

Our process (Supplement S1 for a visualization) began with recordings of naturally produced fricatives. We recorded 5-10 utterances of the $/ \mathrm{J} /$ - and $/ \mathrm{s} /$-initial word. These were produced by a male talker in a soundproof booth and digitized with a Kay CSL 4300B. We then chose the five best exemplars of each word. Frication portions were excised at zero crossing closest to the onset of voicing. We next extracted the long-term average spectrum from each exemplar. This spectrum was translated in frequency space so that all of the spectra (for both /s/ and $/ \mathrm{J} /$ ) had the same peak frequency, centered at the average peak across all the tokens. After spectral alignment, the amplitudes were averaged across spectra from the same fricative to create a prototypical spectra of each endpoint (e.g. one / $/$ / spectra and one /s/ spectra), but still aligned at the center frequency. This shifting onto a common peak frequency thus preserves the shape 
around the peak, while the averaging across multiple tokens smooths out differences elsewhere.

After creating these prototypical/s/ and / $/$ spectra, we created 6-step continua. To do this, we calculated intermediate spectral shapes by computing a weighted average of the endpoint spectra (still aligned to the average peak frequency), with the weighting ranging from $100 \% / \mathrm{s} /$ to $0 \% / \mathrm{s} /$ in six steps. Spectra were then shifted (in frequency space) to the appropriate peak frequency, with peaks linearly spaced between that of the natural /s/ and that of the natural $/ \mathrm{g} /$. Next, we used each spectra to filter white noise to create frication at each step. Finally, we extracted the envelope of all 80 recordings of the fricatives and averaged them across both /s/ and $/ / /$ tokens to create a neutral envelope. The filtered noise was multiplied by this average envelope to create the final fricatives. The average length of the fricatives was $350 \mathrm{msec}$.

We constructed prototypical fricative spectra (averaged across all words) instead of constructing a separate continuum for each word pair because we were concerned that coarticulatory differences in the spectra would provide an early cue to vowel rounding. We wanted to eliminate this (which could lead to differences in early perception as the vowel could be identified from this coarticulation). Thus, this procedure created frication in which cues for vowel rounding (in the frication) have neutral values that are not consistent with either a rounded or unrounded vowel, and listeners must wait for the vocoid to know the vowel.

We also point out that this style of continuum generation is grounded in real prototypical productions - step 1 is based on the long-term average spectrum of an actual $/ / /$ and step 6 on an actual /s/, with even (frequency) spacing between them. Thus, we did not attempt to find a particular sub-range centered on the boundary; rather we sought to capture the entire (prototypeto-prototype) range, much as we did for the other cues by using naturally produced vocoids. Each fricative was spliced onto naturally produced word endings (vocoids). Vocoids 
came from both the $/ \mathrm{s} /$ and $/ \mathrm{J} /$ endpoint of each of the 8 word-pairs, so that there were two classes of formant transitions (appropriate for an $/ \mathrm{s} /$ and $/ \mathrm{f} /$ ). Frications and voicoids overlapped by 20 msec using amplitude ramping to create a transition period. One hundred msec of silence was added to the beginning and $50 \mathrm{msec}$ of silence added to the end of each auditory stimulus.

Note that our manipulation of the formant transition is indirect. That is, the s-biased transitions of the sheep/seep are not acoustically identical to those of the shoot/suit continua (nor could they be). Thus, we refer to them as s-consistent or s-biased transitions, and our analyses aggregate over individual cue values to look at the effect of this bias overall. Similarly, our manipulation of rounding should not be seen as a manipulation of an acoustic cue at all, but rather as a manipulation of the listener's percept of the vowel as rounded or unrounded.

Although stimuli produced via this technique are partially synthesized, they sound remarkably natural and are difficult to differentiate from naturally produced fricatives. We verified this by asking participants to choose whether stimuli produced via sample averaging or this spectral shifting method sounded more natural. Despite the fact that the spectral shifting method "synthesized" fricatives from scratch, participants labeled tokens made with it as more natural sounding on $61 \%$ of the trials.

Filler stimuli were created by recording natural utterances of stop-consonant minimal pairs, from the same talker on which the fricative stimuli were based.

Visual Stimuli. Visual stimuli consisted of clipart-style images corresponding to the referent of each words, constructed using a standard lab protocol (McMurray, Samelson, Lee, \& Tomblin, 2010). For each item, several pictures were downloaded from a commercial clipart database. A focus group of graduate and undergraduate students reviewed each set of pictures and selected the clearest and most canonical exemplar for each word. These were then edited to 
obtain consistent levels of color and brightness, to eliminate distracting elements, and to make other minor modifications to ensure that each image was a highly prototypical representation of its intended word. Next, all pictures were scaled to $200 \times 200$ pixels. Finally, each picture was approved by an independent member of the research team with extensive experience with the VWP (and who was not involved in the focus groups).

Procedure. The experiment was run using Experiment Builder (SR Research, Oakville, ON, Canada). After undergoing informed consent, participants were seated in front of a PC with a 19-in. CRT monitor in a sound attenuated, dimly lit room. The eye-tracker was calibrated using a standard nine-point calibration. Auditory stimuli were presented binaurally over Sennheiser HD 280 Pro headphones. Throughout the experiment, participants were able to adjust the volume on the headphones to a comfortable level using a Samson C-que 8 amplifier.

During the first phase of each experiment, participants were familiarized to the pictures. Each picture was shown with its name printed below it. The participant studied each picture and used the spacebar to advance through the pictures at his or her own pace. After familiarization, the participant received written instructions for the experimental phase.

During the experimental phase, on each trial participants saw a display with four pictures, arrayed in a square pattern with one picture near each corner of the screen. On any given trial, two members of a fricative pair were present along with two members of a stop-consonant pair. The same voicing pairs were always paired with a given fricative pair, and this was randomly selected for each participant (McMurray et al., 2002). Pictures were $200 \times 200$ pixels (approximately $6.4^{\circ}$ at $50 \mathrm{~cm}$ ), and separated by 780 pixels $\left(24.5^{\circ}\right)$ horizontally and 524 pixels $\left(16.6^{\circ}\right)$ vertically in the display. The location of each picture was random on each trial.

At the beginning of the trial, a blue dot (50 pixels diameter) was displayed in the center 
of the screen along with the pictures. After $500 \mathrm{msec}$, the dot changed to red, indicating that the participants could click on it to hear the auditory stimulus. This allowed a short pre-scan period so the participant knew where the objects would be prior to stimulus onset (minimizing the need for eye-movements due to visual search). It also ensured that the mouse (and likely the point of gaze) was at the center of the screen at trial onset trial.

After hearing the stimulus, participants clicked the corresponding picture with an ordinary computer mouse. As in prior studies of cue integration (McMurray, Clayards, et al., 2008), there was no time limit, and participants were encouraged to take their time and perform accurately. After a picture was selected, the display disappeared and was replaced by a blank (white) screen for $500 \mathrm{msec}$ before the next trial began.

\section{Results}

Our primary analyses asked when each factor affected the fixation record. Before presenting that analysis, we start by evaluating the mouse click results (analogous to traditional phoneme identification) to document that each stimulus factor affected the explicit decision in the predicted ways. We next evaluate the fixation record as a whole to document that each factor affected fixations. Finally, we present analyses asking when these effects occurred.

Identification (Mouse-click responses). Figure 2 shows mouse clicks as a function of continuum step, separated by frication step (panel A), formant transition (panel B) and vowel rounding (panel C). Participants reliably labeled tokens at Step 1 as $/ \mathrm{J} /$ and Step 6 as /s/, regardless of either transition or vowel rounding. At points where the frication step was somewhat ambiguous, both factors influenced identification. As expected, /s/-consistent formant transitions led to more /s/ responses and / $/$ /-transitions led to more / $/$ / responses (Figure 2A); 
similarly, listeners were more

likely to respond /s/ for fricatives

preceding a rounded $(/ \mathrm{u} /$ or $/ \mathrm{ov} /)$

than unrounded $(/ \mathrm{i} /$ or $/ \mathrm{I} /)$ vowel

(Figure 2B).

To assess these patterns

statistically, we used a logistic
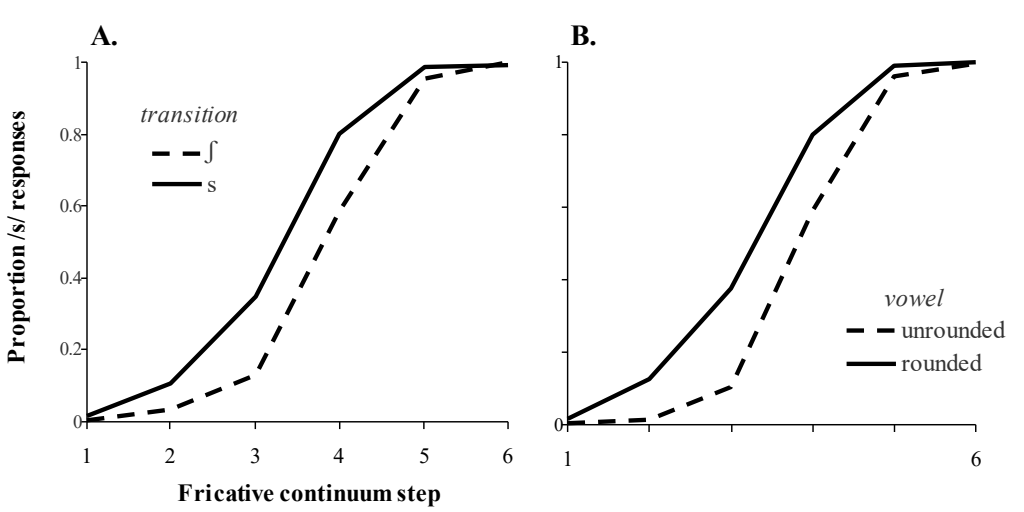

Figure 2. Proportion $/ s /$ responses as a function of frication step and (A) formant transition or (B) vowel rounding for Experiment 1. mixed effects model implemented with the lme4 package (Version 1.1-12) (Bates \& Sarkar, 2011) in $\mathrm{R}$ (Version 3.4.2). The dependent variable was binary (/s/response=1). Fixed effects included frication step (centered), transition $(/ \mathrm{s} /$ transitions $=-0.5, / \mathrm{g} /$ transitions $=+0.5)$, vowel rounding (rounded $=-0.5$; unrounded $=+0.5$ ), and their interactions. We identified the random effects structure using a forward model selection procedure (Matuschek, Kliegl, Vasishth, Baayen, \& Bates, 2017). We began with a base model that included all of the fixed effects and random intercepts for subject and word pair. Adding random slope of frication on subject significantly increased fit $\left(\chi_{2}(2)=186.8, \mathrm{p}<.001\right)$ However, more complex models failed to converge. Thus, the final model used frication, transition, rounding and their interactions as fixed effects, with random slope of frication on subject and random intercepts for word pair (1). ${ }^{1}$

$$
\begin{gathered}
\text { Response } \sim \text { Step * Transition * Rounding }+(1 \mid \text { Subject })+(\text { Step } \mid \text { Subject }) \\
+(1 \mid \text { Word pair })
\end{gathered}
$$

\footnotetext{
${ }^{1}$ We dropped covariance terms (rather than dropping random slopes) to achieve model convergence as this technique is supported by both Barr and our own Monte Carlo simulations (Seedorff \& McMurray, in preparation) as a more conservative approach.
} 
Results are in Table

2. We found a significant

main effect of frication

$(\mathrm{p}<.001)$, with increasing

likelihood of an /s/ response

at higher steps. Similarly,
Table 2. Results of a logistic mixed model examining mouse-click results for fricative stimuli in Experiment 1. $p>.1$ not shown.

there was a main effect of transition $(\mathrm{p}<.001)$ with more $/ \mathrm{s} /$ responses for $/ \mathrm{s} /$ transitions. There was also a main effect of rounding $(\mathrm{p}<.001)$ with more $/ \mathrm{s} /$ responses after rounded vowels. There was also a significant rounding $\times$ frication interaction $(\mathrm{p}<.001)$, indicating the identification slopes was shallower for /s/-transitions. Finally, rounding interacted with transition $(\mathrm{p}<.001)$ as there were more /s/ responses for rounded vowels with /s/-transitions. These results validate our stimuli by showing that listeners' overt responses match the expected pattern for these cues.

Evidence of effects in eye-movement data. We next validated that each independent variable affected the fixations as a whole. As in prior studies (McMurray, Clayards, et al., 2008; Toscano \& McMurray, 2012, 2015), the primary dependent variable was biass-f, the proportion of fixations to the /s/ object minus the proportion to the $/ \mathrm{J} /$ object. Biass- $/$ should be near 1.0 if the participant only fixated /s/, -1.0 if they only fixatied $/ \mathrm{J} /$, and near 0 if they were equibiased (or
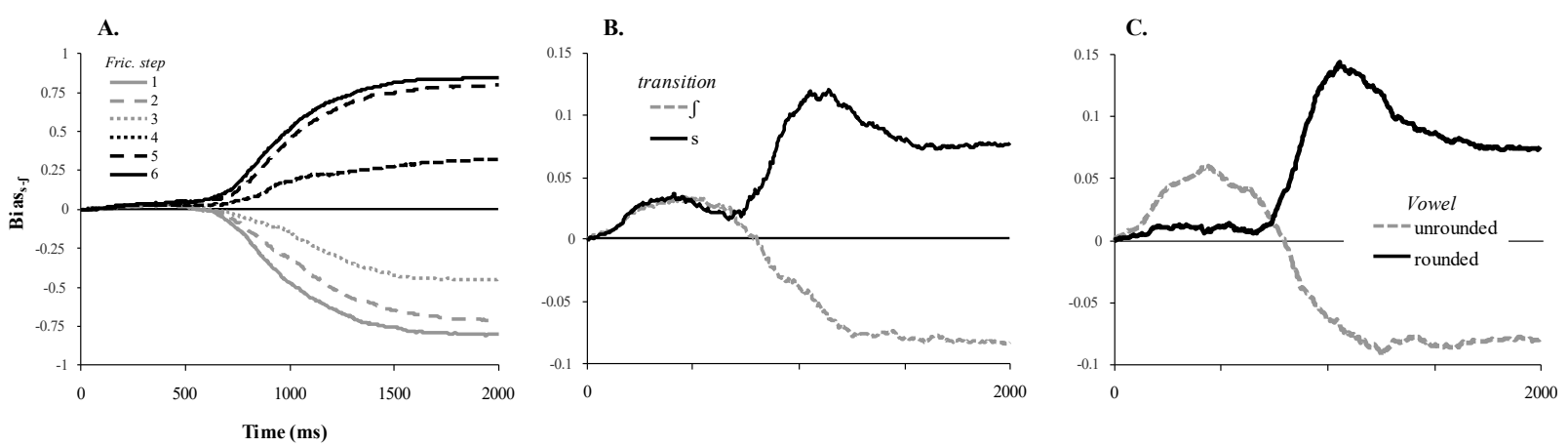

Figure 3. Bias s-fover time as a function of (A) frication step, (B) formant transition, and (C) rounding for Experiment 1. 
not looking at anything). Biass-f was computed every 4 msec. Figure 3 shows biass-fover time as a function of frication spectrum (Panel A), transition (Panel B) and rounding (Panel C).

To analyze these statistically, we computed the average biass-f between 600 and 1600 msec, as it was in this time window that bias departed from 0 . This was analyzed in a linear mixed-effects model. Fixed effects included frication step (centered); transition $(/ \mathrm{s} /$ transitions $=$ $-0.5, / \mathrm{J} /$ transitions $=+0.5)$; and vowel rounding $($ rounded $=-0.5 ;$ unrounded $=+0.5)$. The base model included all of the fixed effects and random intercepts for subject and word pair. Adding a random slope of frication on subject significantly increased fit $\left(\chi_{2}(2)=457.73, p<.001\right)$, as did adding random slopes of frication on word pair $\left(\chi_{2}(2)=97.59, \mathrm{p}<.001\right)$, transition on subject $\left(\chi_{2}(7)=16.28, p=0.0227\right)$, and transition on word pair $\left(\chi_{2}(7)=76.91, p<.001\right)$, More complex models failed to converge, so the final model used frication, transition and rounding as fixed effects, with random slopes of frication and transition on subject and frication on word-pair (2).

$$
\begin{aligned}
\text { Bias } \sim \text { Step } & * \text { Transition } * \text { Rounding }+(\text { Step } * \text { Transition } \mid \text { Subject }) \\
& +(\text { Step } \mid \text { Word pair })
\end{aligned}
$$

The complete analysis is shown in Table 3. We found a significant main effect of frication step $(\mathrm{p}<.001)$, with a stronger bias toward $/ \mathrm{s} /$ at higher fsteps. There was also an effect of transition $(\mathrm{p}=0.02)$, with more /s/-bias with /s/-consistent transitions. Finally, there was a main effect of rounding $(p=0.004)$ Table 3. Evidence of effects in eye movement data for Experiment 1. with more bias toward /s/ with a rounded than an unrounded vowel. We also found a significant frication step $\times$ p>. 1 not shown.

\begin{tabular}{lrrrrr} 
Factor & $\mathbf{B}$ & $\mathbf{S E}$ & $\mathbf{d f}$ & $\mathbf{t}$ & $\mathbf{p}$ \\
\hline Frication & 0.23 & 0.02 & 22.21 & 12.58 & $<0.001$ \\
Transition & 0.12 & 0.04 & 6.35 & 3.07 & 0.02 \\
Rounding & 0.14 & 0.03 & 6.02 & 4.59 & 0.004 \\
Frication $\times$ transition & -0.02 & 0.00 & 8.38 & 2.47 & 0.04 \\
Frication $\times$ rounding & -0.03 & 0.02 & 6.00 & 1.19 & \\
Transition $\times$ rounding & 0.09 & 0.08 & 6.00 & 1.50 & \\
Frication $\times$ trans $\times$ round & -0.05 & 0.01 & 7.09 & 3.73 & 0.007
\end{tabular}


transition interaction $(p=0.04)$. This was driven by the fact that the effect of formant transition was stronger at intermediate steps than at the endpoints. Finally, there was a significant frication $\times$ rounding $\times$ transition interaction $(\mathrm{p}=0.007)$, as the continua with both $/ \mathrm{s} /$-transitions and rounded vowels showed less effect of frication than other continua.

Timing of effects in eye-movement data. Finally, the primary analysis asked when each cue affected lexical activation. The onset of effects was estimated using a technique similar to McMurray, Clayards, et al. (2008) and originally proposed for ERPs by J. O. Miller, Patterson, and Ulrich (1998). We started by computing a measure of the effects of frication, transition and rounding on biass-fat each time step (Figure 4A). The frication effect was
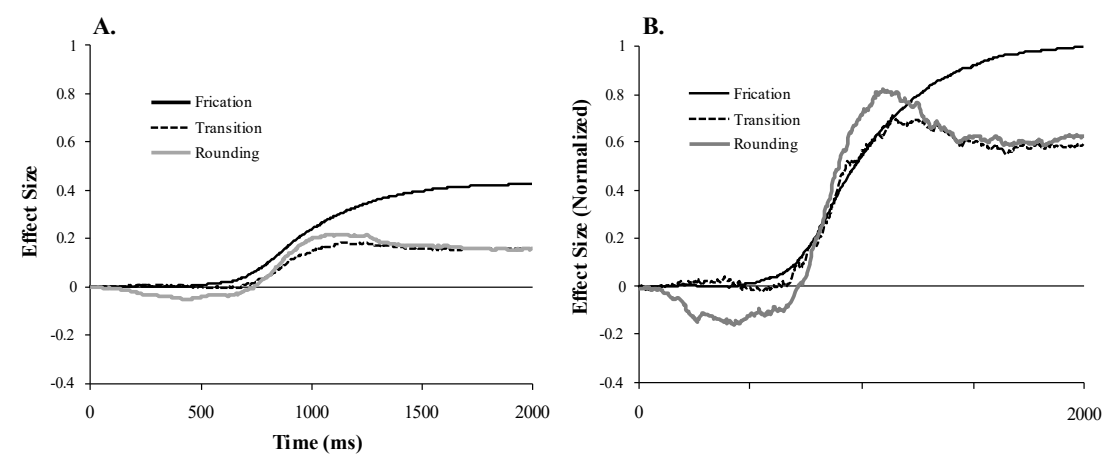

computed as the slope of a linear regression relating biass-jto Figure 4. (A) Effect size as a function of time for frication, transition, and rounding for Experiment 1 (B) Normalized and jackknifed effect sizes.

frication step; the transition effect was the difference in biass-fbetween the two formant transitions; and the rounding effect was the difference between rounded and unrounded vowels.

Fricative spectrum is the most robust cue to sibilant place of articulation (Forrest et al., 1988; Jongman et al., 2000), and is more heavily weighted by listeners (Figure 2). Thus, it had larger effects on biass-f than transition or rounding (Figure 4A). Since our primary questions concerned the timing (not the strength) of each effect, we next equated measures for absolute magnitude, by dividing the effect size at each time point by the maximum of each effect across the whole timecourse for each subject (Figure 4B).

Finally, we determined the onset of each effect. We calculated when the timecourse of 
Table 4. Timing of fricative, transition, and rounding effects in eye-movement data for Experiment 1. $p>.1$ not shown.

\begin{tabular}{ccccccrrr} 
& \multicolumn{2}{c}{} & \multicolumn{2}{c}{ Fricative vs. } & \multicolumn{2}{c}{ Fricative vs. } \\
Thounding \\
Threshold & Fricative & Transition & Rounding & t(26) & p & \multicolumn{2}{c}{ t(26) } & p \\
\hline $20 \%$ & 791 & 804 & 805 & 0.74 & & 0.67 & \\
$30 \%$ & 849 & 834 & 830 & 1.03 & & 1.12 & \\
$40 \%$ & 902 & 863 & 853 & 2.90 & .007 & 2.49 & .020 \\
$50 \%$ & 964 & 889 & 872 & 3.22 & .003 & 4.99 & $<.001$
\end{tabular}

each effect crossed a fixed threshold (e.g., $20 \%$ of maximum) and remained above that threshold for at least 30 msec. We used a range of thresholds (based on McMurray, Clayards, et al., 2008; Sjerps, McQueen, \& Mitterer, 2013; Toscano \& McMurray, 2012) to ensure that results were robust. Because of noise in the timecourse of these effects for individual participants, the data were jackknifed prior to estimating effect onsets. For this, effect sizes were averaged across all subjects but one, onsets were estimated, and this process was repeated until each subject had been excluded from one averaged dataset. These effect onsets were compared across cues with paired t-tests in which the t-statistic was adjusted to reflect the fact that each jackknifed participant represents N-1 participants.

As seen in Table 4, at the $20 \%$ and $30 \%$ thresholds, the frication effect did not onset significantly earlier than transition or rounding. Moreover, in absolute time, all three were quite late. The auditory stimulus was not heard until $100 \mathrm{msec}$ after time 0 (trial onset), and it takes $200 \mathrm{msec}$ to plan and launch an eye-movement. Thus, only fixations launched after $300 \mathrm{msec}$ could have reflected any stimulus driven processing. By contrast, we see effects at around 800 msec, quite late into the signal, and near the onset of the vocoid. For comparison, McMurray, Clayards, et al. (2008) found the onset of VOT at 344 and $398 \mathrm{msec}$ using the same procedures at these same thresholds. At the $40 \%$ and $50 \%$ thresholds, the effect of frication occurred slightly, but significantly, later than that of transition and rounding, suggesting the utilization of frication 
may have taken longer to build as well.

\section{Discussion}

Although frication spectrum preceded formant transitions and the vowel by almost 350 msec, the effect of frication did not occur significantly earlier than those of formant transition and rounding. Moreover, at some thresholds the frication effect was significantly later than that of transition and rounding. These null effects are not an artifact of our analysis style: Figure 4 shows clearly coincident effects. Moreover, in Supplement S2 we present analyses which asked whether each effect was individually significant in consecutive $100 \mathrm{msec}$ time windows. This showed a similarly late effect. Thus, Experiment 1 suggests fricatives are processed with a different strategy than other phonemes. Whereas vowels, voicing and manner all show immediate integration, listeners wait to use the frication spectrum to activate lexical entries until other cues (formant transitions) or context (vowel rounding) have arrived.

The timing of the transition and rounding effects may appear to add complexity to this account - the steady state portion of the vowel comes after the transition, but its effects are coincident. We offer two (non-exclusive) explanations. First, if fricative identification waits until all of the relevant information is available (no matter where that information lies), no cue will be usable until all three are available at which point all three factors will be used simultaneously. However, it is also likely that vowel rounding can be identified during the formant transitions: work on "silent center" vowels shows that formant transitions alone are sufficient to cue robust vowel percepts (Jenkins, Strange, \& Miranda, 1994; Strange, Jenkins, \& Johnson, 1983). If this is the case, listeners are simply using both cues as they arrive.

Either way, the most important finding was the extremely delayed use of frication. This 
was an unexpected pattern of results and we were concerned that it may have derived from spurious methodological decisions, not a core use of a memory buffer strategy. Thus, the next four experiments address potential methodological artifacts. Experiment 2 asks if the presence of three sources of information that simultaneously varied in the experiment (increasing task demands) is the cause of these findings. Experiment 3 asks if these findings persist when the fricative is not presented in an utterance-initial position (in a carrier phrase). Experiment 4 validates that there was sufficient information in the frication alone to support fricative identification; and Experiment 5 asks if an artifact of our synthesis methods could explain these results. All four hypotheses are ruled out. Finally, Experiment 6 asks what triggers the late use of frication spectrum.

\section{Experiment 2}

Experiment 2 addresses two methodological factors that could have given rise to the buffer strategy. The first difference between this study and prior work was the presence of a context factor-vowel rounding. With sufficient contextual variability, listeners may wait until they have context to interpret the primary cue. In the prior studies that examined context (Sjerps et al., 2013; Toscano \& McMurray, 2012), the context (a carrier sentence) preceded the primary cue so there was no need to wait for it. Thus, it is unclear whether the presence of following contextual variation may lead listeners to a memory strategy.

Second, most prior studies varied two factors (McMurray, Clayards, et al., 2008; Reinisch \& Sjerps, 2013; Toscano \& McMurray, 2012). In contrast, Experiment 1 varied three highly different factors. Thus, the demands of tracking and integrating an additional factor could have led to the buffered strategy in Experiment 1. Indeed Johnson (1991) showed that when 
listeners are confronted with fricative tokens varying in both talker and vowel rounding across trials, they utilize context differently than when one factor is constant across a block of trials.

Thus, Experiment 2 simultaneously tested these two hypotheses by reducing the number of factors manipulated. We held vowel rounding constant while varying only the frication spectrum and formant transitions. This reduced the number of factors and eliminated contextual variability. If either was responsible for the unexpected cue integration strategy in Experiment 1, participants in Experiment 2 should show immediate integration.

Experiment 2 also modified the filler stimuli to vary both VOT and VL. This was done to verify that our testing procedure and data analysis techniques (even though they were largely based on these prior studies) could detect immediate integration using cues that are known to support it (McMurray, Clayards, et al., 2008; Toscano \& McMurray, 2012).

\section{Methods}

Participants. Twenty-seven adult, monolingual English speakers from Iowa City were recruited in accordance with IRB protocols and received \$15/hour. Participants self-reported normal hearing and normal or corrected-to-normal vision.

Design. Each participant was tested on eight word pairs, four fricative pairs and four stop-consonant pairs. Stop-consonant word pairs were the same for all participants. Participants were tested on either rounded or unrounded fricative word pairs, but not both. Each fricative pair used six frication steps and two formant transitions, while each stop-consonant pair used six VOT steps and two vowel lengths for a total of 96 stimuli. Participants heard each stimulus six times over the course of two separate one hour sessions for a total of 1152 trials. The procedure was the same as in Experiment 1. 
Auditory stimuli. Fricative stimuli from Experiment 1 were used. Voicing continua were created by manipulating natural recordings of bilabial voiced/voiceless minimal pairs: beak/peak, beer/pier, best/bet, and bet/pet. For each pair, a VOT continuum was created by excising 7.5 msec segments of initial voicing from the voiced token, and replacing it with a corresponding amount of aspiration from the voiceless token (McMurray, Aslin, Tanenhaus, Spivey, \& Subik, 2008). This yielded six-step VOT continua from approximately 0 to $45 \mathrm{msec}$ for each word pair. To manipulate vowel length (VL), the vocoid was resynthesized using the Pitch Synchronous Overlap Add (PSOLA) procedure in Praat to extend or contract the VL by $40 \%$.

\section{Results}

Mouse-click responses. We first analyzed the mouse clicks to verify the effect of each factor. As in Experiment 1, fricative categorization (Figure 5A) showed strong effects of frication step, and smaller effects of transition at intermediate steps. Figure 5B indicates that both VOT and VL affected labeling. Stimuli at step 1 were reliably labeled /b/ and those at step 6 as /p/. At intermediate steps there were more clicks on /p/ for short VL.

Mouse click data

were analyzed using a

logistic mixed effects model

similar to the one used in

Experiment 1. However, as

Experiment 2 did not vary

rounding within subjects,

this factor was excluded.
A.

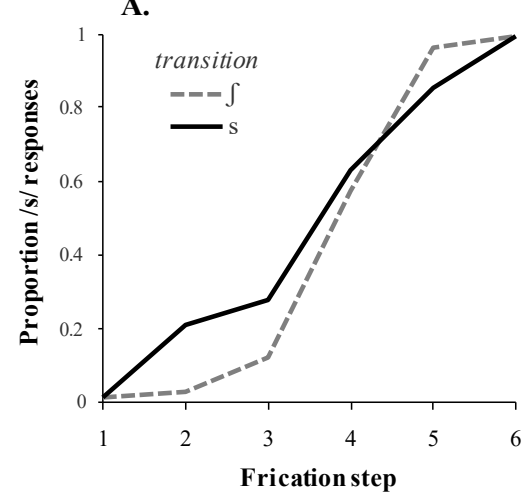

B.

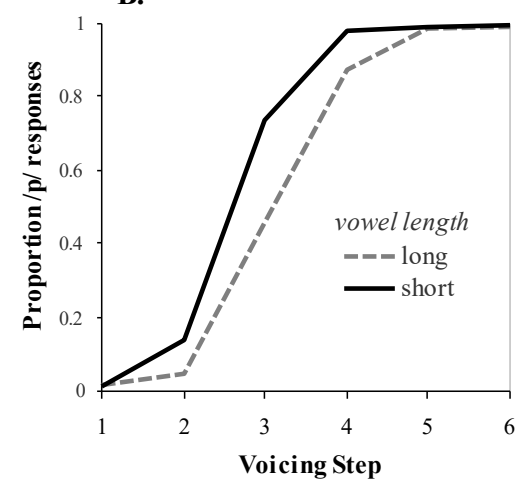

Figure 5. Proportion of mouse clicks for Experiment 2 (A) to the /s/ item as a function of frication step and transition and (B) to the $/ p /$ item as a function of VOT step and vowel length. 
The analysis of fricatives began with a base model including all fixed effects and random intercepts for subject and word pair. Adding random slopes of frication on subject significantly increased fit $\left(\chi_{2}(2)=483.62, p<.001\right)$, as did adding random slopes of frication on word pair $\left(\chi_{2}(2)=271.84, p<.001\right)$, More complex models failed to converge. Therefore, the final model included frication and transition as fixed effects, with random slopes of frication on both subject and word-pair (3).

$$
\text { Response } \sim \text { Step * Transition }+(\text { Step } \mid \text { Subject })+(\text { Step } \mid \text { Word Pair })
$$

Table 5 shows the results. We found main effects of frication $(\mathrm{p}<.001)$ and transition $(\mathrm{p}<.001)$. These were moderated by a frication $\times$ transition interaction $(\mathrm{p}<.001)$, which indicated that identification slopes were shallower for /s/-transitions. Thus, this analysis shows a similar pattern of data to Experiment 1.

Stop-consonant data were analyzed with a similar model. We began with a base model that included fixed effects of VOT and VL and random intercepts for subject and word pair. Adding a random slopes of VOT on subject $\left(\chi_{2}(2)=221.29, \mathrm{p}<.001\right)$ and random slopes of VOT on word pair $\left(\chi_{2}(2)=48.88, \mathrm{p}<.001\right)$ significantly increased fit. More complex models failed to converge. Thus, the final model Table 5. Results of a mixed model examining mouse-click results for fricative and stop-consonant stimuli for Experiment 2. $p>.1$ included VOT and VL as fixed effects, and random slopes of VOT on subject and word-pair (4).

\begin{tabular}{|c|c|c|c|c|c|}
\hline & Factor & B & SE & $\mathbf{z}$ & $\mathbf{p}$ \\
\hline \multirow{3}{*}{ 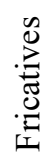 } & Frication & 2.95 & 0.47 & 6.23 & $<.001$ \\
\hline & Transition & 0.52 & 0.06 & 8.07 & $<.001$ \\
\hline & Frication $\times$ transition & -1.1 & 0.07 & -15.54 & $<.001$ \\
\hline \multirow{3}{*}{ 号 } & VOT & 3.24 & 0.22 & 14.89 & $<.001$ \\
\hline & $\mathrm{VL}$ & -1.74 & 0.10 & 17.82 & $<.001$ \\
\hline & $\mathrm{VOT} \times \mathrm{VL}$ & -0.48 & 0.09 & -5.24 & $<.001$ \\
\hline
\end{tabular}




$$
\text { Response } \sim \text { VOT * VL }+(\text { VOT } \mid \text { Subject })+(\text { VOT } \mid \text { Word Pair })
$$

Table 5 shows a significant main effect of VOT $(\mathrm{p}<.001)$ and a significant main effect of VL $(p<.001)$. In addition, there was a significant interaction as curves were shallower for stimuli with long VL $(\mathrm{p}<.001)$. Thus, this analysis showed the expected effects of both VOT and VL.

Evidence of effects in eye- movement data. We determined that there was evidence of effects of each stimulus cue using a similar analysis as that of in Experiment 1 (Supplement S3).

\section{Timing of effects in eye-}

movement data. We analyzed the timing of the effects with the same procedure as Experiment 1 (Figure 6A; Table 6). The effect of frication did not onset earlier than the effect of transition using the $20 \%, 30 \%, 40 \%$ or $50 \%$ thresholds. In contrast, for voicing continua (Figure 6B, Table 6), the effect of VOT onset significantly earlier than VL at the $20 \%$ and $40 \%$ thresholds, and it was marginal at the $30 \%$ threshold. By the $50 \%$ threshold, there was no significant difference between the onset of VOT and VL.

\section{Discussion}

Experiment 2 replicates Experiment 1: participants used frication spectrum simultaneously with the transition. Thus, the buffer strategy does not derive from either the number of manipulated cues nor the presence of context variability. Further, Galle (2014), 
Table 6. Timing of fricative and stop consonant effects in eye-movement data for Experiment 2. $p>.1$ not shown.

\begin{tabular}{ccccccccr} 
& \multicolumn{3}{c}{ Fricatives } & \multicolumn{4}{c}{ Stop Consonant Voicing } \\
& Effect onset (msec) & Frication vs. Trans & Effect Onset (msec) & VOT vs. VL \\
Threshold & Fricative & Trans & $t(26)$ & $p$ & $V O T$ & $V L$ & $t(26)$ & $p$ \\
$20 \%$ & 766 & 814 & 0.14 & & 536 & 620 & 2.14 & .04 \\
$30 \%$ & 846 & 869 & 0.06 & 582 & 673 & 1.82 & .08 \\
$40 \%$ & 907 & 897 & 0.03 & 635 & 716 & 2.14 & .04 \\
$50 \%$ & 972 & 930 & 0.11 & 692 & 749 & 1.06 &
\end{tabular}

replicated Experiment 2 holding transition constant (only manipulating frication and rounding), and showed the same results. This provides further support that the number of manipulated factors was not responsible for the buffer effect. Finally, our replication of the asynchronous use of VOT and VL demonstrated that this pattern for fricatives is not an idiosyncratic property of our sample of subjects or our preparation - we see immediate integration where it is predicted.

\section{Experiment 3: Fricative processing within sentences}

A second explanation for the buffering effects is that sibilant fricatives in isolation do not sound like other speech sounds - they are aperiodic and substantially higher in frequency than other speech sounds. Consequently, the auditory system may not initiate lexical access until it has clearer evidence that the incoming sound is speech. Such a finding could explain the partial evidence for immediate processing in the s/f contrast seen by Mitterer and Reinisch (2013) in word-final fricatives (though as noted other issues that prevent a clear conclusion of immediate processing). Here, the presence of the immediately preceding vowel kicks off lexical access so that lexical access can immediately use the frication. Testing this integration strategy with wordfinal fricatives is not easy, as there are no cues after the fricative with which to compare the onset of the frication effect. Instead, we tested this here by using semantically neutral sentence 
contexts to create conditions under which language processing was ongoing.

\section{Methods}

Participants. 26 monolingual English speaking adults were recruited in accordance with university human subject protocols and received $\$ 15$ per hour. Participants self-reported normal hearing and normal or corrected-to-normal vision.

Design. Four semantically neutral carrier phrases (Table 7) were appended to onset of the same 96 fricative and stop-consonant tokens used in Experiment 2. Pilot work suggested it was not possible to create coarticulatorially neutral sentences. Thus, sentences were recorded in both / $/$ or $/ \mathrm{s} /$ contexts. This resulted in 384 fricative stimuli $(4$ sentences $\times 2$ sentences transitions $\times 4$ continua $\times 2$ word transitions $\times 6$ steps). As in Experiment 2, vowel rounding was constant within a subject. Filler stimuli consisted of four $\mathrm{b} / \mathrm{p}$ continua (from Experiment 2), spliced on to the same four sentences. We did not vary VL, so stop stimuli were not analyzed. This resulted in 192 filler stimuli ( 4 sentences $\times 2$ sentence transitions $\times 4$ continua $\times 6$ steps $)$. Each was repeated twice for 384 trials. This led to a total of 768 trials over two separate one hour sessions spaced between one and ten days apart. The procedure was identical to Experiment 1.

Stimuli. We recorded 5-10 exemplars of each carrier phrases with both /S/- and /s/-initial words in the final position. Carrier phrases were spoken by the same talker as the original words We selected the best exemplar of each sentence and cut the final word. These sentences were then spliced onto the same 96 words used in Experiment 1. In order to create natural-sounding Table 7. Sentences used in Experiment 3 and their durations.

\begin{tabular}{llcc} 
& Sentence & \multicolumn{2}{c}{ Length (msec) } \\
/s/ & /S/ \\
\hline 1 & On this screen, click on the ... & 1800 & 1741 \\
2 & On this display, choose the ... & 1722 & 1885 \\
3 & On this screen, select the ... & 1432 & 1489 \\
4 & On this screen, please choose the ... & 1744 & 1772
\end{tabular}


sentences, we used 20 msec of overlap when

splicing targets onto for Sentence 2 and $40 \mathrm{msec}$ of

overlap for Sentences 1, 3, and 4.

\section{Results}

Mouse-click responses. We first analyzed the mouse clicks. As in Experiments 1 and 2, fricative categorization (Figure 7) showed strong effects of frication step, with intermediate frication

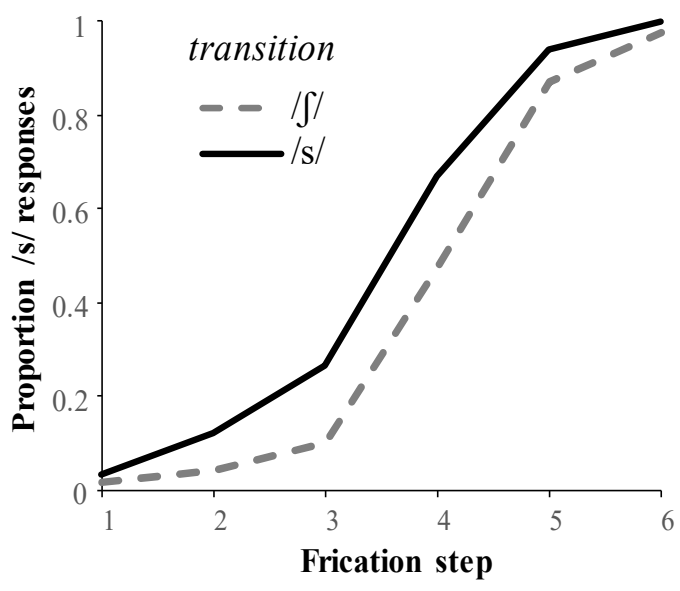

Figure 7. Proportion of mouse clicks for Experiment 3 to the $/$ s/ item as a function of frication step and transition. steps showing smaller effects of transition.

Mouse click data were analyzed using a logistic mixed effects model similar to Experiment 2 with the addition of sentential transition as a fixed effect. Adding random slopes of frication on subject $\left(\chi_{2}(2)=392.04, \mathrm{p}<.001\right)$ significantly increased fit, but more complex models failed to converge. Therefore, the final model included frication step, transition and sentential transition as fixed effects, as well as random slopes of frication on subject (5).

$$
\begin{gathered}
\text { Response } \sim \text { Step } * \text { Transition * Sentence Transition }+(\text { Step | Subject }) \\
+(1 \mid \text { Word Pair })
\end{gathered}
$$

Table 8 shows the results. There was a significant main effect of frication $(\mathrm{p}<.001)$ as well as a significant main effect of transition in the target word
Table 8. Results of a mixed model examining mouse-click results for fricative stimuli for Experiment 3. $p>$.1 not shown.

\begin{tabular}{lrrrr} 
Factor & $\mathbf{B}$ & $\mathbf{S E}$ & $\mathbf{z}$ & $\mathbf{P}$ \\
\hline Frication & 2.54 & 0.19 & 12.44 & $<.001$ \\
Transition & 1.20 & 0.08 & 14.74 & $<.001$ \\
Sentential transition & 0.05 & 0.08 & 0.60 & \\
Frication $\times$ transition & -0.19 & 0.08 & -2.35 & 0.01 \\
Frication x sentential transition & 0.09 & 0.07 & 1.18 & \\
Transition x sentential transition & 0.10 & 0.16 & 0.61 & \\
Frication x trans x sentential trans & 0.13 & 0.15 & 0.87 &
\end{tabular}


$(\mathrm{p}<.001)$. These were moderated by a significant frication $\times$ transition interaction $(\mathrm{p}<.001)$, which indicated that identification slopes were shallower for $/ \mathrm{s} /$-transitions. Sentential transition did not exert an effect. Thus, this analysis shows a similar pattern of data to Experiment 1.

Evidence of effects in eye-movement data. We determined whether there was evidence of effects of each stimulus cue using a similar technique to those used in Experiments 1 and 2 (Supplement S3). We found significant effects of frication and transition, but no effect of the sentence transition. Thus, this latter factor was not analyzed for timing.

Timing of effects in eye-movement data. We next analyzed the timing of the effects using the same procedure as Experiments 1 and 2 (Figure 8, Table 9). The effect of frication did not onset significantly earlier than the effect of transition using the $20 \%, 30 \%$, $40 \%$, or $50 \%$ thresholds (Table 9 ).

\section{Discussion}

This experiment demonstrates evidence for a buffer strategy even in the context of running speech. Carrier sentences were temporally contiguous with the fricative and were intended to alert the speech perception system that the frication was "part of the stream" and that lexical access should be begun. The continued evidence for a buffer

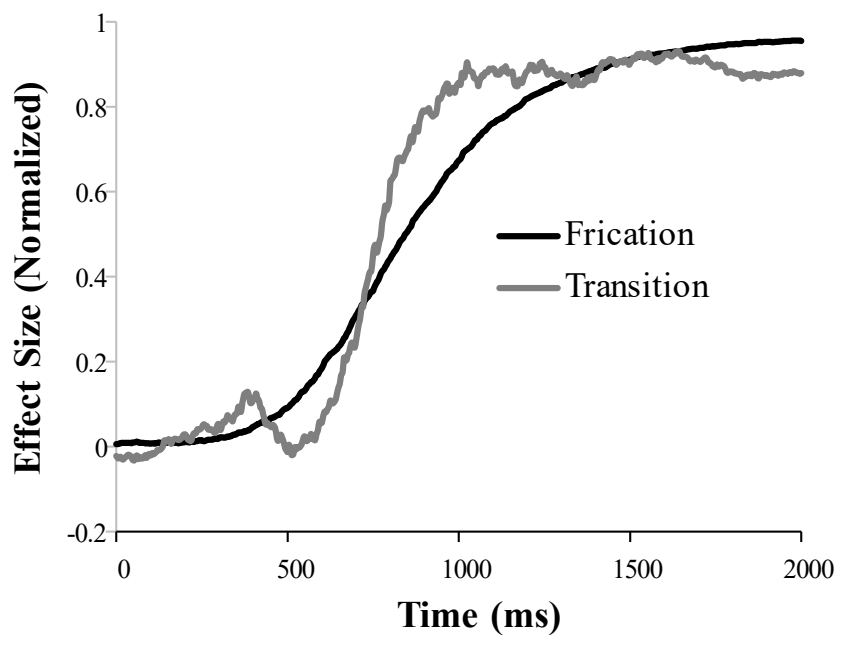

Figure 8. Normalized and jackknifed effect sizes as a function of time for frication and transition in Experiment 3.

Table 9. Timing of fricative effects in eye-movement data for Experiment 3. $p>.1$ not shown.

\begin{tabular}{crrrr}
\multicolumn{5}{c}{ Effect onset (msec) } \\
Threshold & Fricative & Trans & t(26) & p \\
\hline $20 \%$ & 609 & 643 & 0.07 & \\
$30 \%$ & 694 & 714 & 0.31 & \\
$40 \%$ & 769 & 739 & 0.34 & \\
$50 \%$ & 844 & 775 & 0.75 &
\end{tabular}


strategy suggests that the partial evidence for an immediate effect of frication seen in Mitterer and Reinisch (2013) may not have been due to their non-initial position fricatives. Fricatives here were in medial position, and we still find simultaneous effects. We cannot rule out the possibility that lexical access itself needs to be underway for fricatives to show immediate integration (since the sentences were neutral they would not have initiated lexical access for these target words). However, as described this would be difficult to test since there is not another cue following the frication.

\section{Experiment 4: Gated identification}

A third hypothesis is that the artificially generated fricative portions of our auditory stimuli did not contain sufficient information for identification on the basis of the frication alone. Under this view, listeners may have adopted a buffered approach to deal with the lack of bottomup information in the frication. This seemed unlikely given that frication spectrum showed strong effects on mouse click responding. However, to rule this out, Experiment 4 determined listeners' ability to identify gated portions of the stimuli from Experiment 1 in a two alternative forced choice task (Smits et al., 2003; Warner et al., 2014). If there truly was insufficient information in the frication, listeners should be at chance on the gates including only the frication.

\section{Methods}

Participants. 28 adult, monolingual English speakers from the Iowa City area were recruited in accordance with IRB protocols and received class credit for their participation. Three were excluded for non-responses on more than 5\% of trials, leaving 25. Participants self-reported normal hearing and normal or corrected-to-normal vision. 
Stimuli and Design. Auditory stimuli were created by cutting short portions (gates) from the experimental stimuli used in Experiment 1. Four gates were used: 50 percent of the initial fricative (Gate 1), 100 percent of the initial fricative (Gate 2), the entire initial fricative plus four pitch periods of the vowel (Gate 3, $\mathrm{M}=29.2 \mathrm{msec}, \mathrm{SD}=1.1 \mathrm{msec}$ ), and the entire auditory stimuli (Gate 4). The first two gates captured available fricative information, the third captured transition information as well as some information about the lip rounding of the vowel, and the fourth included the entire vowel. All 96 experimental stimuli from Experiment 1 were gated to create 384 auditory stimuli. Each was used on one test trial, in random order (all gates interleaved). Visual stimuli were the same as those used in Experiment 1.

Procedure. On each trial participants heard an auditory stimulus over headphones and saw two pictures corresponding to endpoints of the continuum. The positions of the pictures were random. Listeners pressed a key to indicate which picture matched the auditory stimuli.

\section{Results}

Participants accurately categorized the stimuli, with a category boundary between Step 3 and 4. Figure 9 suggests listeners robustly categorized the fricatives using first half of the frication alone (Gate 1). Transition and rounding did not affect identification until the last gate, at which substantial vocalic information is available.

We assessed this statistically with a logistic mixed model. The dependent variable was binary $(/ \mathrm{s} /$ responses $=1)$. Fixed effects included frication step $(1-6$, centered), rounding (rounded $=+.5$, unrounded $=-.5)$, and transition $(/ \mathrm{s} /=+.5, / \mathrm{J} /=-.5)$. Gate was dummy coded with gate 1 as the reference category. The fixed effects also included two-way interactions of each stimulus factor 

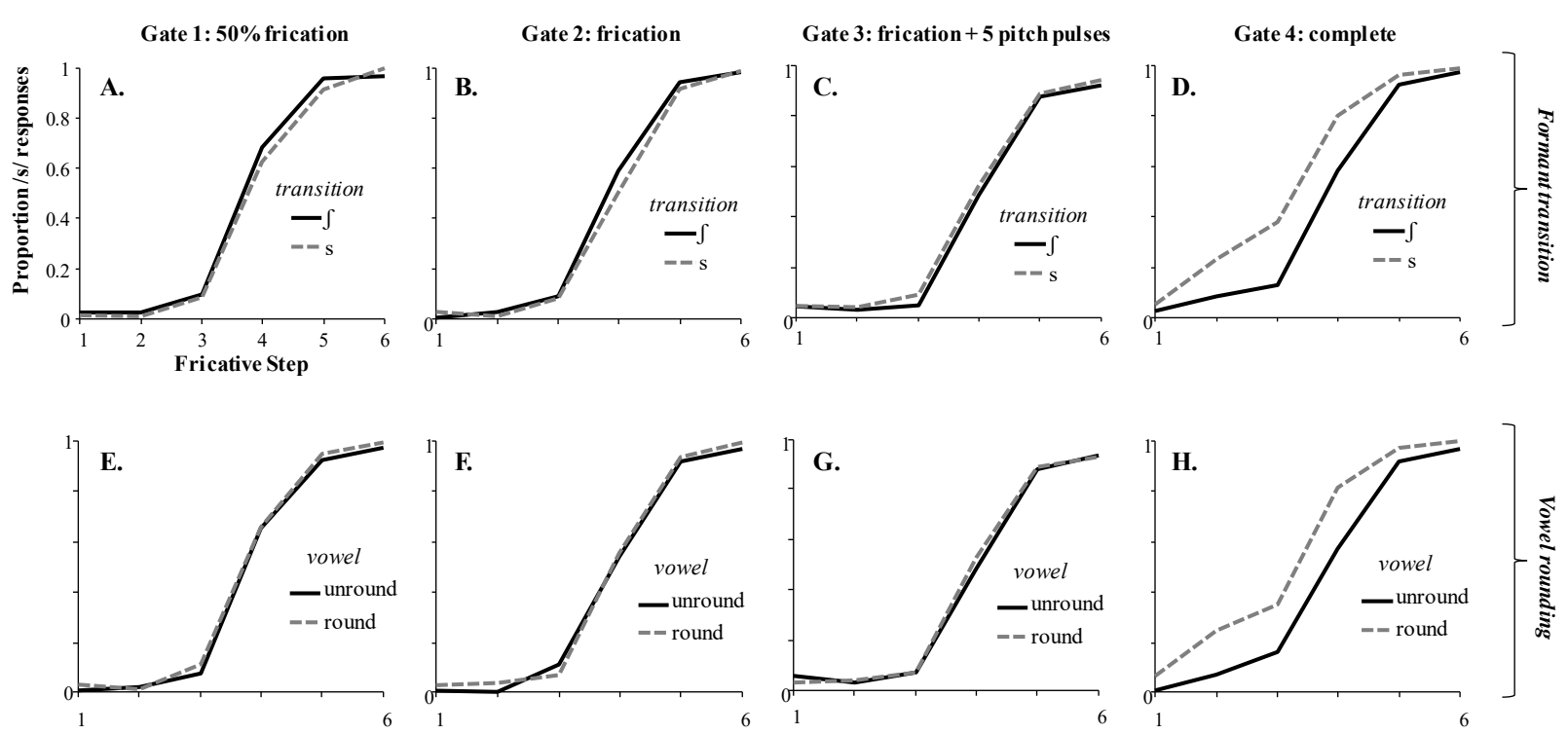

Figure 9. Listeners' labeling of gated stimuli as a function of both step and gate in Experiment 4. Numbers refer to the gate, letters to the condition: A) rounding and B) transition.

(frication, transition and rounding) with gate, but no higher order interactions. Because gate was dummy coded, each interaction term tests the hypothesis that the relevant cue is used differently at later gates than Gate 1, and the main effects should be interpreted as the effect of the given factor at Gate 1. In identifying random effects, the addition of random slopes of frication, transition, and rounding on subject significantly increased fit over a model with subject intercepts alone $\left(\chi_{2}(8)=163.8, \mathrm{p}<.0001\right)$. However, these models only converged when assuming the covariance among random slopes to be zero. Models with additional slopes (gate variables and interactions) did not converge. Therefore, the final model included only random slopes of frication on subject.

$$
\begin{array}{r}
\text { Response }=(\text { Gate } 2+\text { Gate } 3+\text { Gate } 4) *(\text { Step }+ \text { Transition }+ \text { Rounding })+ \\
(1 \mid \text { subject })+(0+\text { Step } \mid \text { Subject })+(1 \mid \text { word pair })
\end{array}
$$


Results are in Table 10. The main effect of frication step was highly significant $(\mathrm{p}<.0001)$. Given the dummy coded gates, this suggests that even at Gate $1(50 \%$ of the fricative), listeners were highly sensitive to frication step. Transition and rounding were not significant as main effects. This was expected since neither factor was present in the stimulus Gate 1
Table 10: Results of mixed model for Experiment 4. $p>$.1 not shown.

\begin{tabular}{lrrrrr} 
& & $\mathbf{B}$ & $\mathbf{S E}$ & $\mathbf{z}$ & $\mathbf{p}$ \\
\hline Gate 2 & & -0.259 & 0.12 & -2.21 & .027 \\
Gate 3 & -0.248 & 0.11 & -2.26 & .024 \\
Gate 4 & 0.824 & 0.11 & 7.73 & $<.0001$ \\
Frication & & 2.477 & 0.15 & 16.05 & $<.0001$ \\
Transition & & -0.277 & 0.16 & -1.73 & .084 \\
Rounding & & 0.235 & 0.17 & 1.36 & \\
Frication & $\times$ Gate 2 & -0.077 & 0.13 & -0.62 & \\
& $\times$ Gate 3 & -0.594 & 0.11 & -5.41 & $<.0001$ \\
& $\times$ Gate 4 & -0.519 & 0.11 & -4.65 & $<.0001$ \\
Transition & $\times$ Gate 2 & 0.065 & 0.22 & 0.30 & \\
& $\times$ Gate 3 & 0.456 & 0.20 & 2.24 & .025 \\
& $\times$ Gate 4 & 1.505 & 0.21 & 7.16 & $<.0001$ \\
Rounding & $\times$ Gate 2 & -0.121 & 0.22 & -0.56 & \\
& $\times$ Gate 3 & -0.181 & 0.20 & -0.89 & \\
& $\times$ Gate 4 & 1.150 & 0.21 & 5.45 & $<.0001$
\end{tabular}

(or 2). Transition became significant at Gate 3 (transition $\times$ Gate 3 interaction, $p=.023$ ) and remained so at Gate $4(\mathrm{p}<.0001)$, suggesting listeners used the transition as soon as it was available. Rounding was only significant at Gate $4(\mathrm{p}<.0001)$, suggesting listeners needed more than 4 pitch pulses (what was available at Gate 3) to identify the vowel and use it for compensation. The effect of frication was tempered at Gates 3 and $4(3: p<.0001 ; p<.0001)$ as the significant negative interactions indicated reduced effects. This is likely due to the additional cues tempering the importance of frication alone.

\section{Discussion}

Experiment 4 verifies that sufficient acoustic information is available within the frication for participants to accurately identify the fricatives at very high levels of accuracy. Participants can categorize the stimuli when forced to do so. However, in the somewhat more realistic 
circumstances of the VWP experiments - when the stimulus is unbroken, there is no time pressure, and listeners are identifying the word's meaning, not its spelling-listeners appear wait to use that information for lexical access.

\section{Experiment 5: Naturally produced fricatives}

Experiment 4 demonstrated there is sufficient information in the frication alone to determine place of articulation. However, it is possible the rather artificial nature of the stimuli is what drove listeners toward the buffer strategy. Indeed prior work on cue weighting suggests differences in natural and synthetic speech (Joanne L. Miller \& Wayland, 1993; Shinn, Blumstein, \& Jongman, 1985; Utman, 1998). This is unlikely to determine the effects here, as prior work on temporal integration in stop voicing suggests that the naturalness of the stimuli does not affect whether or not listeners adopt an immediate integration or memory-based strategy (Toscano \& McMurray, 2012). However, it is unclear whether this extends to fricatives.

Thus, Experiment 5 investigated the time course of cue integration for completely natural stimuli. If listeners still wait until the vocoid to activate lexical entries, this would suggest a memory-buffer may be the norm for fricatives. However, if listeners exhibit immediate integration, then the evidence for a memory based strategy mode observed in Experiments 1-3 may derive from stimulus factors.

Experiment 5 did not manipulate the formant transitions or frication spectrum. Thus, the relevant contrast in this experiment is not between the onset of two cues within the fricative. Rather, the primary question was whether listeners distinguish the two fricatives later than they can distinguish the two stop consonants. If listeners continue to show a memory buffer strategy for fricatives, the influence of fricative identity $(/ \mathrm{s} / \mathrm{vs} / \mathrm{J} /$ ) on fixations should be later than that of 
stop-voicing (/g/ vs. /k/).

\section{Methods}

Participants. 26 monolingual adults were recruited in accordance with IRB protocols and received \$15 per hour. Participants self-reported normal hearing and normal or corrected-tonormal vision.

Design and Procedure. Stimuli were derived from the same eight fricative and voicing minimal pairs used in prior experiments. Each of the 16 fricative stimuli and the 16 stopconsonant stimuli were presented ten times, for 320 trials (approximately 30 minutes).

Stimuli. Fricative and stop stimuli consisted of naturally recorded tokens originally recorded as the basis of the synthesized tokens or fillers used in Experiment 1.

Stimuli were unmanipulated with one exception: we equated the length of the fricatives to enable a more precise timelocking of the analysis to the stimulus Phonetic work (McMurray \& Jongman, 2011) suggests that length plays a minimal role in the $s / \int$ distinction. Thus, this was expected to have no influence on the place of articulation decision. To equate length, we measured the length of frication for each exemplar of each word, and selected the subset of the stimuli that minimized variability in fricative length. Once this set was chosen, small $(<5 \mathrm{msec})$ portions of frication were either removed or duplicated at the center of the frication to achieve a frication length of $245 \mathrm{msec}$.

\section{Results}

Mouse-click responses. Participants clicked the correct item on 99.3\% of trials for /s/ targets, $98.7 \%$ for $/ \mathrm{J} /, 99.5 \%$ for $/ \mathrm{k} /$, and $99.4 \%$ for $/ \mathrm{g} /$. Given this high accuracy inferential statistics were not conducted. 


\section{Timing of effects in eye-movement}

data. As in earlier experiments, we calculated

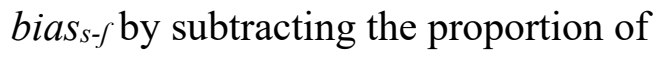

fixations to the $/ \mathrm{J} /$ item from the $/ \mathrm{s} /$ item on

fricative trials (at each 4 msec timeslice).

Similarly, we computed bias $-k$ by subtracting

the proportion of fixations to the $/ \mathrm{k} /$ item from

the /g/ item for stop trials. We converted this

to an effect size by subtracting bias $_{s-\varsigma}$ when

the stimulus was an $/ \mathrm{J} /$ from the bias $_{s-f}$ when

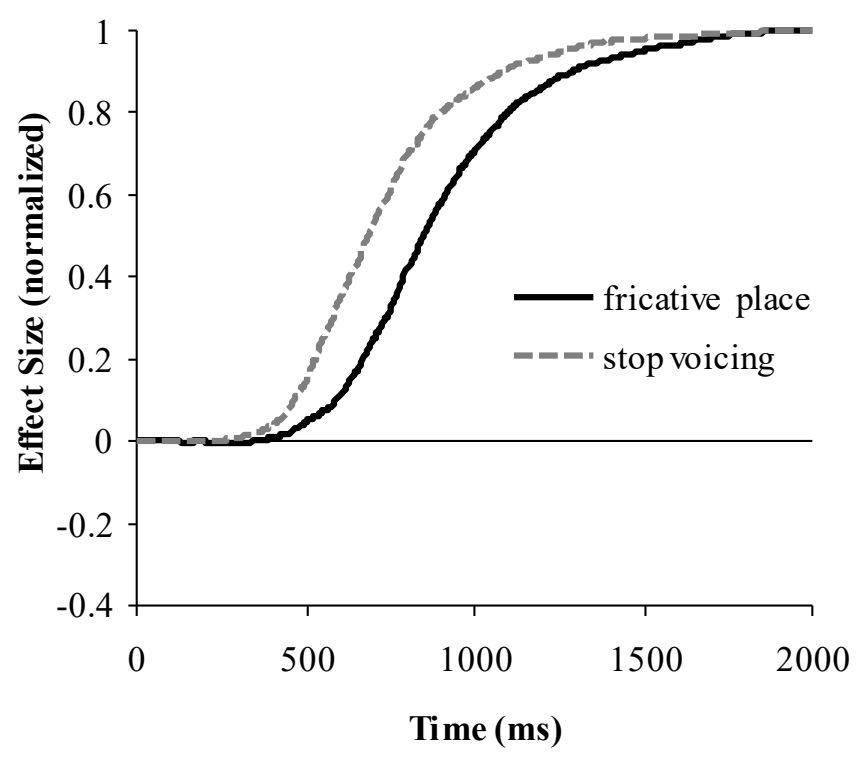

Figure 10. Normalized effect size of fricative place discrimination and stop voicing discrimination as a function of time for Experiment 5. the stimulus was an /s/ (and

similarly for the $\mathrm{g} / \mathrm{k}$ continuum).

These measures represent the

effect of all cues present in the
Table 11. Timing of fricative and voicing effects for Experiment 5.

\section{Effect onset (msec)}

\begin{tabular}{cccrr} 
Threshold & Fricative & Voicing & $\mathbf{t ( 2 5 )}$ & $\mathbf{p}$ \\
\hline $20 \%$ & 673 & 529 & 10.35 & $<.001$ \\
$30 \%$ & 740 & 581 & 12.02 & $<.001$ \\
$40 \%$ & 792 & 633 & 10.77 & $<.001$ \\
$50 \%$ & 852 & 687 & 14.62 & $<.001$
\end{tabular}

stimuli to discriminate the fricatives or stops.

Figure 10 shows that listeners categorize stop-consonants earlier than fricatives. Listeners distinguished stop-consonant stimuli significantly earlier than fricatives at all thresholds (Table 11), with latency differences exceeding $100 \mathrm{msec}$ and even $150 \mathrm{msec}$ at some thresholds.

\section{[At early points] do listeners even know that the stimulus is a fricative?}

We next asked whether the delay observed above is a delay in processing specific acoustic cues for features like place of articulation, or is it a global delay in lexical access? If it is the former, one might predict that even though listeners may wait (until the vocoid) to decide which fricative it is, they may access lexical entries for both fricative options, since it the input is 

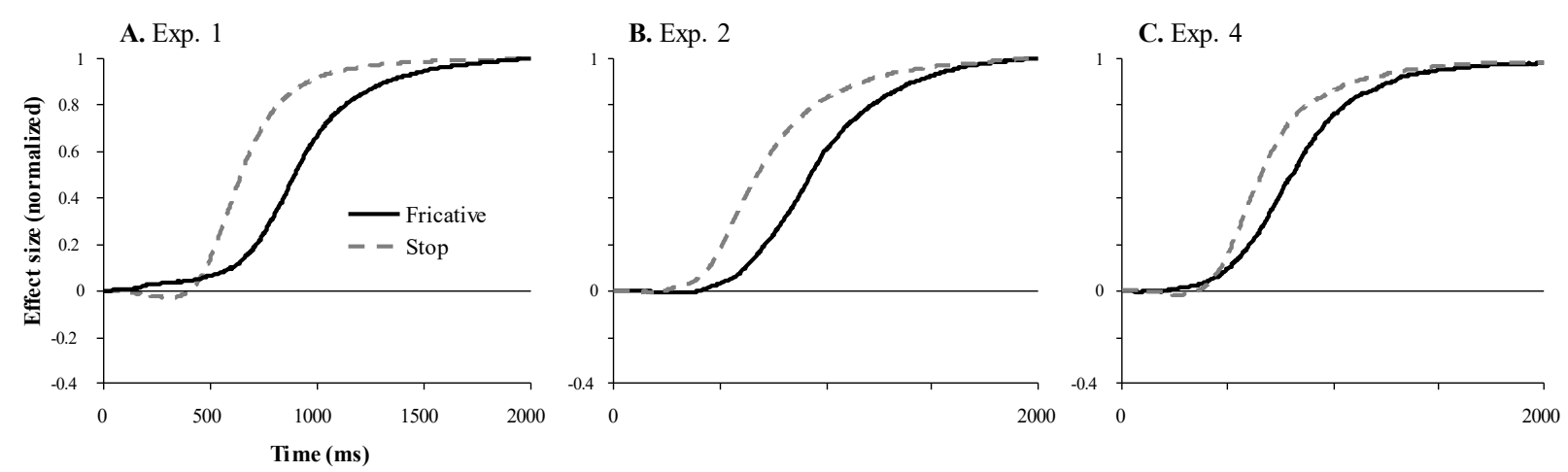

Figure 11. Normalized effect size over time for discriminating fricatives from stop when the stimulus was a fricative or a stop. A) Experiment 1;B) Experiment 2; and C) Experiment 5.

clearly not a stop consonant. In contrast, if listeners simply delay lexical access for fricative inputs, we may find that during the early portion of the signal, they don't commit differentially to fricatives or stops.

To address this, we reanalyzed the data from Experiments 1,2 and 5. We calculated a fricative bias by subtracting the looks to any stop-consonant item from looks to any fricative item, for trials with fricative auditory stimuli. This measure allowed us to ask, for trials in which the stimulus was a fricative, how rapidly did listeners converge on either fricative (ruling out stops). Similarly, we calculated a stop-consonant bias by subtracting the looks to any fricative item from looks to any stop item, for trials with stop stimuli. These two were then compared using the onset detection analyses used in the prior studies.

Figure 11 and Table 12 show the results. Listeners delay lexical access substantially for fricatives, determining that a stimulus was a fricative later than they could tell it was a stop. This was seen across all experiments at the $20 \%, 30 \%, 40 \%$ and $50 \%$ thresholds (Table 12). For stop stimuli, they narrowed lexical access to stops approximately $200 \mathrm{msec}$ before they could do the converse for fricatives.

\section{Discussion.}



$\begin{array}{ll}\text { Experiment } 5 & \text { Table 12. Onset of fricative or stop effects for Experiments 1, 2, } 5 . \\ p>\text {.1 not shown. }\end{array}$

demonstrated that listeners

were able to discriminate the

two stop-consonant stimuli

significantly earlier than

fricatives. Thus, listeners

wait to categorize fricatives

even with natural stimuli.

Furthermore, and somewhat

counterintuitively, during the

\begin{tabular}{cccccc}
\multicolumn{5}{c}{ Effect Onset (msec) } \\
& Threshold & Fricative & Stop & t(26) & p \\
\hline \multirow{2}{*}{$\begin{array}{c}\text { Experiment } \\
1\end{array}$} & $20 \%$ & 716 & 528 & 13.89 & $<.001$ \\
& $30 \%$ & 782 & 566 & 15.49 & $<.001$ \\
& $40 \%$ & 845 & 604 & 18.76 & $<.001$ \\
Experiment & $50 \%$ & 894 & 645 & 18.61 & $<.001$ \\
\hline 2 & $20 \%$ & 717 & 516 & 11.87 & $<.001$ \\
& $30 \%$ & 800 & 565 & 12.86 & $<.001$ \\
& $40 \%$ & 872 & 622 & 16.65 & $<.001$ \\
Experiment & $50 \%$ & 932 & 681 & 22.88 & $<.001$ \\
5 & $20 \%$ & 605 & 524 & 16.57 & $<.001$ \\
& $30 \%$ & 683 & 571 & 15.71 & $<.001$ \\
& $40 \%$ & 743 & 613 & 22.35 & $<.001$ \\
& $50 \%$ & 809 & 663 & 16.85 & $<.001$
\end{tabular}

initial portions of the signal

listeners knew they were hearing a stop-consonant (when the stimulus was a stop) before they knew they were hearing a fricative (when it was a fricative). This was remarkably consistent, with evidence in each study and at every threshold. It is also asymmetric. Given that every trial contained only stops and fricatives, if there was sufficient information to identify that a stop was present (and a fricative was absent), then there should have been sufficient information for the converse. While we are not claiming that lexical access operates by identifying binary features (e.g., stop vs. fricative), apparently listeners do not make a decision about whether the sound they are hearing is a fricative or not until much later than stop-consonants. In effect, when the stimulus is a fricative, they delay lexical access entirely until they have substantial acoustic information.

One loose end is the fact that the timing of the use of cues in the fricative appeared about 100 msec earlier in Experiment 5 than in the other experiments (though it was still prior to the 
use of cues for stop consonants). However, it is important to point out that the fricatives in this experiment were roughly $100 \mathrm{msec}$ shorter $(\mathrm{M}=245 \mathrm{msec})$ than in prior experiments $(\mathrm{M}=350)$. This suggests that the timing of the use of frication may be precisely times to the onset of the vocoid (or the offset of the fricative). Listeners are not merely delayed - they may be waiting for the cues in the vocoid. This prediction was tested in Experiment 6.

\section{Experiment 6: What are listeners waiting for?}

The previous experiments suggest that listeners integrate information for fricative place of articulation using a memory buffer strategy. Listeners are delayed in using information in the frication to decide both the place of articulation and even that the sound was a fricative at all.

But what are they waiting for? One possibility is that listeners are delayed simply because they need more time to process information in the frication. Under this view, they would show immediate integration, except for the fact that it takes several hundred milliseconds to extract the relevant information from the frication. As a result, processing looks buffered. This seems somewhat unlikely as fricatives can be identified with high accuracy from relatively steady-state spectral information (as in Experiment 2 at Gate 1). Alternatively, listeners may wait for the vocoid to begin lexical access (Diehl, Kluender, Foss, Parker, \& Gernsbacher, 1987). This would be consistent with a true memory-integration account and would reveal what type of input causes information to be released from the buffer.

To address this question, Experiment 6 used fricatives that were lengthened or shortened, altering the vocoids' onset. If the onset of the fricative effect is the same across frication lengths, this would suggest listeners require more time to process fricative information but they are not waiting for any specific event. However, if the onset of the fricative effect is delayed with a longer fricatives, this would suggest that they are waiting for additional cues to release 
information from the memory buffer and begin activating lexical candidates.

\section{Methods}

Participants. 45 monolingual adults from the Iowa City area were recruited in accordance with IRB protocols and received $\$ 15 /$ hour. Because we manipulated an additional factor (length), we tested more participants than previous studies. Participants self-reported normal hearing and normal or corrected-to-normal vision.

Design and Procedure. Each participant was tested on eight word pairs: four fricative pairs and four stop pairs. The stop pairs were the same for all participants. Similar to Experiments 2 and 3, participants were tested on either rounded or unrounded fricative word pairs, but never both. To keep the study duration similar to Experiments 1-3, while including an additional variable (fricative length), fricative continua were reduced to five steps instead of six. There were two possible formant-transitions, and two lengths ( 4 continua $\times 5$ steps $\times 2$ transitions $\times 2$ lengths) for 80 stimuli. These were heard 9 times for 720 trials. Stop-consonant pairs had five possible VOTs and two VLs ( 4 continua $\times 5$ steps $\times 2$ lengths $)$ for 40 stimuli. These were heard 18 times for 720 filler trials. The resulting 1440 trials tested in two one hour sessions, spaced between one and ten days apart. 
Stimuli. Auditory

stimuli were based on

Experiment 1. These were

lengthened and shortened

during resynthesis. Once

the filtered noise was

created, the frications were

lengthened by adding or
A.

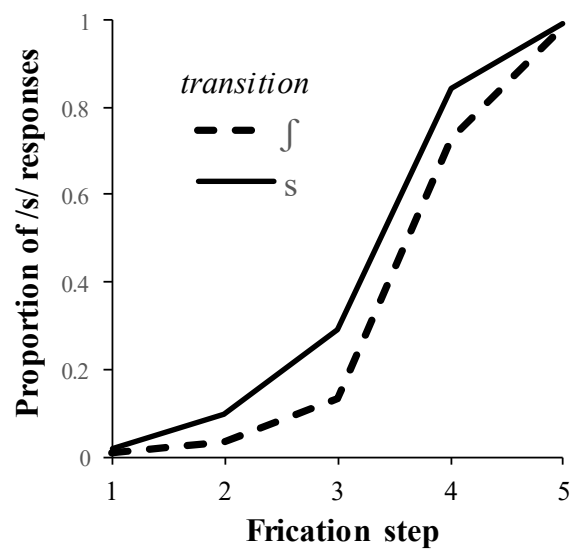

Figure 12. Experiment 6. A) Proportion of mouse clicks to the $/ s /$ item as a function of frication step and transition, and B) frication step and length. C) Mouse clicks to the $/ p /$ item as a function of VOT and VL.

subtracting $50 \mathrm{msec}$ of

steady-state to the center of the envelope before it was imposed on the filtered noise. Stop consonant stimuli were those used in Experiment 2.

\section{Results}

Mouse-click responses. Figure 12A indicates that listeners reliably labeled step 1 of the fricative continuum as $/ \mathrm{s} /$ and step 6 as $/ \mathrm{J} /$. At intermediate steps, there were more $/ \mathrm{s} /$ responses for /s/ formant transitions. Figure 12B suggests that, as expected, length did not affect labeling. For analysis of VOT continua (which showed all the expected effects), see Supplement S4.

Mouse click data for fricative stimuli were analyzed using a logistic mixed effects model similar to Experiments 2 and 3, with the addition of length as a fixed effect (long $=-.5 /$ short $=$ $+.5)$. We started with a base model that included all of the fixed effects and random intercepts for subject and word pair. Adding random slopes of frication on subject significantly increased fit $\left(\chi_{2}(2)=805.05, p<.001\right)$, but more complex models failed to converge. The final model included frication step, transition and length as fixed effects, with random slopes of frication step on 
subject and random intercepts for word pair (7).

$$
\text { Response } \sim \text { Step * Transition * Length }+(\text { step } \mid \text { subject })+(1 \mid \text { word pair })
$$

As Table 13 shows, there were significant main effects of frication $(p<.001)$, with more $/ \mathrm{s} /$ responses at higher steps; and transition $(\mathrm{p}<.001)$ with more $/ \mathrm{s} /$ responses for $/ \mathrm{s} /$ transitions. There was also a small effect of length $(\mathrm{p}=.007)$, with more clicks to /s/ for longer stimuli; as well as significant interactions of frication $\times$ transition and transition $\times$ length.

Evidence of effects in eye-movement data. We

determined whether there was

evidence of effects of each

stimulus cue using a similar

technique to those used in

Experiments 1-3 (Supplement

S3). There were significant
Table 13. Results of a mixed effects model for identification data in Experiment 6. $p>.1$ not shown.

\begin{tabular}{lrrrr} 
Factor & B & SE & \multicolumn{1}{c}{$\mathbf{z}$} & $\mathbf{p}$ \\
\hline Frication & 3.71 & 0.20 & 18.89 & $<.001$ \\
Transition & 1.33 & 0.05 & 24.33 & $<.001$ \\
Length & 0.14 & 0.05 & 2.70 & .007 \\
Frication $\times$ transition & -0.41 & 0.06 & 7.04 & $<.001$ \\
Frication $\times$ length & -0.02 & 0.06 & 0.30 & \\
Transition $\times$ length & -0.31 & 0.06 & 0.30 & .004 \\
Frication $\times$ length $\times$ transition & 0.11 & 0.11 & 0.94 & \\
\hline VOT & 2.40 & 0.21 & 11.21 & $<.001$ \\
VL & 1.17 & 0.06 & 19.17 & $<.001$ \\
VO. & 0.09 & 0.07 & 1.36 &
\end{tabular}

effects of frication step, transition, and rounding. There was also an unexpected small effect of length.

Timing of effects in eye-movement data. Our analysis of the onset of the effects of each cue focused on the use of frication spectrum (as the delay in its use was the primary unexpected finding). The timing of frication/transition and VOT/VL effects were similar to those found in Experiments 1-3 (Supplement S5). In this experiment, our primary concern was whether the use of the frication spectra differed for long and short stimuli. The analysis (Figure 13A, Table 14) confirms this, demonstrating a significantly earlier effect of frication for short stimuli than long 
A. Effect of frication

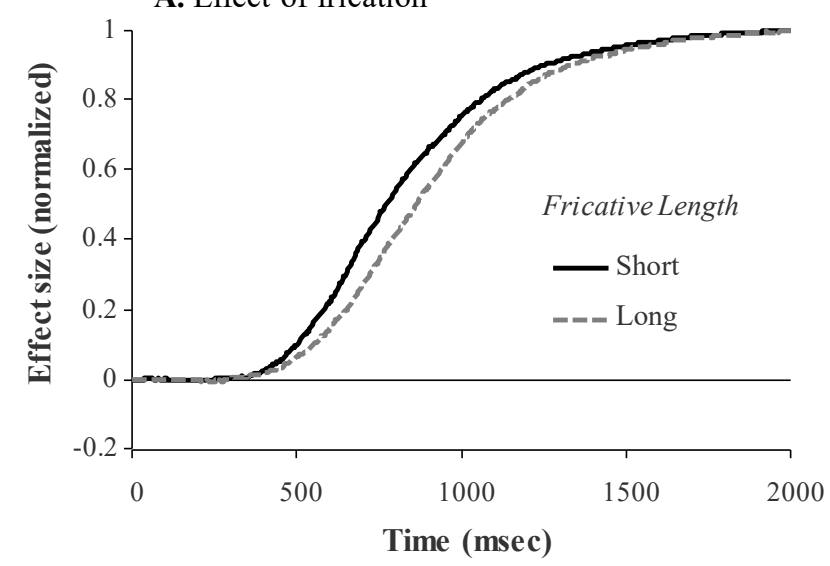

B. Effect of transition

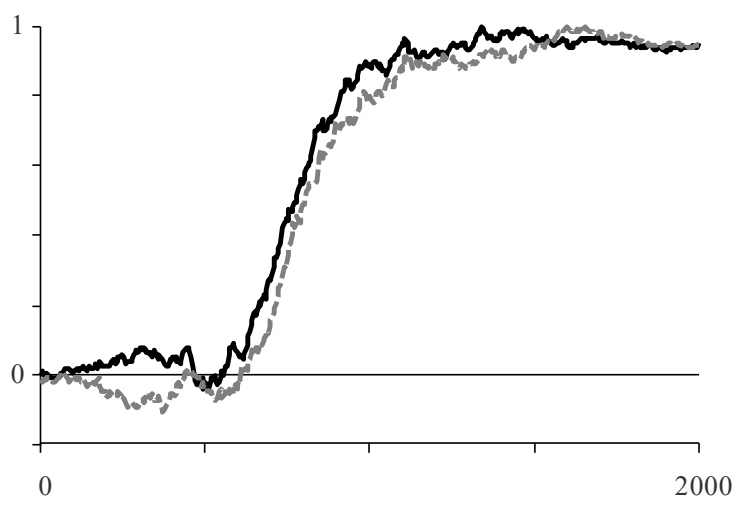

Figure 13. Effect of (A) frication length and (B) transition over time for long and short fricative stimuli in Experiment 6.

stimuli at all thresholds. Secondarily, we examined the onset of the transition effect (Figure 13).

In contrast to the frication effects, there was no significant difference between the transition onset for short and long stimuli at any threshold (Table 12), though effects were all numerically in the right direction. This may simply reflect the relatively small absolute size of the effect (making it more difficult to affect it with the length manipulation). These results indicate that listeners wait until the end of the fricative or until an additional cue is available to start processing the fricative.

We note that across words, the fricatives also varied in length (to preserve naturalness). This offered an additional test of the length effect, by assessing whether there is a relationship between the overall length of individual words and the onset of the frication effect. If the timing

Table 14. Timing of frication and transition effects in eye-movement data for short and long fricative stimuli in Experiment 6. $p>.1$ not shown.

\begin{tabular}{|c|c|c|c|c|c|c|c|}
\hline \multirow[b]{3}{*}{ Threshold } & \multicolumn{4}{|c|}{ Fricative effect } & \multicolumn{3}{|c|}{ Transition effect } \\
\hline & \multicolumn{2}{|c|}{ Effect onset (msec) } & \multicolumn{2}{|c|}{ Short vs. Long } & \multicolumn{2}{|c|}{ Effect Onset (msec) } & \multirow{2}{*}{$\begin{array}{l}\text { Short vs. Long } \\
g \quad t(44)\end{array}$} \\
\hline & Short & Long & $t(44)$ & $\mathbf{p}$ & Short & Long & \\
\hline $20 \%$ & 583 & 654 & 4.84 & $<.001$ & 669 & 717 & 0.88 \\
\hline $30 \%$ & 650 & 724 & 5.86 & $<.001$ & 708 & 743 & 0.80 \\
\hline $40 \%$ & 707 & 792 & 5.77 & $<.001$ & 732 & 767 & 1.00 \\
\hline $50 \%$ & 774 & 865 & 7.39 & $<.001$ & 777 & 805 & 0.59 \\
\hline
\end{tabular}


of the frication effect was tied to the onset of the vocoid, we should also observe that words with shorter fricatives ought to also have earlier effect onsets. Thus, for each word ( 8 word-pairs $\times 2$
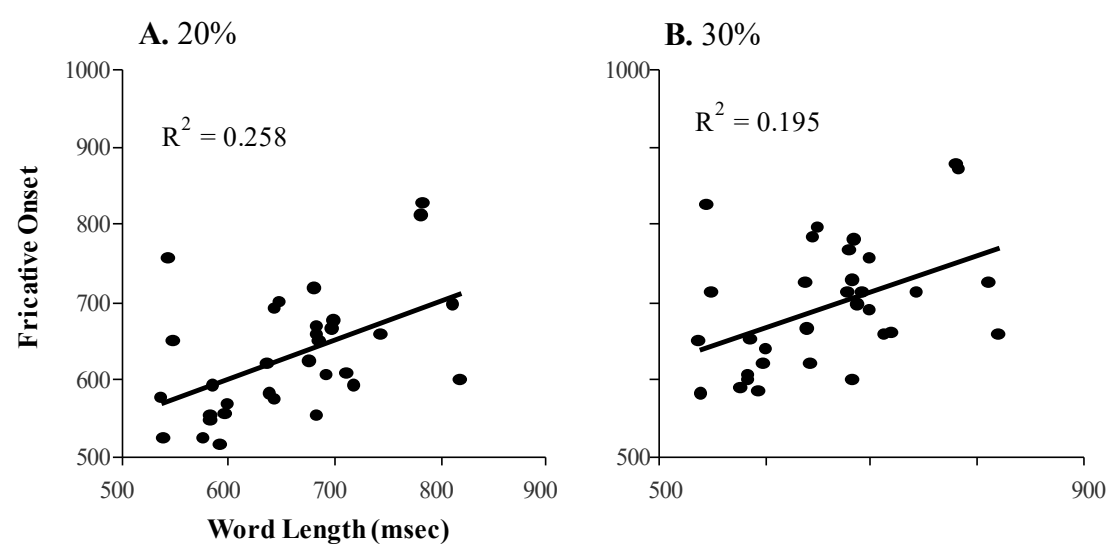

transitions $\times 2$ lengths $=32$ words)

we calculated the correlation between length of frication and the onset of the frication effect for that word (Figure 14). We found a significant correlation at the
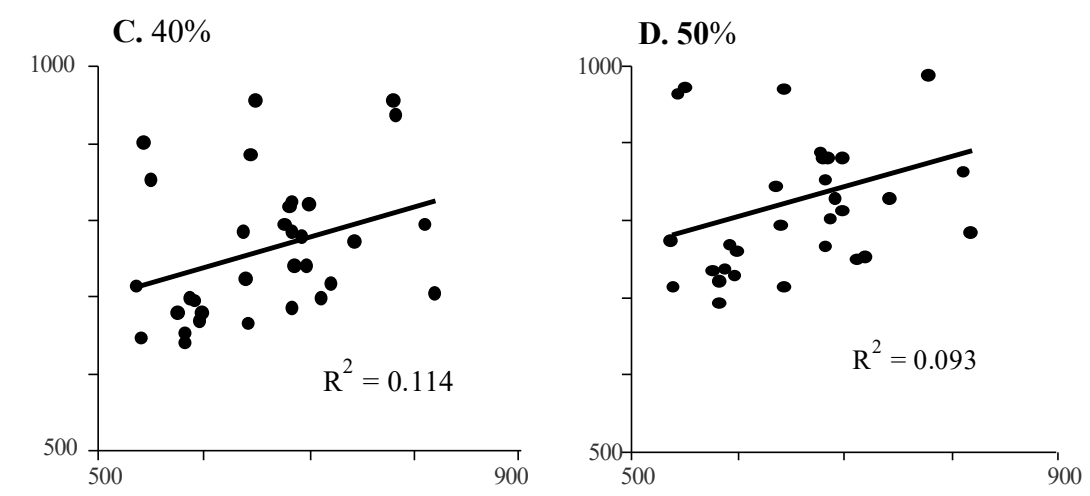

Figure 14. Correlation between length of word and the onset of the frication effect in Experiment 6 at the 20\% threshold (A), 30\% $20 \%(\mathrm{r}(30)=.51, \mathrm{p}=.0029)$ and threshold (B). 40\% threshold (C), and 50\% threshold (D).

$30 \%$ thresholds $(\mathrm{r}(30)=.44, \mathrm{p}=.011)$ and marginally significant correlations at the $40 \%$

$(\mathrm{r}(30)=.34, \mathrm{p}=.059)$ and $50 \%$ thresholds $(\mathrm{r}(30)=.31, \mathrm{p}=.089)$. This provides supporting evidence that listeners do store the fricative in memory until it ends or cues in the vocoid are available.

\section{Discussion.}

Experiment 6 showed a significant difference between the onset of the frication effect for long and short fricatives and a close timelocking to the specific length of the fricative. This suggests that listeners do not merely take longer to process fricatives. Rather, listeners wait to use frication spectrum for lexical access either until the end of the fricative or until other cues, such as formant transition or vowel rounding, are available. 


\section{General Discussion}

This study asked how listeners integrate asynchronous cues to fricatives. We expected listeners to use the same immediate integration strategy for fricative place of articulation as stops and vowels. However, the present experiments show that listeners do not, and employ a memory buffer strategy. In Experiment 1, frication spectrum was used at roughly the same time as transition or vowel rounding, even though it is available earlier. Experiment 2 showed that this was not due to the fact that three sources of information varied in the experiment, nor was it due to the presence of contextual variation (vowel rounding). Experiment 3 showed that delays in use of frication spectrum persisted even when the fricative was not utterance-initial. Experiment 4 showed there was sufficient information in the frication to allow for identification. Even with only $50 \%$ of the frication, listeners could successfully identify the fricative when forced to. Experiment 5 verified that the unexpected results did not derive from the synthetically produced stimuli. Experiment 6 manipulated the length of the frication, showing that listeners wait to begin lexical activation until the onset of the vocoid. Perhaps most surprisingly, across Experiments 1,2 and 5, not only did listeners wait until the vocoid to identify which fricative it was, while they were waiting, there was no evidence that they knew it was a fricative at all.

\section{Limitations}

Before turning to the implication of these findings, three limitations are worth discussing.

First, we point out that these studies may appear to conflict with several prior studies of fricatives. For reasons of brevity we offer a complete discussion in Supplement S6. Briefly, prior gating studies (Smits et al., 2003; Warner et al., 2014) suggest that even at short gates, 
listeners are highly accurate at identifying fricatives. However, as we argued in Experiment 4 listeners may be capable of making a decision when forced to, even if they do not do so in interrupted processing. A more serious discrepancy arises from Kingston et al. (2016), who show some evidence of early use of frication in the easier /s/ vs. /f/ contrast. However, they did not perform the kind of analysis needed to compare the use of frication to other cues, and there are numerous methodological issues that may undercut the claim of immediate integration (see Supplement S6).

Second, these experiments only studied two of the eight fricatives of English (/f, v, $\ominus$, , $\mathrm{s}, \mathrm{z}, \int, 3 /$ ). Our results challenge the assumption that any speech sound can be treated as representative of all speech sounds. Thus, our findings may not generalize to fricative processing in general, but rather may only apply to voiceless fricatives or sibilants or some subset. It will be important to study other fricatives to test whether this buffer strategy is used more generally.

Third, we cannot rule out two common concerns with the VWP as contributors to our effects. In common versions of the VWP (as used here), listeners see the pictures before hearing the word. This minimizes the role of visual search for post-stimulus fixations (since the listener knows where each picture is). However, this pre-scanning could allow listeners to pre-activate lexical items prior to word onset, and influence lexical activation. Similarly, participants, only see a small set of referents on any trial and over the experiment, which might allow them to begin lexical activation earlier than in open-set tasks. Several studies have addressed these concerns, showing effects of non-displayed competitors (Dahan, Magnuson, Tanenhaus, \& Hogan, 2001; McMurray et al., 2009) or effects of visual similarity (ruling out that only the prenames matter: Dahan \& Tanenhaus, 2005), yet such concerns linger.

Our findings are unlikely to derive from such effects for two reasons. First, pre-naming 
and closed set concerns predict earlier activation, rather than the delay we found. Thus, these standard criticisms of the VWP make a stronger case for our findings: even in a task in which lexical activation could be initiated early, listeners still waited for the vocoid. Second, Experiment 2 finds immediate integration for VOT, but buffered integration for fricatives in the same task. This makes it unlikely that our effects derive from the task.

\section{Are sibilant fricatives special?}

Given the fact that immediate integration is shown by stop consonants and approximants (McMurray, Clayards, et al., 2008) and vowels (Reinisch \& Sjerps, 2013) as well as with contextual factors like speaking rate (Toscano \& McMurray, 2015), and perceptual retuning (Mitterer \& Reinisch, 2013), one immediate question is whether sibilant fricatives are unique in this regard? Without research on other speech sounds, any discussion must remain speculative, but there are several points that are relevant.

First, the most salient properties of sibilants might be that 1) they are aperiodic (and thus may not sound entirely speech like in isolation); 2) they are found in much higher frequency bands than other phonemes; and 3) they are clearly segmentable (in time) from the vowel as they are acoustically discontinuous. This latter factor contrasts with approximants $(/ 1 /, / r /)$ that are also long, but blend with the vowel continuously. As we describe next, these properties motivate a potential role for auditory grouping factors. If this is the case, then one might expect that other voiceless fricatives $(/ \mathrm{f} /, / \Theta /)$ or stops with long periods of aspiration (e.g., voiceless stops in Navaho) will show similar properties. It is less clear whether voiced fricatives would show the same properties - they too share aperiodic components, and a higher frequency band with /s/ and $/ \int /$, but the presence of low frequency voicing may serve to bind the fricative with the vowel 
better. It would be fruitful to investigate this more systematically in future work.

\section{Why do listeners wait?}

These caveats aside, these experiments lead to the unexpected but unambiguous conclusion that listeners use a buffer strategy to integrate asynchronous cues for fricatives. It is not clear why. Several possible explanations are worth considering.

Cue Reliability. A critical (though somewhat orthogonal) issue in cue integration is how much to weight individual cues (Nearey, 1997; Oden \& Massaro, 1978; Toscano \& McMurray, 2010). Here a recent idea is that cues get weighted by how reliable they are (Toscano \& McMurray, 2010) (c.f., Ernst \& Banks, 2002; Jacobs, 2002). Could this account for our results?

A first order cue reliability effect is unlikely - the frication spectrum is a far more reliable and useful cue than the formant transitions. This is seen in statistical measurements of the cues for sibilant fricatives (Jongman et al., 2000; McMurray \& Jongman, 2011), and in the perceptual results here. For example in Experiment 1 the effect of transition is only observed at the boundary, and even near the boundary can only shift the percept by $20-30 \%$, whereas the frication spectrum can shift the percept from 0 to $100 \%$, even with unambiguous transitions.

However, a second order effect may be possible by which listeners realize that the frication spectrum occasionally mismatches the transition (e.g., they hear an /s/ frication with an /S/ transition). We cannot rule this out, but it is hard to see how this would lead listeners to down weight the frication (the dominant cue) rather than ignore the transition (a weak secondary cue). And somehow by the end of processing they would need to re-upweight it (to account for the identification results). Moreover, it is not clear that cue weighting should affect when cues are used - in prior studies weak secondary cues like sentential rate (Toscano \& McMurray, 2015) or 
the F2 for a vowel duration contrast (Reinisch \& Sjerps, 2013) have been shown to be used before the primary cues (VOT, duration). Thus, it seems unlikely that a reliability account can explain our results.

Context. A second possibility is that listeners use a memory buffer with fricatives because fricatives are more context dependent and listeners need the vocoid to compensate for talker and coarticulation. This would seem to conflict with findings of immediate integration in vowels (Reinisch \& Sjerps, 2013) where context (particularly talker) may be even more of a factor. However, in vowels the relevant context (F0) is simultaneously present with the relevant cues, with pitch, timbre and all of the formants co-present. Given listeners' history with this, they may have never developed the need for a buffered approach for vowels, because the relevant context is always available. In contrast, for fricatives, the frication alone is rarely sufficient to identify the talker or the vowel (Lee, Dutton, \& Ram, 2010; McMurray \& Jongman, 2011). As a result, for fricatives, the relevant context cannot be identified during the fricative.

Clearly, context is not strictly necessary (as the gating studies show), so it cannot be that listeners are required wait for context. However, over a lifetime of experience with fricatives listeners learn that they must wait to acquire contextual information since it is not simultaneously available. This could lead to a buffered strategy even in contexts where context is constant (e.g., in these experiments when the talker was the same across the experiment).

Is it even speech? Auditory grouping. Listeners may also wait because at an implicit level, the perceptual system is not sure whether the input is even speech. Fricatives simply do not sound like other speech sounds. They are aperiodic and have a higher frequency than laryngeal voicing, which pervades most of the speech signal. Given this, they may sound quite a bit more like environmental sounds and it may not be until unambiguously speech-like information (the 
vocoid) arrives that the perceptual system bothers activating lexical items. To some extent Experiment 3 rules this out by showing the same integration strategy even when the fricatives are preceded by a sentence which provides clear information that speech is coming.

However, more broadly, if lexical access is preceded by a stage of auditory grouping, such an explanation is still viable. General auditory grouping principles (Bregman, 1990) predict that it may be difficult to integrate information across large frequency bands. Fricatives—unlike most other speech sounds - require such integration. Most information needed for other sounds is below $2000 \mathrm{~Hz}$, whereas perceiving a fricative requires integrating frication at $4000 \mathrm{~Hz}$ or above, with lower frequency $(<2500 \mathrm{hz})$ spectral information. As a result, under a purely auditory grouping account, the frication may not auditorially cohere with the subsequent vocoids.

Challenging this are a number of streaming on fricative-vowel pairs. In these studies, FV syllables are heard as a sequence with two streams indicated by the fact that alternating syllables were spoken by different talkers (David, Lavandier, Grimault, \& Oxenham, 2017b), or at different locations (David, Lavandier, Grimault, \& Oxenham, 2017a). These have generally shown that listeners can group fricatives with their vowels into a single coherent stream. However, prior work suggests that formant transitions are necessary for this (Stachurski, Summers, \& Roberts, 2015) - when transitions are eliminated (flat formants) streaming is reduced. Thus, even as streaming may occur across syllables, within a syllable there may still be a level of grouping or binding that is challenging.

If this auditory grouping account is correct, it offers a novel twist on a common critique of the idea that speech processing cannot be performed via auditory grouping principles (e.g., Remez, Rubin, Berns, Pardo, \& Lang, 1994). This critique argues that auditory grouping principles cannot account for the coherence of the speech stream, because fricatives are higher 
frequency and timbrally distinct from other speech sounds, preventing a single stream.

Classically, this has argued that speech perception must be handled by more speech specific principles. However, our work, suggests that both may be correct: auditory grouping principles may in fact explain speech processing, and this leads to the consequence that fricatives require additional mechanisms to be integrated with the rest of the signal. Under this view, fricatives do not stream with the rest of speech, and this is the source of the difficulty of integrating fricatives and formant transitions.

The Vowel as an Organizing Unit for the Syllable. Finally, speech may be processed using the vowel as the primary organizing unit. Under this account, vowels serve as the anchor for establishing a syllable representation (Hoequist., 1983; Marcus, 1981). Consequently, not until the vowel is identified can the rest of the syllable be constructed. Diehl et al. (1987) present evidence consistent with this, showing that the response time to detect a word initial consonant was affected by the intrinsic duration of the vowel. This could also explain the importance of formant transitions for grouping sequences of fricative-vowel transitions into a stream (Stachurski et al., 2015) - the transitions help bind the fricative to the all important vowel.

Such an account could explain the delays in processing fricatives here: listeners must wait for the vowel to initiate lexical access. In contrast, stops may be processed immediately because they occur shortly before the vowel (less than 50ms) and contain substantial coarticulation which can cue the vowel early (Strange et al., 1983) ${ }^{2}$. A vowel centered account can also explain why Mitterer and Reinisch (2013) observe early effects of frication in word final /s/ vs. /f/ (though these are not strong, see Supplement 6), while we do not, even in medial

\footnotetext{
${ }^{2}$ This may not explain Experiment 4, where listeners could identify fricatives without vowel information. Although again, it is important to point out that being able to do this when forced is not the same as being able to do this under normal circumstances.
} 
position in Experiment 3. In their case, the vowel is heard (initiating lexical access) before the fricative arrives. This enables immediate integration at the onset of the fricative. In contrast, in Experiment 3, while there was a vowel preceding the fricative, it did not belong to the target word so lexical access could not begin until after the fricative.

It may also be the case that cues for stop consonants also cannot be integrated until the arrival of the vocoid - indeed VOT cannot be identified until this point. Given that the VOTs in this study and prior work (McMurray, Clayards, et al., 2008; Toscano \& McMurray, 2012) were quite short $(\sim 40 \mathrm{msec})$, this may be below the temporal resolution of our measures. However, if this is the case, fricatives may not be special at all, but may merely highlight a general fact about cue integration.

Summary. At this point, it remains an open question as to why listeners use a memory buffer strategy for fricatives but not for other speech sounds. However, given the difficulties in a reliability account, and the fact that fricative-vowel syllables can stream (David et al., 2017a, 2017b), the vowel-as-organizer account may offer the most coherent account, particularly given converging evidence for that from other domains (Diehl et al., 1987). However, a definitive test of this awaits. What is clear is that the present experiments offer the first evidence that listeners use a memory buffer integration strategy for at least some sounds.

\section{General implications for language processing}

Substantial evidence suggests a continuous cascade between sub-lexical processes like cue-integration, categorization and talker compensation and higher level lexical processes like lexical access. This work suggests sublexical processing is not encapsulated from downstream processes; rather intermediate stages of sublexical processing immediately influence lexical 
competition. The evidence for immediate integration in stop- consonants and vowels (Reinisch \& Sjerps, 2013; Toscano \& McMurray, 2012, 2015) offers a clear example of this. Lexical activation does not wait for cue-integration to complete; rather the preliminary states (when perhaps only a single cue is available) influence lexical activation immediately. Similarly, within-category differences in phonetic cues like VOT cascade to produce graded lexical activation (Andruski et al., 1994; McMurray, Aslin, et al., 2008; Utman, Blumstein, \& Burton, 2000). This suggests categorization is not complete when lexical access begins. Finally, listeners do not fully compensate for talker variability prior to lexical access (Creel, Aslin, \& Tanenhaus, 2008; Goldinger, 1998) (though see, McLennan \& Luce, 2005). In fact, to our knowledge there is little evidence that any type of speech information is held back from lexical processing.

In the extreme, such findings could support the view that distinct sublexical processing may not be necessary for lexical access. Graded acoustic cues could simply activate words in the lexicon and those words could compete such that phonological categories are not identified prior to lexical access, but emerge out of competition between words. Similarly, partial activation for competitors on the basis of early cues could maintain the likelihood of one category or another while the system waits for more cues, such that cues are integrated lexically, not sublexically. Finally, talker information could immediately activate exemplars in the lexicon that could compete during lexical activation to implicitly abstract over talker.

The results of the current experiments challenge this framing by offering unambiguous evidence for at least one partially encapsulated sublexical process of some kind. There is a clear stage of processing in which perceptual information is being stored, but lexical level processes do not have access to it. Our evidence does not suggest that this is not necessarily a phonemic representation. Rather, the information in the memory buffer is most likely an auditory 
representation, not a phoneme decision, since it appears to be stored before all of the cues for the phoneme are available, and it can be released before the last cue arrives (as in the VOT / VL case). Instead, the most likely explanation is that something about frication spectrum is temporarily stored. It could be a purely echoic memory or memory of cue-values such as the spectral mean. Experiment 6 shows clearly however, that it is the onset of the vocoid that seems to release the information from this buffer. More importantly, is suggests that there are clear auditory processing stages that precede and are independent of lexical access.

More broadly, this work even though it starts from a fairly narrow issue in speech perception- provides some of the first evidence of an encapsulated memory or integration system across language. This speaks to broader debates about whether memory for prior linguistic input is explicitly represented (Caplan \& Waters, 1999; Gathercole \& Baddeley, 1990; Just \& Carpenter, 1992) or emerges implicitly out of competition/activation dynamics (Christiansen \& MacDonald, 2009; Gupta \& Tisdale, 2009). Our data does not suggest the necessity of explicit memory in general. Rather, the robust evidence for immediate integration for other speech cues (McMurray, Clayards, et al., 2008; Reinisch \& Sjerps, 2013; Toscano \& McMurray, 2012) and factors (Kingston et al., 2016; Mitterer \& Reinisch, 2013; Toscano \& McMurray, 2015) raises the possibility of a hybrid model combing both implicit memory processes deriving from activation or recurrence, and explicit memory of some kind. It is also consistent with more recent work (McMurray, Farris-Trimble, \& Rigler, 2017) showing that pre-lingually deaf cohlear implant users may also delay lexical access to accumulate information, potentially as a way to minimize competition.

This work clearly suggests that there may be some specific problems - in this case the perceptual problem of integrating a radically different type of acoustic input-where memory of 
some kind is required. It remains to be seen if analogous problems may also arise at higher

levels of language such as syntactic and semantic processing.

\section{Acknowledgements}

The authors would like to thank Dan McEchron and members of the MACLab for assistance with data collection, Chuck Clifton, Mark Pitt, James McQueen Arty Samuel for valuable comments on an earlier draft, and Michael Tanenhaus for suggesting Experiment 4. Portions of this work were previously reported as the doctoral dissertation of M.E.G. This work was supported by NIH grants, DC000242, and DC008089 awarded to BM.

\section{References}

Allopenna, P., Magnuson, J. S., \& Tanenhaus, M. K. (1998). Tracking the time course of spoken word recognition using eye-movements: evidence for continuous mapping models. Journal of Memory and Language, 38(4), 419-439.

Andruski, J. E., Blumstein, S. E., \& Burton, M. W. (1994). The effect of subphonetic differences on lexical access. Cognition, 52, 163-187.

Apfelbaum, K. S., Blumstein, S. E., \& McMurray, B. (2011). Semantic priming is affected by real-time phonological competition: Evidence for continuous cascading systems. Psychonomic Bulletin and Review, 18(1), 141-149.

Bates, D., \& Sarkar, D. (2011). lme4: Linear mixed-effects models using S4 classes GNU Public License.

Beddor, P. S., Harnsberger, J. D., \& Lindemann, S. (2002). Language-specific patterns of vowelto-vowel coarticulation: acoustic structures and their perceptual correlates. Journal of Phonetics, 30(4), 591-627.

Bharucha, J. J. (1987). Music cognition and perceptual facilitation: A connectionist framework. Music Perception, 5, 1-30.

Bregman, A. S. (1990). Auditory scene analysis: the perceptual organization of sound.

Caplan, D., \& Waters, G. S. (1999). Verbal working memory and sentence comprehension. Behavioral and Brain Sciences, 22(1), 77-94.

Christiansen, M. H., \& MacDonald, M. C. (2009). A Usage-Based Approach to Recursion in Sentence Processing. Language Learning, 59, 126-161. doi:10.1111/j.14679922.2009.00538.x

Cole, J. S., Linebaugh, G., Munson, C., \& McMurray, B. (2010). Unmasking the acoustic effects of vowel-to-vowel coarticulation: A statistical modeling approach. Journal of Phonetics, 38(2), 167-184.

Creel, S., Aslin, R. N., \& Tanenhaus, M. K. (2008). Heeding the voice of experience: The role of talker variation in lexical access. Cognition, 106(2), 633-664.

Dahan, D., \& Magnuson, J. S. (2006). Spoken-word recognition. In M. J. Traxler \& M. A. Gernsbacher (Eds.), Handbook of Psycholinguistics (pp. 249-283). Amsterdam: Academic Press.

Dahan, D., Magnuson, J. S., \& Tanenhaus, M. K. (2001). Time course of frequency effects in 
spoken-word recognition: Evidence from eye movements. Cognitive Psychology, 42, 317-367.

Dahan, D., Magnuson, J. S., Tanenhaus, M. K., \& Hogan, E. (2001). Subcategorical mismatches and the time course of lexical access: Evidence for lexical competition. Language and Cognitive Processes, 16(5/6), 507-534.

Dahan, D., \& Tanenhaus, M. K. (2005). Looking at the rope when looking for the snake: Conceptually mediated eye movements during spoken-word recognition. Psychonomic Bulletin \& Review, 12(3), 453-459.

Daniloff, R., \& Moll, K. (1974). Coarticulation of Lip Rounding. In N. J. Lass (Ed.), Experimental Phonetics (pp. 100-114): MSS Information Corporation.

David, M., Lavandier, M., Grimault, N., \& Oxenham, A. J. (2017a). Discrimination and streaming of speech sounds based on differences in interaural and spectral cues. The Journal of the Acoustical Society of America, 142(3), 1674-1685. doi:10.1121/1.5003809

David, M., Lavandier, M., Grimault, N., \& Oxenham, A. J. (2017b). Sequential stream segregation of voiced and unvoiced speech sounds based on fundamental frequency. Hearing Research, 344, 235-243. doi:https://doi.org/10.1016/j.heares.2016.11.016

Diehl, R. L., Kluender, K. R., Foss, D. J., Parker, E. M., \& Gernsbacher, M. A. (1987). Vowels as islands of reliability. Journal of Memory and Language, 26(5), 564-573. doi:https://doi.org/10.1016/0749-596X(87)90143-4

Dufour, S., Brunellière, A., \& Frauenfelder, U. H. (2013). Tracking the Time Course of WordFrequency Effects in Auditory Word Recognition With Event-Related Potentials. Cognitive Science, 37(3), 489-507. doi:doi:10.1111/cogs.12015

Elman, J. L. (1990). Finding structure in time. Cognitive Science, 14, 179. doi:10.1016/03640213(90)90002-E

Elman, J. L. (2009). On the meaning of words and dinosaur bones: Lexical knowledge without a lexicon. Cognitive Science, 33, 547-582.

Ernst, M. O., \& Banks, M. S. (2002). Humans integrate visual and haptic information in a statistically optimal fashion. Nature, 415, 429-433.

Fedorenko, E., Gibson, E., \& Rohde, D. (2006). The nature of working memory capacity in sentence comprehension: Evidence against domain-specific working memory resources. Journal of Memory and Language, 54(4), 541-553. doi:https://doi.org/10.1016/j.jml.2005.12.006

Forrest, K., Weismer, G., Milenkovic, P., \& Dougall, R. N. (1988). Statistical analysis of wordinitial voiceless obstruents: Preliminary data. Journal of the Acoustical Society of America, 84, 115-124.

Frazier, L. (1987). Sentence processing: A tutorial review.

Frazier, L., \& Rayner, K. (1982). Making and correcting errors during sentence comprehension: Eye movements in the analysis of structurally ambiguous sentences. Cognitive Psychology, 14(2), 178-210.

Galle, M. E. (2014). Integration of multiple and asynchronous acoustic cues to word initial fricatives and context compensation in 7-year olds, 12-year-olds and adults. (Ph.D.), University of Iowa,

Gaskell, M. G., \& Marslen-Wilson, W. D. (1997). Integrating form and meaning: a distributed model of speech perception. Language and Cognitive Processes, 12(5/6), 613-656.

Gathercole, S. E., \& Baddeley, A. D. (1990). Phonological memory deficits in language disordered children: Is there a causal connection? Journal of Memory and Language, 29, 
336-360.

Goldinger, S. D. (1998). Echoes of Echos? An episodic theory of lexical access. Psychological Review, 105, 251-279.

Gupta, P., \& Tisdale, J. (2009). Does phonological short-term memory causally determine vocabulary learning? Toward a computational resolution of the debate. Journal of Memory and Language, 61(4), 481-502.

Hillenbrand, J. M., Clark, M. J., \& Houde, R. A. (2000). Some effects of duration on vowel recognition. Journal of the Acoustical Society of America, 108(6), 3013-3022.

Hoequist., C. E. (1983). The Perceptual Center and Rhythm Categories. Language and Speech, 26(4), 367-376.

Ishida, M., Samuel, A. G., \& Arai, T. (2016). Some people are "More Lexical" than others. Cognition, 151, 68-75. doi:http://dx.doi.org/10.1016/j.cognition.2016.03.008

Jacobs, R. A. (2002). What determines visual cue reliability? Trends in Cognitive Sciences, 6 , 345-350.

Jenkins, J. J., Strange, W., \& Miranda, S. (1994). Vowel identification in mixed-speaker silentcenter syllables. The Journal of the Acoustical Society of America, 95(2), 1030-1043. doi:10.1121/1.410014

Johnson, K. (1991). Differential effects Of Speaker and Vowel Variability on Fricative Perception. Language and Speech, 34(3), 265-279. doi:10.1177/002383099103400304

Jongman, A., Wayland, R., \& Wong, S. (2000). Acoustic characteristics of English fricatives. Journal of the Acoustical Society of America, 106, 1252-1263.

Just, M. A., \& Carpenter, P. A. (1992). A capacity theory of comprehension: Individual differences in working memory. Psychological Review, 99(1), 122-149.

King, J., \& Just, M. A. (1991). Individual differences in syntactic processing: The role of working memory. Journal of Memory and Language, 30(5), 580-602.

Kingston, J., Levy, J., Rysling, A., \& Staub, A. (2016). Eye Movement Evidence for an Immediate Ganong Effect. Journal of Experimental Psychology: Human Perception and Performance, 42(12), 1969-1988.

Lee, C. Y., Dutton, L., \& Ram, G. (2010). The role of speaker gender identification in F0 height estimation from multispeaker, brief speech segments. Journal of the Acoustical Society of America, 128, 384-388.

Levy, R., Bicknell, K., Slattery, T., \& Rayner, K. (2009). Eye movement evidence that readers maintain and act on uncertainty about past linguistic input. Proceedings of the National Academy of Sciences, 106(50), 21086-21090.

Lisker, L. (1986). "Voicing" in English: a catalogue of acoustic features signaling /b/ versus /p/ in trochees. Language and Speech, 29(1), 3-11.

Lisker, L., \& Abramson, A. S. (1964). A cross-language study of voicing in initial stops: acoustical measurements. Word, 20, 384-422.

MacDonald, M. C., Pearlmutter, N. J., \& Seidenberg, M. S. (1994). Lexical nature of syntactic ambiguity resolution. Psychological Review, 101, 676-703.

Mann, V. A., \& Repp, B. (1980). Influence of the vocalic context on the ? - s distinction. Perception \& Psychophysics, 28(3), 213-228.

Marcus, S. M. (1981). Acoustic determinants of perceptual center (P-center) location. Perception \& Psychophysics, 30(3), 247-256.

Marslen-Wilson, W. D. (1987). Functional parallelism in spoken word recognition. Cognition, 25(1-2), 71-102. 
Marslen-Wilson, W. D., \& Tyler, L. K. (1980). The temporal structure of spoken language understanding. Cognition, 8(1), 1-71.

Marslen-Wilson, W. D., \& Zwitserlood, P. (1989). Accessing spoken words: The importance of word onsets. Journal of Experimental Psychology: Human Perception and Performance, 15, 576-585.

Matuschek, H., Kliegl, R., Vasishth, S., Baayen, H., \& Bates, D. (2017). Balancing Type I error and power in linear mixed models. Journal of Memory and Language, 94, 305-315.

McClelland, J. L., \& Elman, J. L. (1986). The TRACE model of speech perception. Cognitive Psychology, 18(1), 1-86.

McLennan, C., \& Luce, P. A. (2005). Examining the Time Course of Indexical Specificity Effects in Spoken Word Recognition. Journal of Experimental Psychology: Learning, Memory and Cognition, 31(2), 306-321.

McMurray, B., Aslin, R. N., Tanenhaus, M. K., Spivey, M. J., \& Subik, D. (2008). Gradient sensitivity to within-category variation in words and syllables. Journal of Experimental Psychology, Human Perception and Performance, 34(6), 1609-1631.

McMurray, B., Clayards, M., Tanenhaus, M. K., \& Aslin, R. N. (2008). Tracking the time course of phonetic cue integration during spoken word recognition. Psychonomic Bulletin and Review, 15(6), 1064-1071.

McMurray, B., Farris-Trimble, A., \& Rigler, H. (2017). Waiting for lexical access: Cochlear implants or severely degraded input lead listeners to process speech less incrementally. Cognition, 169, 147-164.

McMurray, B., Farris-Trimble, A., Seedorff, M., \& Rigler, H. (2016). The effect of residual acoustic hearing and adaptation to uncertainty in Cochlear Implant users. Ear and Hearing, 37(1), 37-51.

McMurray, B., \& Jongman, A. (2011). What information is necessary for speech categorization? Harnessing variability in the speech signal by integrating cues computed relative to expectations. Psychological Review, 118(2), 219-246.

McMurray, B., Samelson, V. S., Lee, S. H., \& Tomblin, J. B. (2010). Individual differences in online spoken word recognition: Implications for SLI. Cognitive Psychology, 60(1), 1-39.

McMurray, B., Tanenhaus, M. K., \& Aslin, R. N. (2002). Gradient effects of within-category phonetic variation on lexical access. Cognition, 86(2), B33-B42.

McMurray, B., Tanenhaus, M. K., \& Aslin, R. N. (2009). Within-category VOT affects recovery from "lexical" garden paths: Evidence against phoneme-level inhibition. Journal of Memory and Language, 60(1), 65-91.

McRae, K., Spivey-Knowlton, M. J., \& Tanenhaus, M. K. (1998). Modeling the influence of thematic fit (and other constraints) in on-line sentence comprehension. Journal of Memory and Language, 38, 283-312.

Miller, J. L., \& Dexter, E. R. (1988). Effects of speaking rate and lexical status on phonetic perception. Journal of Experimental Psychology: Human Perception and Performance, 14(3), 369.

Miller, J. L., \& Volaitis, L. E. (1989). Effect of speaking rate on the perceptual structure of a phonetic category. Perception \& Psychophysics, 46(6), 505-512.

Miller, J. L., \& Wayland, S. (1993). Limits on the limitations of context-conditioned effects in the perception of [b] and [w]. Perception and Psychophysics, 54, 205-210.

Miller, J. O., Patterson, T., \& Ulrich, R. (1998). Jackknife-based method for measuring LRP onset latency differences. Psychophysiology, 35, 99-115. 
Mitterer, H., \& Reinisch, E. (2013). No delays in application of perceptual learning in speech recognition: Evidence from eye tracking. Journal of Memory and Language, 69(4), 527545.

Nearey, T. M. (1997). Speech perception as pattern recognition. Journal of the Acoustical Society of America, 101(6), 3241-3254.

Norris, D., \& McQueen, J. M. (2008). Shortlist B: A Bayesian model of continuous speech recognition. Psychological Review, 115(2), 357-395.

Oden, G., \& Massaro, D. W. (1978). Integration of featural information in speech perception. Psychological Review, 85(3), 172-191.

Peterson, G. E., \& Barney, H. L. (1952). Control methods used in a study of the vowels. Journal of the Acoustical Society of America, 24, 175-184.

Reinisch, E., \& Sjerps, M. J. (2013). The uptake of spectral and temporal cues in vowel perception is rapidly influenced by context. Journal of Phonetics, 41(2), 101-116.

Remez, R. E., Rubin, P. E., Berns, S. M., Pardo, J. S., \& Lang, J. M. (1994). On the perceptual organization of speech. Psychological Review, 101(1), 129-156.

Repp, B. H. (1981). Perceptual equivalence of two kinds of ambiguous speech stimuli. Bulletin of the Psychonomic Society, 18(1), 12-14. doi:10.3758/bf03333556

Shinn, P. C., Blumstein, S. E., \& Jongman, A. (1985). Limits of context conditioned effects in the perception of [b] and [w]. Perception and Psychophysics, 38(5), 397-407.

Sjerps, M. J., McQueen, J. M., \& Mitterer, H. (2013). Evidence for precategorical extrinsic vowel normalization. Attention, Perception, \& Psychophysics, 75(3), 576-587. doi:10.3758/s13414-012-0408-7

Smits, R., Warner, N., McQueen, J. M., \& Cutler, A. (2003). Unfolding of phonetic information over time: a database of Dutch diphone perception. J Acoust Soc Am, 113(1), 563-574.

Stachurski, M., Summers, R. J., \& Roberts, B. (2015). The verbal transformation effect and the perceptual organization of speech: influence of formant transitions and F0-contour continuity. Hearing Research, 323, 22-31.

Strange, W., Jenkins, J. J., \& Johnson, T. L. (1983). Dynamic specification of coarticulated vowels. The Journal of the Acoustical Society of America, 74(3), 695-705. doi:doi:http://dx.doi.org/10.1121/1.389855

Summerfield, Q. (1981). Articulatory rate and perceptual constancy in phonetic perception. Journal of the Acoustical Society of America, 7(5), 1074-1095.

Tanenhaus, M. K., Spivey-Knowlton, M. J., Eberhard, K. M., \& Sedivy, J. C. (1995). Integration of visual and linguistic information in spoken language comprehension. Science, 268, 1632-1634.

Tanenhaus, M. K., \& Trueswell, J. C. (1995). Sentence Comprehension. In P. D. Eimas \& J. L. Miller (Eds.), Handbook in perception and Cognition. Speech, language and communication. (Vol. 11, pp. 217-262). New York, NY: Academic Press.

Toscano, J. C., \& McMurray, B. (2010). Cue integration with categories: Weighting acoustic cues in speech using unsupervised learning and distributional statistics. Cognitive Science, 34(3), 436-464.

Toscano, J. C., \& McMurray, B. (2012). Online integration of acoustic cues to voicing: Natural vs. synthetic speech. Attention, Perception \& Psychophysics, 74(6), 1284-1301.

Toscano, J. C., \& McMurray, B. (2015). The time-course of speaking rate compensation: Effects of sentential rate and vowel length on voicing judgments. Language, Cognition and Neuroscience, 30, 529-543. 
Tyler, L. K., \& Wessels, J. (1985). Is gating an on-line task? Evidence from naming latency data. Perception \& Psychophysics, 38(3), 217-222. doi:10.3758/bf03207148

Utman, J. A. (1998). Effects of local speaking rate context on the perception of voice-onset time in initial stop consonants. J Acoust Soc Am, 103(3), 1640-1653.

Utman, J. A., Blumstein, S. E., \& Burton, M. W. (2000). Effects of subphonetic and syllable structure variation on word recognition. Perception \& Psychophysics, 62(6), 1297-1311.

van den Brink, D., Brown, C. M., \& Hagoort, P. (2006). The Cascaded Nature of Lexical Selection and Integration in Auditory Sentence Processing. Journal of Experimental Psychology: Learning, Memory \& Cognition, 32(3), 364-372.

Warner, N., McQueen, J. M., \& Cutler, A. (2014). Tracking perception of the sounds of English. $J$ Acoust Soc Am, 135(5), 2995-3006. doi:10.1121/1.4870486

Weber, A., \& Scharenborg, O. (2012). Models of spoken-word recognition. Wiley Interdisciplinary Reviews: Cognitive Science, 3(3), 387-401. doi:10.1002/wcs.1178

Yee, E., \& Sedivy, J. C. (2006). Eye movements to pictures reveal transient semantic activation during spoken word recognition. Journal of Experimental Psychology: Learning, Memory, and Cognition, 32, 1-14.

Zhang, X., \& Samuel, A. G. (2018). Is speech recognition automatic? Lexical competition, but not initial lexical access, requires cognitive resources. Journal of Memory and Language, 100, 32-50. doi:https://doi.org/10.1016/j.jml.2018.01.002

Zwitserlood, P. (1989). The locus of the effects of sentential-semantic context in spoken-word processing. Cognition, 32, 25-64. 


\title{
Online Supplement to.
}

\section{What are you waiting for?}

Real-time integration of cues for fricatives suggests encapsulated auditory memory.

\author{
Marcus E. Galle \\ Jamie Klein-Packard \\ Dept. of Psychological and Brain Sciences \\ University of Iowa \\ Kayleen Schreiber \\ Interdisciplinary Program in Neuroscience \\ University of Iowa \\ and \\ Bob McMurray \\ Dept. of Psychological and Brain Sciences \\ Dept. of Communication Sciences and Disorders \\ Dept. of Linguistics \\ University of Iowa
}

\author{
Corresponding Author \\ Bob McMurray \\ E11 SSH \\ Dept. of Psychological and Brain Sciences \\ University of Iowa \\ Iowa City, IA 52242 \\ 319-335-2408 \\ Bob-mcmurray@uiowa.edu
}




\section{S1. Visualization of Stimulus Construction for Fricatives}

A.

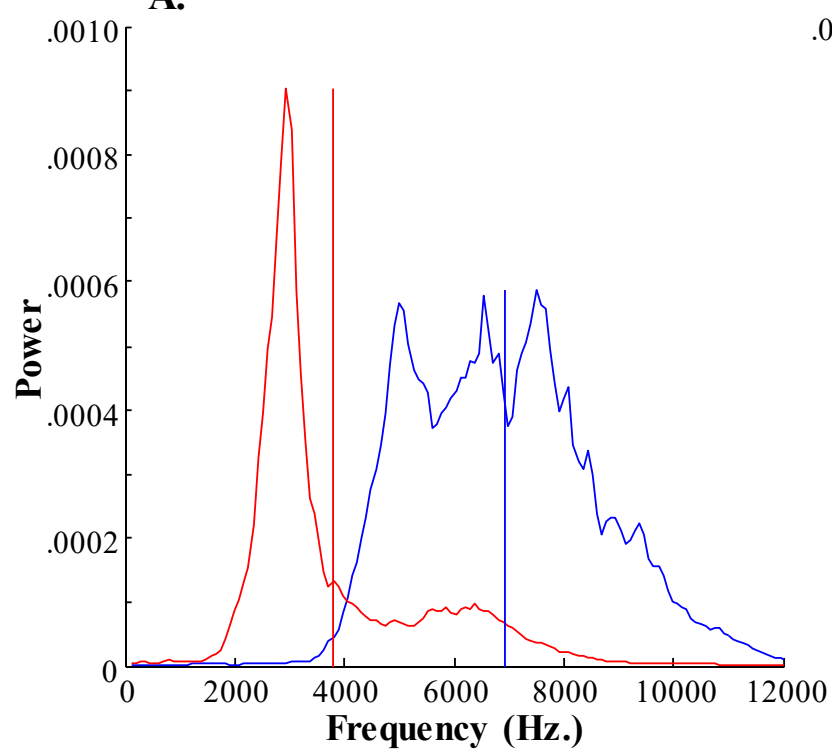

C.

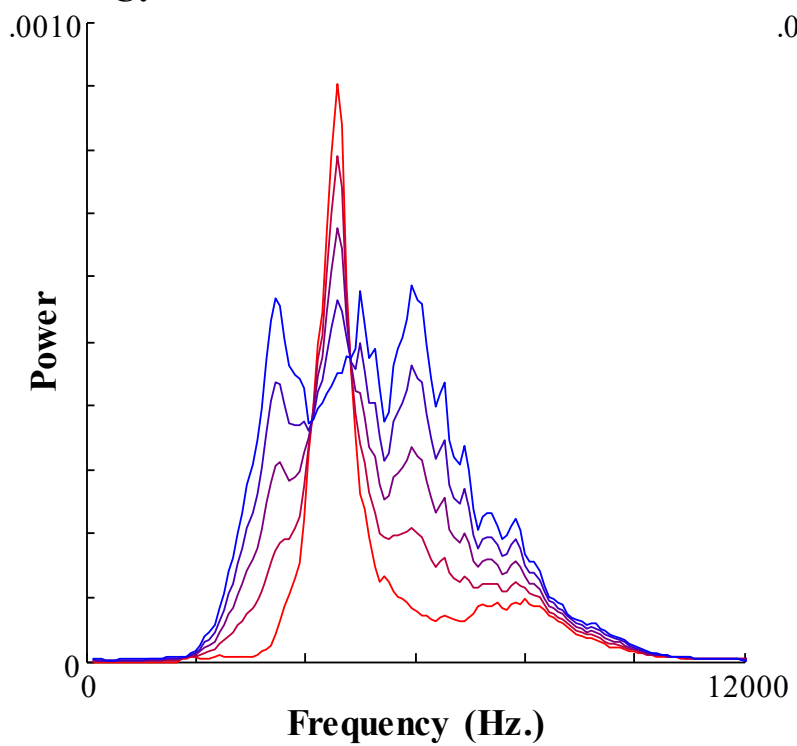

B.

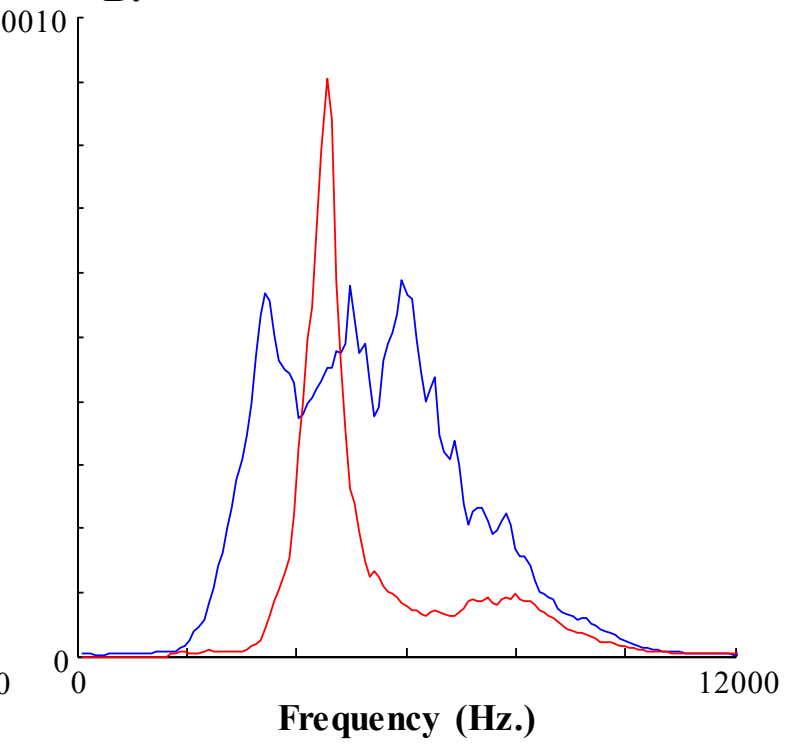

D.

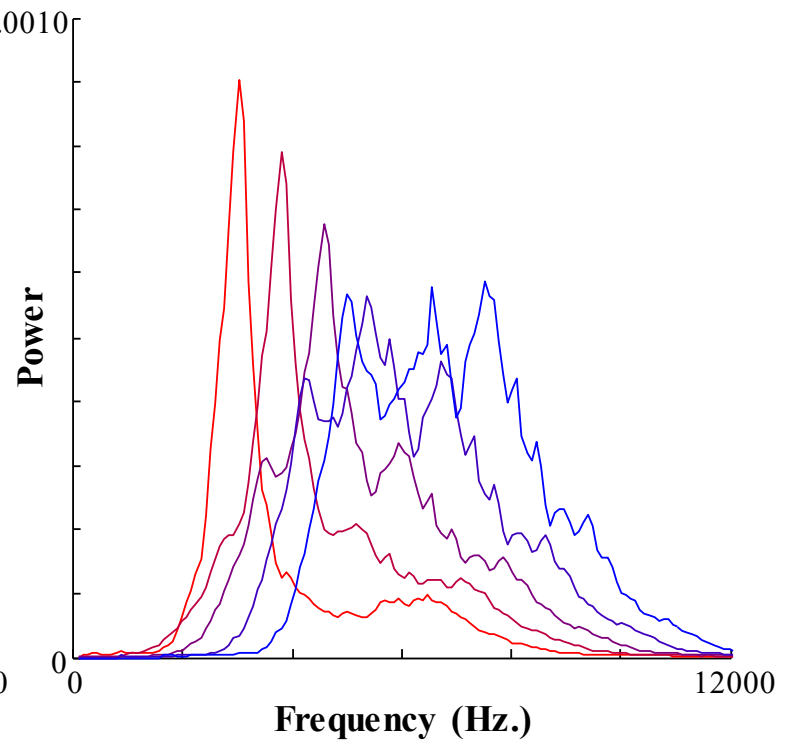

Figure S1: Stimulus construction methods for fricatives. A) The Long Term Average Spectrum (LTAS) for each fricative (red $=/ \mathrm{f} /$, blue $=/ \mathrm{s} /$ ) is extracted and the spectral mean (vertical bars) is computed. B) LTAS's for each fricative are shifted in frequency space such that the spectral means align. C) LTAS's are averaged to create 7 steps, still centered at the same peak frequency. D) The new spectra are shifted in frequency space in 7 even steps from the original spectral mean of the $/ \mathrm{J} /$ to the original spectral mean of the $/ \mathrm{s} /$.

Two additional steps are not shown.

Prior to (A), we computed LTAS' for multiple exemplars of $/ \mathrm{s} /$ and $/ \mathrm{f} /$, shifted them in frequency space to have the same spectral mean (which was the average of all the spectral means) and 
averaged the LTAS's to create a prototypical/s/ and / $/$ LTAS. These are what are shown as the two LTAS in panel A.

After (D), we filtered white noise using each LTAS to create the continua steps. We then imposed an amplitude envelope on the noise corresponding to the average of all of the envelopes. 


\section{S2. Alternative analysis of the onset of effects (Experiment 1)}

Kingston, Levy, Rysling, and Staub (2016) show data that looks like the immediate utilization of frication spectrum during the frication. They analyzed their data in sequential 100 msec bins. They report that during their first time bin (200 msec to $300 \mathrm{msec} ; 0-100 \mathrm{msec}$ after fricative onset if one saccount for the $200 \mathrm{msec}$ oculomotor delay) there was a significant difference in fixations as a function of the fricative continuum step. It is important to note that this was a small effect (that may not have been significant if family-wise error was accounted for), and that the effect grows much larger at later time windows. However, due to the fact that their fricatives were short ( $150 \mathrm{msec}$, compared to $290 \mathrm{msec}$ for ours), these later time windows also include the vocoid. Thus, only one data point really supports the claim of immediate utilization of frication.

However, this study was not intended to look at multiple cue integration (they did not manipulate a second acoustic cue), so the use of frication cannot be compared to anything else directly. Thus, their results do not offer an explicit test of the immediate integration vs. buffered accounts. However, to the extent that a buffered approach (which our data support) does not predict utilization of the frication spectrum before the arrival of the vocoid, that one data point would appear problematic for our account.

In the main text and Supplement S6, we discuss several task-related issues that may give rise to the differences between their study and ours: the use of letters as stimuli (which was reasonable given their goals), and the use of sample averaged fricatives. Here we consider another difference: the analytic strategy. Our hypotheses were evaluated by comparing the onset times of each effect, as most prior studies of asynchronous cue integration have done (McMurray, Clayards, Tanenhaus, \& Aslin, 2008; Reinisch \& Sjerps, 2013; Toscano \& McMurray, 2012, 2015). In contrast, Kingston et al. (2016) assessed the magnitude of each effect at each time bin and compared it to 0 . This is perhaps not ideal as this assessment will be sensitive to both the mean and variance of the effect - weaker effects which have more variance could appear later simply because they are higher variance, not because of a difference in the mean. Nonetheless, we wanted to evaluate this analytic strategy to ask if we would find evidence for immediate integration under this approach.

\section{Methods}

We examined the data from Experiment 1 using a variant of Kingston et al.,'s (2016) procedure. Experiment 1 manipulated three factors within subject: frication spectrum (6 step continuum), formant transition (/ $/ \mathrm{V} / \mathrm{vs}$. $/ \mathrm{s} /$ appropriate transitions) and vowel rounding (rounded vs. unrounded). There were 8 continua ( 4 rounded, 4 unrounded).

To conduct our analysis we started with our primary measure, biass-s. The average biass-s was computed in $100 \mathrm{msec}$ time bins at each frication step, transition, rounding and continuum. Time windows started at $150 \mathrm{msec}(150,250,350 \ldots)$. This was done because the fricative/vocoid juncture occurred at $450 \mathrm{msec}$. Consequently, if we account for the $200 \mathrm{msec}$ it takes to plan an eye-movement, time windows 100-600 can be assumed to reflect processing that is solely based on the frication; timewindows after that reflect the frication and the vocoid.

At each timewindow we constructed a linear mixed effects model examining all three factors. Fixed effects included step (centered), rounded $(+/-.5$, rounded $=.5)$, transition $(+/-.5, / \mathrm{s} /$ transition $=.5$ ) and all interactions. Models had random intercepts of continuum (e.g., sip/ship, sore/shore) and subject, along with random slopes of step, transition and rounding on subject. Due to difficulties in getting this model to converge at the early time windows, we included no covariance terms. The final model (in R syntax is) is in (1) 


$$
\begin{aligned}
\text { Bias } ~ & \text { Step*round*trans } \\
& +(1 \mid \text { Subject })+(0+\text { Step } \mid \text { Subject })+(0+\text { round } \mid \text { Subject }) \\
& +(0+\text { trans } \mid \text { Subject })+(1 \mid \text { Continua })
\end{aligned}
$$

At each time-step, we saved the coefficients, standard errors and so forth. No attempt was made to correct for family-wise error (similarly to Kingston et al., 2016); however, we point out that such a correction would work in favor of a buffered hypothesis as it would likely delay the detected onset of effects until later in the timecourse when the fixations are more stable and less variable.

\section{Results and Discussion}

The results of each model are shown in Table S1, and Figure S2 shows the timecourse of the effect estimates (unstandardized coefficients, B). These models did not show a significant effect of frication until the 550-650 msec time window. This window is the final time window within the frication, so it appears that some information was available prior to the vocoid. However, this is not equivalent to immediate utilization of the frication. Four time windows were tested after the frication but before the vocoid, (250-350, 350- 450, 450-550, and 550-650), and the first three were /non-significant. Moreover, even at the final bin, this is actually very little use of frication - the absolute magnitude of the effect $(\mathrm{B}=.016)$ is less than $5 \%$ of the value at $1500 \mathrm{msec}(\mathrm{B}=.366)$, suggesting this is rather minimal use of frication (if at all).

The effect of transition and rounding were observed sometime after the frication at 800 msec and $900 \mathrm{msec}$ respectively. However, this should not be interpreted as these effects being significantly later - as Figure S1 indicates all three effects were fairly close in time (and late). Rather these differences are more likely to derive from the much larger variance in the transition and rounding effects (which were weaker and more variable across subjects).

Thus, we do not observe strong evidence for immediate utilization of frication. There was clearly no effect during the first $200 \mathrm{msec}$ of the frication, and only a small effect during the last time bin of the frication.

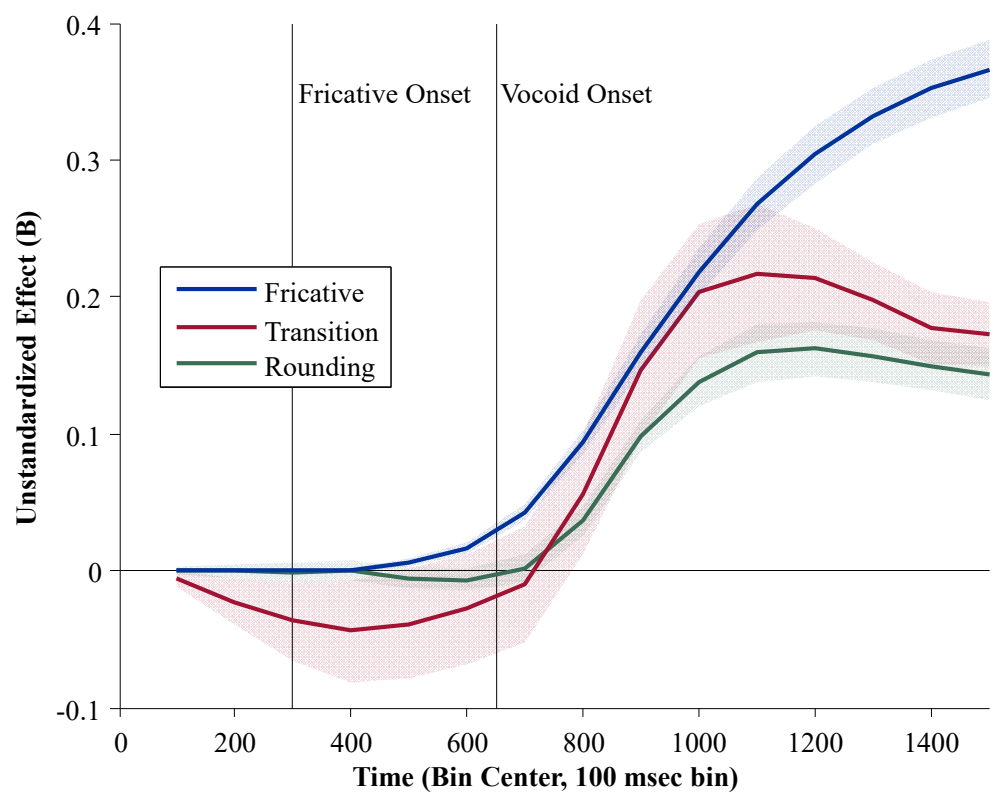

Figure S2: Coefficients of each effect as a function of time. Shaded regions represent SE of the coefficient. 
Table S1. Results of a series of mixed models examining bias as a function of frication step, transition and vowel rounding at consecutive $100 \mathrm{msec}$ time windows. Time is $100 \mathrm{msec}$ bin. The $\mathrm{F} / \mathrm{V}$ refers to what signal was present at that bin (adjusting for the 200 msec oculomotor delay); $\mathrm{F}=$ frication; $\mathrm{V}=$ vocoid. $\mathrm{p}<.2$ is not shown, highlighted rows are significant.

\begin{tabular}{|c|c|c|c|c|c|c|c|c|c|c|c|c|c|c|c|c|}
\hline \multirow{2}{*}{\multicolumn{2}{|c|}{ Time }} & \multicolumn{5}{|c|}{ Step } & \multicolumn{5}{|c|}{ Transition } & \multicolumn{5}{|c|}{ Vowel Rounding } \\
\hline & & B & SE & df & $\mathbf{T}$ & $\mathbf{p}$ & B & SE & df & $\mathbf{T}$ & $\mathbf{p}$ & B & SE & df & $\mathbf{T}$ & $\mathbf{p}$ \\
\hline $50-150$ & - & 0.000 & 0.001 & 23.2 & 0.0 & & 0.000 & 0.003 & 23.1 & 0.2 & & -0.006 & 0.006 & 10.3 & 1.0 & \\
\hline $150-250$ & - & 0.000 & 0.001 & 2197.8 & 0.2 & & 0.000 & 0.005 & 23.1 & 0.1 & & -0.023 & 0.017 & 8.0 & 1.3 & \\
\hline $250-350$ & $\mathrm{~F}$ & 0.000 & 0.002 & 2197.5 & -0.3 & & -0.001 & 0.007 & 23.1 & 0.1 & & -0.037 & 0.028 & 7.3 & 1.3 & \\
\hline $350-450$ & $\mathrm{~F}$ & 0.000 & 0.002 & 2220.4 & -0.1 & & 0.000 & 0.007 & 2220.1 & 0.0 & & -0.044 & 0.037 & 7.1 & 1.2 & \\
\hline $450-550$ & $\mathrm{~F}$ & 0.006 & 0.003 & 23.1 & 1.9 & 0.075 & -0.005 & 0.008 & 2197.1 & 0.6 & & -0.039 & 0.040 & 7.2 & 1.0 & \\
\hline $550-650$ & $\mathrm{~F}$ & 0.016 & 0.004 & 23.1 & 4.1 & 0.00045 & -0.007 & 0.008 & 2197.0 & 0.8 & & -0.028 & 0.040 & 6.6 & 0.7 & \\
\hline $650-750$ & $\mathrm{~V}$ & 0.042 & 0.006 & 23.1 & 7.5 & $<.0001$ & 0.002 & 0.010 & 2197.0 & 0.2 & & -0.010 & 0.042 & 6.9 & 0.2 & \\
\hline $750-850$ & $\mathrm{~V}$ & 0.094 & 0.009 & 23.0 & 10.1 & $<.0001$ & 0.036 & 0.011 & 2197.2 & 3.2 & 0.0012 & 0.056 & 0.044 & 8.0 & 1.3 & \\
\hline $850-950$ & $\mathrm{~V}$ & 0.160 & 0.013 & 23.0 & 12.1 & $<.0001$ & 0.098 & 0.012 & 23.1 & 8.0 & $<.0001$ & 0.147 & 0.051 & 10.2 & 2.9 & 0.016 \\
\hline $9500-1050$ & $\mathrm{~V}$ & 0.218 & 0.017 & 23.0 & 13.2 & $<.0001$ & 0.138 & 0.018 & 23.0 & 7.7 & $<.0001$ & 0.204 & 0.049 & 10.7 & 4.2 & 0.0016 \\
\hline $1050-1150$ & $\mathrm{~V}$ & 0.267 & 0.019 & 23.0 & 14.2 & $<.0001$ & 0.159 & 0.021 & 23.0 & 7.7 & $<.0001$ & 0.217 & 0.050 & 10.6 & 4.4 & 0.0012 \\
\hline $1150-1250$ & $\mathrm{~V}$ & 0.304 & 0.021 & 23.0 & 14.8 & $<.0001$ & 0.162 & 0.020 & 23.0 & 8.0 & $<.0001$ & 0.213 & 0.037 & 11.2 & 5.7 & 0.00012 \\
\hline $1250-1350$ & $\mathrm{~V}$ & 0.332 & 0.021 & 23.0 & 15.5 & $<.0001$ & 0.157 & 0.020 & 23.0 & 8.0 & $<.0001$ & 0.197 & 0.028 & 11.5 & 7.0 & $<.0001$ \\
\hline $1350-1450$ & $\mathrm{~V}$ & 0.352 & 0.021 & 23.0 & 16.5 & $<.0001$ & 0.150 & 0.019 & 23.0 & 7.8 & $<.0001$ & 0.177 & 0.026 & 10.5 & 6.7 & $<.0001$ \\
\hline $1450-1550$ & $\mathrm{~V}$ & 0.366 & 0.021 & 23.0 & 17.8 & $<.0001$ & 0.143 & 0.019 & 23.0 & 7.7 & $<.0001$ & 0.173 & 0.023 & 9.3 & 7.5 & $<.0001$ \\
\hline
\end{tabular}


To put this in perspective, Figure S.3 shows the bias $_{s-f}$ as a function of frication step and time for several timebins. Time window 600 is highlighted in red. It suggests that while there may have been some statistical evidence for late utilization of frication during this time window, this effect is extremely small numerically, and it is not until after the vocoid (e.g., the much larger changes between 700 and $800 \mathrm{msec}$ ) that we see any substantive use of frication. In fact, given that manipulating the length of the fricative (in Experiment 5) actually delays the use of the frication, this would seem to suggest that the onset of the vocoid is pivotal for engaging the use of the much earlier frication.

Given this, there is no evidence in our study for immediate utilization of frication and the only evidence for its use prior to the vocoid is small and weak. Any differences then between our study and that of Kingston et al. (2016) more likely derive from methodological rather than statistical factors.

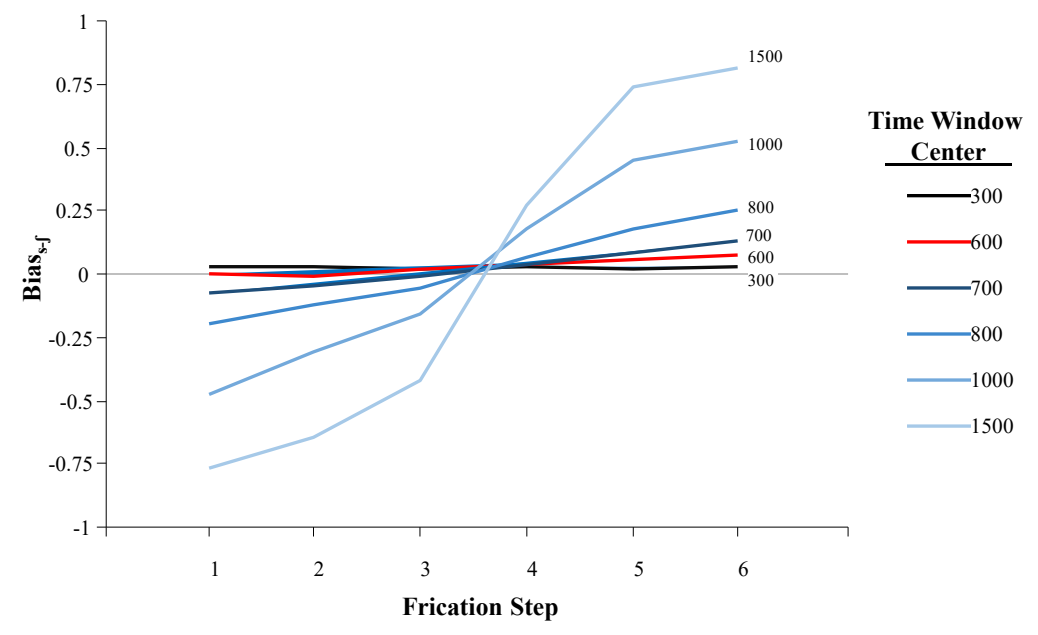

Figure S3. Effect of frication step on Bias s- $_{\text {in }}$ in selected time bins. Time window $600(550-650 \mathrm{msec})$ is highlighted in red as it was the last window prior to the onset of vocoid (blue curves) 


\section{S3. Evidence of effects in eye-movement data for Experiments 2, 3, and 6.}

As in Experiment 1, Experiments 2, 3 and 6 measured when various acoustic cues affected lexical activation to disentangle immediate integration from memory buffer accounts. While our goal was to examine fricative continua, we also tested stop voicing continua to replicate earlier work (McMurray, Clayards, et al., 2008; Toscano \& McMurray, 2012, 2015). The primary analyses in this study concerned when each cue impacted lexical competition. However, as a precursor to that, we evaluated whether each cue affected lexical activation at all. These analyses were reported for Experiment 1 in the main text. Here we report analogous analyses for Experiments 2, 3 and 6. There were no such analysis for Experiment 4 (the gating study, which measured only mouse-clicking) and Experiment 5 used only un-manipulated words and did not manipulate individual cues.

\section{Experiment 2}

In Experiment 2, participants heard fricative stimuli that differed within-subjects by frication (a six-step continuum) and transition (/// vs. /s/). Stimuli differed between-subjects by vowel rounding (round or unrounded). Filler trials included a VOT $\times$ vowel length $(\mathrm{VL})$ continuum.

Fricative cues. We calculated the dependent variable, biass-f, by subtracting the proportion of fixations to the $/ \mathrm{S} /$ objects from the proportion of fixations to the $/ \mathrm{s} /$ objects every 4 msec over the course of each trial (see Figure 3, main text). We observed the most robust lexical activation (e.g., looks to the fricatives were greater than to the control stops) between $600 \mathrm{~ms}$ and $1600 \mathrm{~ms}$, so we used this timeframe to calculate the average biass-s. We used a linear mixedeffects model to determine the effect of frication step, transition and rounding on average biass-f. We started with a base model that included all of the fixed effects and random intercepts for subject and word pair. Adding a random slope of frication step on subject significantly increased fit $\left(\chi^{2}(2)=194.47, p<.001\right)$ and adding a random slope of frication step on word pair, also significantly increased fit $\left(\chi^{2}(2)=64.45, \mathrm{p}<.001\right)$. Adding random slope of transition and its interaction with frication step on subject increased fit $\left(\chi^{2}(7)=16.94, p<.005\right)$, however this same set of terms as a slope on word pair did not increase fit $\left(\chi^{2}(7)=10.33, p=0.17\right)$. More complex models failed to converge. Our final model used frication, transition and rounding and their interactions as fixed effects, with random slopes of frication step and transition on subject and frication on word pair (2).

$$
\text { Bias } \sim \text { Step*round*trans }+(\text { Step*trans } \mid \text { Subject })+(\text { step } \mid \text { wordpair })
$$

Table S.2 shows significant main effects of frication $(\mathrm{p}<.001)$, transition $(\mathrm{p}<.001)$, and rounding $(\mathrm{p}=0.048)$. There was a greater bias toward $/ \mathrm{s} /$ at higher steps, more $/ \mathrm{s} /$ bias toward $/ \mathrm{s} /$ transitions, and more $/ \mathrm{s} /$ bias for rounded vowels. There were no interactions. This validates our investigation of the timing of these cues.

Stop Consonant Voicing. The stop-consonant stimuli for Experiment 2 differed by both vowel length (VL; long vs. short), and voice onset time (VOT; six-step continuum), withinsubject. We subtracted looks to the $/ \mathrm{b} /$ item from looks to the $/ \mathrm{p} /$ item to create the dependent variable, bias $_{p-b}$, and computed the average of this between $600 \mathrm{~ms}$ and $1600 \mathrm{~ms}$ as we did for

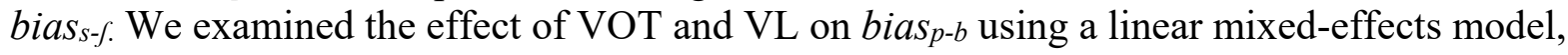
starting with both of the fixed effects and random intercepts for subject and word pair. Adding random slope of VOT on subject significantly increased fit $\left(\chi^{2}(2)=203.62, p<.001\right)$, as did adding random slope of VOT on word pair $\left(\chi^{2}(1)=31.04, p<.001\right)$.. No further gains in model fit 
Table S2. Evidence of effects on biass-f, and biasp-b, in Experiment 2. p>.1 not shown.

\begin{tabular}{|c|c|c|c|c|c|c|}
\hline & Factor & B & SE & df & t & $\mathbf{p}$ \\
\hline \multirow{7}{*}{$\begin{array}{l}\text { Fricatives } \\
\left(\text { bias }_{S-f)}\right.\end{array}$} & Frication & 0.22 & 0.02 & 16.89 & 11.56 & $<0.001$ \\
\hline & Transition & 0.10 & 0.02 & 37.47 & 5.86 & $<0.001$ \\
\hline & Rounding & 0.13 & 0.03 & 8.48 & 2.31 & 0.05 \\
\hline & Frication $\mathrm{x}$ transition & 0.00 & 0.00 & 177.02 & -0.49 & \\
\hline & Frication $\mathrm{x}$ rounding & -0.03 & 0.04 & 16.89 & -0.67 & \\
\hline & Transition $\mathrm{x}$ rounding & 0.03 & 0.03 & 37.47 & 0.80 & \\
\hline & Frication $\mathrm{x}$ trans $\mathrm{x}$ round & -0.02 & 0.02 & 177.02 & -1.59 & \\
\hline \multirow{3}{*}{$\begin{array}{c}\text { Stop- } \\
\text { consonants } \\
\text { (bias } \text { bib }_{p} \text { ) }\end{array}$} & VOT & 0.00 & 0.00 & 0.00 & -12.27 & $<0.001$ \\
\hline & $\mathrm{VL}$ & 0.00 & 0.00 & 0.00 & 6.40 & $<0.001$ \\
\hline & $\mathrm{VOT} \times \mathrm{VL}$ & 0.00 & 0.00 & 0.06 & 0.06 & \\
\hline
\end{tabular}

were seen when we added a random slope of VL and its interaction with VOT on subject $\left(\chi^{2}(7)=\right.$ $5.58, \mathrm{p}=0.59)$ or word pair $\left(\chi^{2}(7)=1.55, \mathrm{p}=0.98\right)$. Therefore, our final model used length and step as fixed effects, with random slopes of step on subject and word pair (3).

$$
\text { Bias } \sim \text { VOT*VL }+(\text { VOT } \mid \text { subject })+(\text { VOT } \mid \text { wordpair })
$$

The results are shown in Table S.2. There were significant main effects of both VOT $(p<0.001)$ and VL $(p<.001)$ wherein long VOTs and short vowels were associated with a greater bias toward $/ \mathrm{p} /$. These findings validate that the two stop-consonant cues also affected overall looking in the predicted direction.

\section{Experiment 3}

Experiment 3 examined the effect of frication, transition and rounding when the fricatives were in semantically neutral sentential contexts. This added a fourth independent factor: whether the sentence contained coarticulation predicting an $/ \mathrm{g} /$ or $/ \mathrm{s} /$. VOT was not manipulated in this experiment so we only analyzed the effects on fricative bias.

Fricative cues. The dependent measure in this model was again bias $_{s-f}$ between 600 and $1600 \mathrm{msec}$. We assessed effect of frication step, transition, rounding, and sentential transition. We started with a base model that included all of the fixed effects and random intercepts for subject and continua. We significantly increased fit by adding random slope of frication step on subject $\left(\chi^{2}(2)=273.41, \mathrm{p}<0.001\right)$ and adding random slope of frication step on word pair $\left(\chi^{2}(2)=\right.$ $110.88, \mathrm{p}<0.001)$. Adding random slope of transition on subject $\left(\chi^{2}(7)=58.91, \mathrm{p}<0.001\right)$, and on word pair $\left(\chi^{2}(7)=51.14, p<0.001\right)$ also increased fit, but more complex models failed to converge. Thus, the final model included frication, transition, rounding, and sentential transition as fixed effects, with random slopes of frication and transition on subject, frication and transition on word pair (4).

$$
\begin{aligned}
\text { Bias } \sim & \text { Step*Transition*Rounding*SentTran }+ \\
& (\text { Step*Transition } \mid \text { Subject })+(\text { Step*Transition } \mid \text { Word Pair })
\end{aligned}
$$

The results are shown in Table S3. As in Experiments 1 and 2, there were significant main effects of frication $(\mathrm{p}<.001)$ and transition $(\mathrm{p}=0.003)$, with greater bias towards $/ \mathrm{s} /$ at higher steps 
Table S3. Evidence of effects on biass-f in Experiment 3. p>.1 not shown.

\begin{tabular}{|l|r|r|r|r|r|}
\hline Factor & \multicolumn{1}{|c|}{ B } & \multicolumn{1}{c|}{ SE } & \multicolumn{1}{c|}{ df } & \multicolumn{1}{c|}{ t } & \multicolumn{1}{c|}{ p } \\
\hline Frication & 0.305 & 0.015 & 15 & 19.83 & $<0.001$ \\
\hline Transition & 0.121 & 0.032 & 10 & 3.77 & 0.003 \\
\hline Rounding & 0.109 & 0.077 & 18 & 1.42 & \\
\hline Sentential transition & 0.009 & 0.009 & 8016 & 0.94 & \\
\hline Frication $\times$ transition & 0.003 & 0.010 & 15 & 0.32 & \\
\hline Frication $\times$ rounding & -0.053 & 0.031 & 15 & 1.72 & \\
\hline Frication $\times$ Sentential transition & -0.003 & 0.005 & 8021 & 0.32 & \\
\hline Transition $\times$ rounding & 0.061 & 0.064 & 10 & 0.95 & \\
\hline Transition $\times$ Sentential transition & -0.003 & 0.018 & 8022 & 0.33 & \\
\hline Rounding $\times$ Sentential transition & 0.006 & 0.018 & 8016 & 0.36 & \\
\hline Fric $\times$ trans $\times$ round & -0.016 & 0.020 & 15 & 0.81 & \\
\hline Fric $\times$ trans $\times$ sentential trans & -0.002 & 0.011 & 8014 & 0.18 & \\
\hline Fric $\times$ round $\times$ sentential trans & -0.010 & 0.011 & 8021 & 0.90 & \\
\hline Trans $\times$ round $\times$ sentential trans & 0.036 & 0.036 & 8022 & 1.00 & \\
\hline Fric $\times$ trans $\times$ round $\times$ sentential trans & 0.000 & 0.021 & 8014 & 0.00 & \\
\hline
\end{tabular}

and /s/ transitions. Unlike in Experiments 1 and 2, there was no main effect of rounding, though this was likely due to the fact that rounding was manipulated between subject (a strong numerical effect can be observed in the fact that the coefficient $(\mathrm{B}=.109)$ is nearly as large as that of transition $(\mathrm{B}=.121)$. There was no effect of sentential transition, a fact that contributed to our decision to drop this from the primary analyses.

\section{Experiment 6}

In Experiment 6, we again investigated the timing of the use of frication step and transition. However, in this experiment, frication spectrum used a five-step rather than six-step continuum. More importantly, we also manipulated fricative length (long and short) was added as a stimulus cue. This was not expected to affect fricative identification (so we predict null effects in this analysis), but was expected to affect timing. We also included a VOT $\times$ VL continuum in the filler trials.

Effects of Fricative Cues. To determine whether there was evidence of effects of each stimulus cue on biass-f., we used a similar analysis as used in Experiments 1-3b, with the addition of length as a fixed effect. We significantly increased fit by adding random slope of frication step on subject $\left(\chi^{2}(2)=221.21, p<0.001\right)$ and adding random slope of frication step on word pair $\left(\chi^{2}\right.$ $(2)=110.05, p<0.001)$. Adding random slope of transition and its interaction with step on subject did not increase fit $\left(\chi^{2}(7)=7.78, p=0.35\right)$ but adding random slope of transition and its interaction with step on word pair $\operatorname{did}\left(\chi^{2}(7)=30.23, p<0.001\right)$. More complex models failed to converge. Therefore, the final model included frication, transition and length as fixed effects, with random slope of frication on subject and random slopes of frication and transition on word pair (5).

$$
\text { Bias } \sim \text { Step * Transition * Length }+(\text { step } \mid \text { subject })+(\text { step * transition } \mid \text { subject })
$$




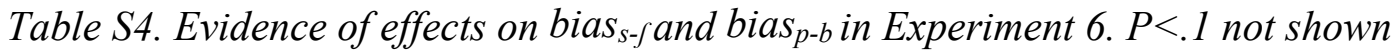

\begin{tabular}{|c|c|c|c|c|c|c|}
\hline & Factor & $\mathbf{B}$ & SE & df & $\mathbf{t}$ & p \\
\hline \multirow{15}{*}{$\begin{array}{c}\text { Fricatives } \\
\left(\text { bias }_{S-f)}\right.\end{array}$} & Step & 0.313 & 0.01 & 11 & 17.36 & $<.001$ \\
\hline & Transition & 0.097 & 0.02 & 6 & 3.89 & 0.008 \\
\hline & Rounding & 0.133 & 0.06 & 8 & 2.06 & 0.071 \\
\hline & Length & 0.019 & 0.00 & 3485 & 2.03 & 0.042 \\
\hline & Step $\times$ Transition & -0.002 & 0.00 & 9 & 0.25 & \\
\hline & Step $\times$ Rounding & 0.074 & 0.03 & 11 & 2.05 & 0.066 \\
\hline & Step $\times$ Length & -0.029 & 0.00 & 3485 & 4.40 & $<.001$ \\
\hline & Transition $\times$ Rounding & 0.062 & 0.05 & 6 & 1.24 & \\
\hline & Transition $\times$ Length & -0.011 & 0.01 & 3485 & 0.61 & \\
\hline & Rounding $\times$ Length & -0.002 & 0.01 & 3485 & 0.08 & \\
\hline & Step $\times$ Transition $\times$ Rounding & -0.022 & 0.01 & 9 & 1.40 & \\
\hline & Step $\times$ Transition $\times$ Length & -0.015 & 0.01 & 3485 & 1.17 & \\
\hline & Step $\times$ Rounding $\times$ Length & 0.002 & 0.01 & 3485 & 0.13 & \\
\hline & Transition $\times$ Rounding $\times$ Length & -0.019 & 0.03 & 3485 & 0.52 & \\
\hline & Step $\times$ Trans $\times$ Round $\times$ Length & -0.027 & 0.02 & 3485 & 1.03 & \\
\hline \multirow{3}{*}{$\begin{array}{c}\text { Stops } \\
\text { (biasp-b) }\end{array}$} & VOT & -0.37 & 0.03 & 4.60 & 13.64 & $<0.001$ \\
\hline & $\mathrm{VL}$ & 0.07 & 0.02 & 1702 & 4.41 & $<0.001$ \\
\hline & $\mathrm{VOT} \times \mathrm{VL}$ & 0.00 & 0.01 & 1702 & 0.28 & \\
\hline
\end{tabular}

Table S4 shows the results. There were significant main effects of frication $(\mathrm{p}<0.001)$ and transition $(\mathrm{p}=0.008)$ which were similar to those found in Experiments 1-3: more /s/ bias for high frication steps and for $/ \mathrm{s} /$ transitions. As expected there was also a main effect of rounding Somewhat surprisingly, there was a significant main effect of length $(p=0.04)$ with long fricatives showing more $/ \mathrm{s} /$ bias than short fricatives, and a length $\times$ frication interaction in which long fricatives showed greater bias towards /s/ at low steps but no bias at high steps. However, this was numerically small effect — at step 2 (where the effect was the largest), the difference in bias was only .067; in contrast the largest effect of transition (at step 3) was .20, and the largest effect of rounding (at step 4) was .37. Thus, the effect of length even if real is not learge.

Stop Consonant Voicing. For the filler (stop consonant) stimuli, Experiment 6 was similar to Experiment 2, except that it used a five-step continuum instead of six-step. Once again, we examined the effect of VOT and VL on biasp-busing a linear mixed-effects model. Our base model included both fixed effects and random intercepts for subject and word pair. Adding random slope of VOT on subject significantly increased fit $\left(\chi^{2}(2)=130.26, p<0.001\right)$. Adding random slope of VOT on word pair also significantly increased fit $\left(\chi^{2}(2)=49.17, p<0.001\right)$. More complex models failed to converge. Our final model included length and VOT as fixed effects and random slopes of VOT on subject and word pair (6)

$$
\text { Bias } \sim \text { VOT } * \text { VL }+(\text { VOT } \mid \text { Subject })+(\text { VOT } \mid \text { Word pair })
$$

Results are shown in Table S.4. As in Experiment 2, there were main effects of VOT $(p<.001)$ and VL $(p<.001)$, with more bias for short VOTs and long vowels.

\section{Discussion}


Results were in the expected directions (e.g., more looks to /s/ objects for /s/ transitions). This validates our auditory stimuli. More importantly, they document that each of the factors of interest affected fixations overall, a necessary precursor for our subsequently examination of the timing of these effects. 


\section{S4. Mouse-click responses as a function of VOT and VL for Experiment 6.}

Mouse-click responses were evaluated for each study to ensure that each cue had the expected effect on stimulus identification. In Experiment 6, we did not report the mouse click analysis of the stop consonant stimuli in the main text because the stop continua served a secondary goal to that of the fricative stimuli. They were similar to those in Experiment 2 and reported here.

\section{Results}

We examined mouse-click responses as a function of VOT and VL (Figure S4).

Participants reliably labeled stimuli at Step 1 as $/ \mathrm{p} /$ and at Step 6 as /b/ words, and at intermediate steps, there were more /p/ responses for short words. These were analyzed using a logistic effects model similar to Experiment 2. We started with a base model that included all of the fixed effects and random intercepts for subject and word pair. Adding random slopes of VOT on subject $\left(\chi^{2}(2)=\right.$ $1920.5, \mathrm{p}<.001)$ and on word pair $\left(\chi^{2}(2)=\right.$ $565.36, \mathrm{p}<.001)$ significantly increased fit. More complex models failed to converge. The model we used included VOT and VL as fixed effects and random slopes of VOT on word pair and subject (7)

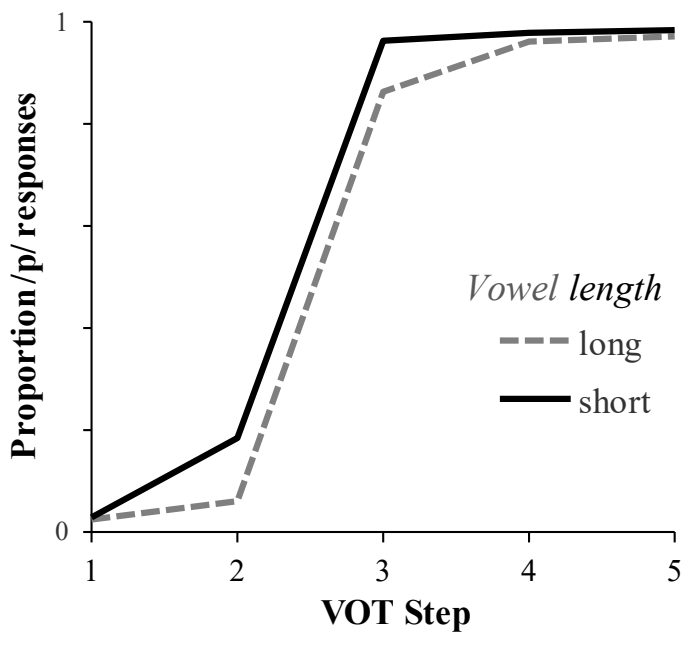

Figure S4. Experiment 6 mouse clicks to the $/ p /$ item as a function of VOT and VL.

$$
\mathrm{P} \text { response } \sim \mathrm{VOT} * \mathrm{VL}+(\mathrm{VOT} \mid \text { subject })+(\mathrm{VOT} \mid \text { word pair })
$$

Table S5 shows significant effects of VOT $(\mathrm{p}<.001)$, with more $/ \mathrm{p} /$ responses at higher steps, and VL $(\mathrm{p}<.001)$, with more $/ \mathrm{p} /$ responses for long vowels. The interaction was not significant.

\section{Discussion}

Results were in the expected direction. This validates the stimuli and also justifies further analyses as reported in the main text.
Table S5. Results of a mixed model examining mouse-click results for stop-consonant stimuli in Experiment 6.

\begin{tabular}{|l|r|r|r|c|}
\hline Factor & \multicolumn{1}{|c|}{ B } & \multicolumn{1}{c|}{ SE } & \multicolumn{1}{c|}{$\mathbf{z}$} & p \\
\hline VOT & 4.57 & 0.43 & 10.69 & $<.001$ \\
\hline VL & 1.17 & 0.06 & 19.17 & $<.001$ \\
\hline VOT x VL & 0.09 & 0.07 & 1.36 & \\
\hline
\end{tabular}




\section{S5. Timing of effects in eye-movement data for Experiment 6.}

Experiment 6 manipulated the length of the frication to ask whether the onset of the frication effect was timed to the onset of the vowel (or the end of the fricative). As part of this, we also assessed the timing of when specific phonetic cues were used: frication and transition for fricative stimuli, and VOT and VL for stop-consonant stimuli. These were replications of the findings of Experiments 1-3, and were not presented in the main text because their results were similar to those in Experiments 1-3. Here we present these analyses showing that the effect of frication was earlier than that of transition, as well as comparing the onset of the VOT and VL effect on the stop consonants.

\section{Results}

We estimated the onset of effects using the same techniques as Experiments 1-3. For trials with fricative stimuli, the biass-f. From we used the slope of the regression relating frication step to biass-f as our measure of the effect of frication step, and we used the difference in biass-f between the two transitions as our measure of transition. For stop-consonant stimuli, biasb-p was computed as before, and similar measures of effect size for both VOT and VL were computed from it. All effect sizes were computed at each 4 msec time step. Next, we normalized the timecourse of each effect by dividing by the maximum effect size, and jackknifed the data. Finally, we determined the onset of each effect by calculating when the jackknifed timecourse of each crossed and remained above a fixed threshold (e.g., the $20 \%$ threshold) for at least $30 \mathrm{msec}$. These timepoints were compared across cues with paired t-tests in which the t-statistic was adjusted to reflect the fact that each jackknifed participant represents N-1 participants.

Figure S5 and Table S6 show the onset of the effect of frication compared to the onset of the effect of transition. The effect of frication occurred marginally significantly earlier than the effect of transition at the $20 \%$ threshold. However, this difference was not significant at the $30 \%$ threshold, the $40 \%$ threshold, or the $50 \%$ threshold and was numerically later than the transition effect at the $40 \%$ and $50 \%$ thresholds. Moreover, even at the $20 \%$ threshold, where there was a marginal difference, both effects were quite late, and the difference was far shorter than the average of the fricative. Thus, this does not constitute strong evidence for an immediate activation account.

As seen in Figure S6 and Table S4, the effect of VOT occurred significantly earlier than the effect of VL using the $20 \%$ threshold. The difference between the onset of the VOT and VL

Table S6. Timing of fricative and stop-consonant effects in eye-tracking data for Experiment 6.

\begin{tabular}{|r|r|r|r|r|r|r|r|r|}
\hline \multirow{2}{*}{ Threshold } & \multicolumn{4}{|c|}{ Fricatives } & \multicolumn{3}{c|}{ Stop Consonant Voicing } \\
\cline { 2 - 10 } & \multicolumn{2}{|c|}{ Effect onset } & \multicolumn{2}{|c|}{$\begin{array}{c}\text { Fricative vs. } \\
\text { Trans }\end{array}$} & \multicolumn{2}{c|}{ Effect Onset } & \multicolumn{2}{c|}{ VOT vs. VL } \\
\cline { 2 - 10 } & $\begin{array}{c}\text { Fricative } \\
(\mathrm{ms})\end{array}$ & $\begin{array}{c}\text { Trans } \\
(\mathrm{ms})\end{array}$ & $\mathrm{t}(44)$ & $\mathrm{p}$ & $\begin{array}{c}\text { VOT } \\
(\mathrm{ms})\end{array}$ & VL (ms) & $\mathrm{t}(44)$ & $\mathrm{p}$ \\
\hline $20 \%$ & 654 & 717 & 2.00 & 0.05 & 499 & 574 & 2.49 & 0.02 \\
\hline $30 \%$ & 724 & 743 & 0.47 & & 546 & 599 & 0.73 & \\
\hline $40 \%$ & 792 & 767 & 0.74 & & 598 & 707 & 1.09 & \\
\hline $50 \%$ & 864 & 808 & 1.69 & & 660 & 758 & 1.69 & \\
\hline
\end{tabular}


effects was not significant at the $30 \%$ threshold, the $40 \%$ threshold, or the $50 \%$ threshold, although the onset of VOT effects was earlier than VL effects at all thresholds.

\section{Discussion}

As in Experiments 1-3, despite the fact that the fricative cue is available earlier than transition cues, the fricative cues were not used significantly earlier than transition cues. In fact, at the $40 \%$ and $50 \%$ thresholds, they were used after the transition cue (although this difference was not significant). Additionally, the stop-consonant results show cues being used in the expected order: VOT is presented earlier and used earlier than VL, though the evidence for this was not as robust as other experiments. This replication provides further support for buffered fricative processing.

A.

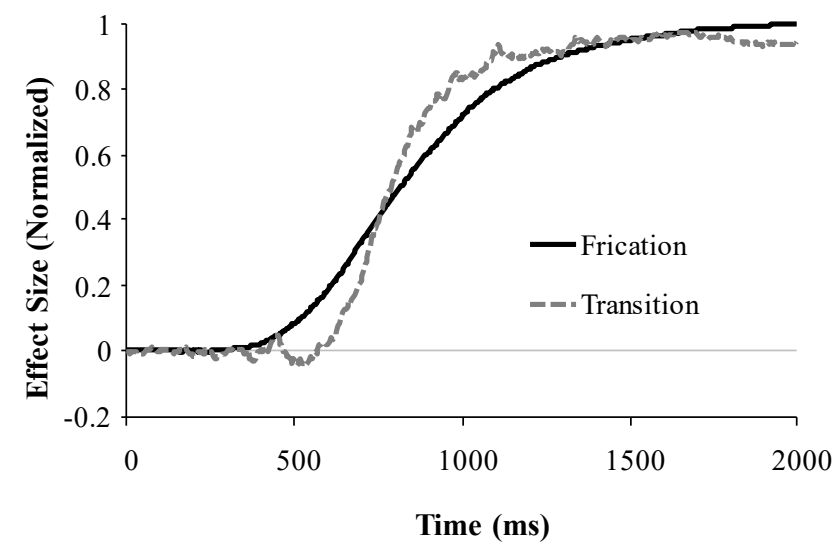

B.

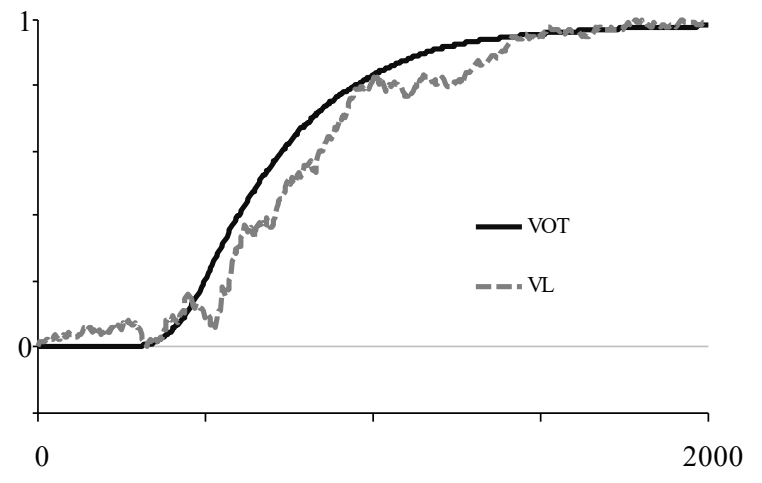

Figure S4. Normalized and jackknifed effect sizes as a function of time for A) for frication and transition and B) VOT and VL in Experiment 5. 


\section{S6. Detailed Methodological Comparison to Prior Work}

As we describe in the general discussion, the strong pattern of buffered processing conflicts with all other prior studies on asynchronous cue integration for voicing, manner and vowels (McMurray, Clayards, et al., 2008; Reinisch \& Sjerps, 2013; Toscano \& McMurray, $2012,2015)$. The fact that this has been shown now across five experiments suggests particularly strong evidence for this finding. However, these findings show discrepancies from several studies of fricatives. These likely rest on methodological differences.

Two gating studies (Smits, Warner, McQueen, \& Cutler, 2003; Warner, McQueen, \& Cutler, 2014) suggest that even at short gates, listeners are highly accurate at identifying fricatives. This is consistent with our own Experiment 4, but inconsistent with a memory buffer account. These results are not inconsistent with our VWP results: when forced to (i.e., no other information is forthcoming as in a gating task), listeners can make decisions on the basis of frication alone; however, in more natural uninterrupted speech they do not, and instead wait for the vocoid.

Kingston et al. (2016) shows indirect evidence for immediate integration for some fricatives. This study focused on the timing of the use of lexical status (e.g., the Ganong effect) relative to fricative onset. It examined a different fricative distinction (/s/ vs. /f/), but did not manipulate a secondary cue at vowel onset. This makes it difficult to draw strong conclusions about when frication is used relative to other cues. However, they show some influence of the information in the frication during the 300-400 msec timewindow (100-200 msec post fricative onset). This includes only the frication (fricatives were $150 \mathrm{msec}$ long). However, this effect was numerically much smaller than become at later points in processing, and it was only significant without correction for family-wise error $^{1}$ (see Supplement S2 for similar analyses with our own results). We are hesitant to take this as strong evidence for immediate integration (both due to the lack of a secondary cue, and the unclear statistical evidence).

If this effect is real, it would be consistent with the idea that frication can be used before the onset of the vowel, much earlier than was seen here. If true, four methodological differences could explain the mismatch. First, the dimension of sibilance vs. non-sibilance (/s/vs. /f/), may be more discriminable than place of articulation enabling immediate integration. McMurray and Jongman (2011), for example, show that listeners rarely make errors that cross sibilance classes. However, this explanation seems unlikely given that in our studies listeners do not even decide whether the sound was a fricative or a stop (an even easier distinction) until the vocoid.

Second, Kingston et al.'s (2016) sample averaged stimuli, may afford different sensory processing (c.f., Methods, Experiment 1) as the middle steps actually consist of both endpoints, superimposed. This may change the problem from categorizing ambiguous cue values to detecting one stimulus (in the presence of another). It is not clear whether this perceptual problem encourages immediate integration or not, but this difference may be important.

Third, Kingston et al. (2016) do not present the numerical amount of bias in the eyemovements at those early time-bins. As Supplement S2 shows, a statistically reliable effect at early times can be accompanied by an extremely small effect in real terms. There are hints of this in their own data: the $\mathrm{Z}$ statistic for the fricative effect at their $200 \mathrm{msec}$ bin is close to 2.0 at the first time bin, but it doesn't reach its ultimate strength of 10.0 until almost $1000 \mathrm{msec}$.

Finally, and most importantly, this study used only two alternatives, and the visual items

\footnotetext{
${ }^{1}$ Later, more statistically robust timewindows overlapped partially or fully with the vocoid and so do not pose a conflict with our results.
} 
were not pictures (which tap lexical level activation), but letters (to tap phoneme decisions). This was appropriate given the goals of their study; but in this case, it creates a situation perhaps analogous to our Experiment 2 that listeners can use information in the fricative if they are forced to (in our case by gated stimuli). Experiment 2 also used a 2AFC phoneme decision task, suggesting a strong similarity between this experiment and the Kingston et al. (2016) study. Letter choices may also tap a lower levels of processing (e.g., phonemes), supported by findings that listeners respond less gradiently (more categorically) with letters than pictures (McMurray, Aslin, Tanenhaus, Spivey, \& Subik, 2008). If this is the case one might conclude that prior to the vocoid the contents of the buffer are available to phonological processing, but not lexical.

It is not clear that the Kingston et al. (2016) study offers unambiguous evidence for immediate integration (and this was not their goal), and they did not examine multiple cues. However, even if their results are treated as evidence for immediate use of frication, our use of a lexical level task, a richer response set, and more realistically synthesized stimuli suggests that our results may be more representative of natural language processing.

The final study that should be considered is that of (Mitterer \& Reinisch, 2013). They also examined the /s/ vs. /f/ distinction, but in word-final position. They appear to find rapid use of the frication as well. The best contrast with our own work is Experiment 3, in which fricatives appeared in the context of carrier sentences (in medial position). This experiment still observed delayed use of frication that was coincident with the use of other cues. There may be a number of differences that are relevant.

First we point out that the t-statistic in their "immediate" bin (200-300 msec) was barely above 2.0, and may not have survived multiple comparison testing (which they did not do).

Second, like Kinston et al, they use a long time bin for their analysis $(100 \mathrm{msec})$. However, in their study it was not clear how long their fricatives were (this was not reported in the text). Thus, results in this bin could reflect processing most of the way through a short(ish) fricative. As a result, the evidence for immediate use of frication cues in this study is not strong, even if we take their results at face value. Third, the text-based of the VWP (a version of which we note was also used by Kingston) may put undue focus on the sound rather than the lexical identity of the word (McMurray, Aslin, et al., 2008), and it may heighten competition effects, since similar sounding words also look similar (Dahan \& Tanenhaus, 2005).

However, most importantly, in word-final position there is literally no other information that will arrive after the fricative. In that case, there is no need to wait -there is nothing to integrate with the fricative. In fact, the vowel (and the formant transitions) has already arisen. This then may support the hypothesis that the vowel that is necessary for initiating lexical access (see the general discussion). In our case, even in a sentence context, the vowel for the target word does not arrive until after the fricative (delaying lexical access), while in Mitterer and Reinisch (2013) the vowel would have arrived (and lexical access begun) by the time the fricative was heard.

\section{References}

Dahan, D., \& Tanenhaus, M.K. (2005). Looking at the rope when looking for the snake: Conceptually mediated eye movements during spoken-word recognition. Psychonomic Bulletin \& Review, $12(3), 453-459$.

Kingston, J., Levy, J., Rysling, A., \& Staub, A. (2016). Eye Movement Evidence for an Immediate Ganong Effect. Journal of Experimental Psychology: Human Perception and Performance, 42(12), 1969-1988. 
McMurray, B., Aslin, R.N., Tanenhaus, M.K., Spivey, M.J., \& Subik, D. (2008). Gradient sensitivity to within-category variation in words and syllables. Journal of Experimental Psychology, Human Perception and Performance, 34(6), 1609-1631.

McMurray, B., Clayards, M., Tanenhaus, M.K., \& Aslin, R.N. (2008). Tracking the time course of phonetic cue integration during spoken word recognition. Psychonomic Bulletin and Review, 15(6), 1064-1071.

McMurray, B., \& Jongman, A. (2011). What information is necessary for speech categorization? Harnessing variability in the speech signal by integrating cues computed relative to expectations. Psychological Review, 118(2), 219-246.

Mitterer, H., \& Reinisch, E. (2013). No delays in application of perceptual learning in speech recognition: Evidence from eye tracking. Journal of Memory and Language, 69(4), 527-545.

Reinisch, E., \& Sjerps, M.J. (2013). The uptake of spectral and temporal cues in vowel perception is rapidly influenced by context. Journal of Phonetics, 41(2), 101-116.

Smits, R., Warner, N., McQueen, J.M., \& Cutler, A. (2003). Unfolding of phonetic information over time: a database of Dutch diphone perception. J Acoust Soc Am, 113(1), 563-574.

Toscano, J.C., \& McMurray, B. (2012). Online integration of acoustic cues to voicing: Natural vs. synthetic speech. Attention, Perception \& Psychophysics, 74(6), 1284-1301.

Toscano, J.C., \& McMurray, B. (2015). The time-course of speaking rate compensation: Effects of sentential rate and vowel length on voicing judgments. Language, Cognition and Neuroscience, 30, 529-543.

Warner, N., McQueen, J.M., \& Cutler, A. (2014). Tracking perception of the sounds of English. J Acoust Soc Am, 135(5), 2995-3006. 